Prepared in cooperation with the Kansas Water Office

\title{
Methods for Estimating Low-Flow Frequency Statistics, Mean Monthly and Annual Flow, and Flow-Duration Curves for Ungaged Locations in Kansas
}

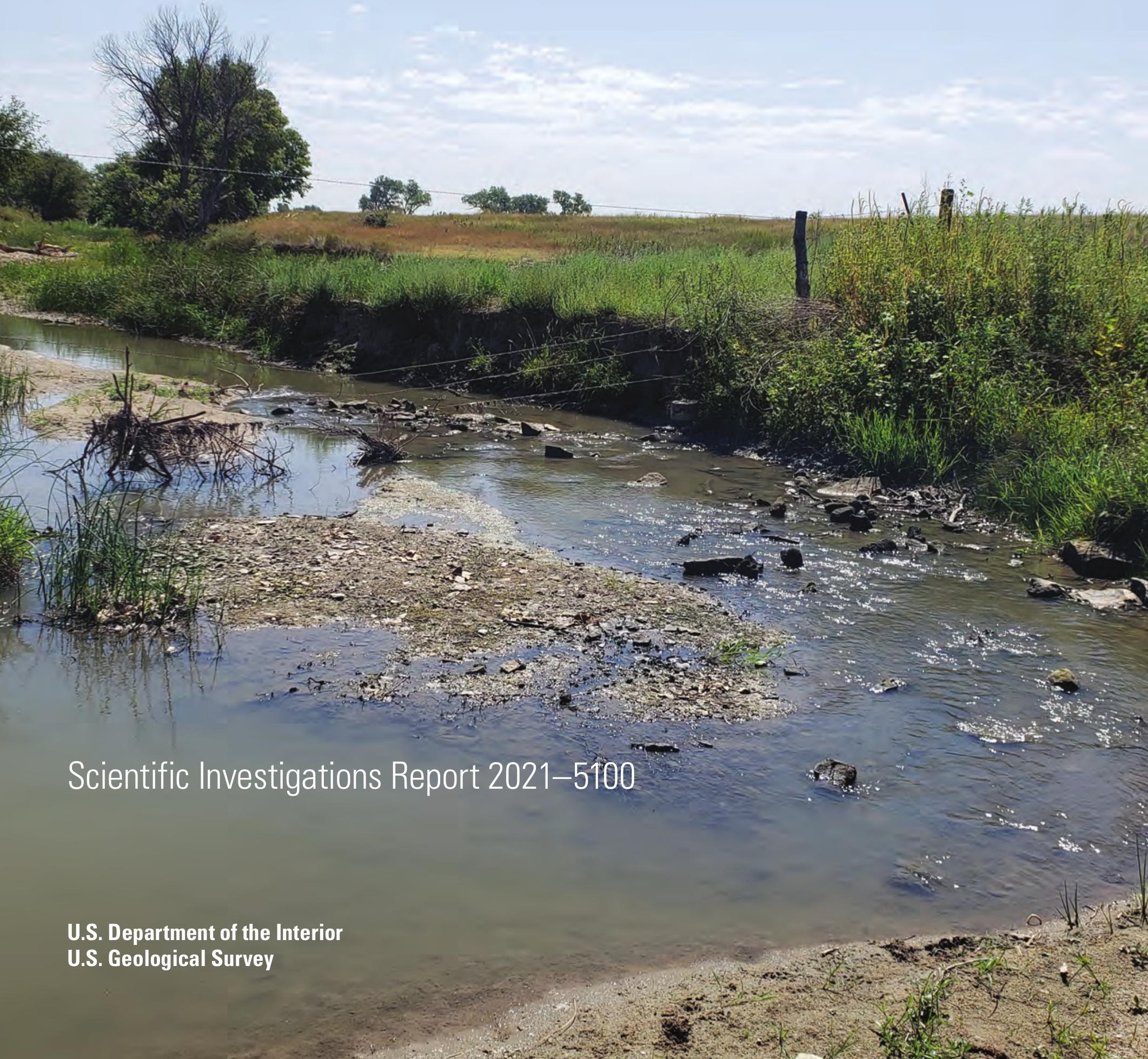


Cover: Photograph showing 1.02 cubic feet per second $\left(\mathrm{ft}^{3} / \mathrm{s}\right)$ over riffle control at Saline River near Wakeeny, Kansas (U.S. Geological Survey [USGS] station 06866900), taken August 25, 2021, by Tyler Saryerwinnie, USGS.

Back cover: Left: Photograph showing $0.64 \mathrm{ft}^{3} / \mathrm{s}$ over riffle control at Kill Creek at 95 th Street near De Soto, Kans. (USGS station 06892360), taken September 21, 2018, by Sami Milk, USGS.

Top, right: Photograph showing $0.85 \mathrm{ft}^{3} / \mathrm{s}$ over riffle control at Stranger Creek at Easton, Kans. (USGS station 06891850), taken October 1, 2018, by Bradley Lukasz, USGS.

Bottom right: Photograph showing observed zero flow at South Fork Republican River near Colorado-Kansas State Line (USGS station 06827000), taken September 7, 2021, by Lori Marintzer, USGS. 


\section{Methods for Estimating Low-Flow \\ Frequency Statistics, Mean Monthly and Annual Flow, and Flow-Duration Curves for Ungaged Locations in Kansas}

By Bradley S. Lukasz

Prepared in cooperation with the Kansas Water Office

Scientific Investigations Report 2021-5100 


\section{U.S. Geological Survey, Reston, Virginia: 2021}

For more information on the USGS - the Federal source for science about the Earth, its natural and living resources, natural hazards, and the environment—visit https://www.usgs.gov or call 1-888-ASK-USGS.

For an overview of USGS information products, including maps, imagery, and publications, visit https://store.usgs.gov/.

Any use of trade, firm, or product names is for descriptive purposes only and does not imply endorsement by the U.S. Government.

Although this information product, for the most part, is in the public domain, it also may contain copyrighted materials as noted in the text. Permission to reproduce copyrighted items must be secured from the copyright owner.

Suggested citation:

Lukasz, B.S., 2021, Methods for estimating low-flow frequency statistics, mean monthly and annual flow, and flow-duration curves for ungaged locations in Kansas: U.S. Geological Survey Scientific Investigations Report 2021-5100, 69 p., https://doi.org/10.3133/sir20215100.

Associated data for this publication:

U.S. Geological Survey, 2018, USGS water data for the Nation: U.S. Geological Survey National Water Information System database, https://doi.org/10.5066/F7P55KJN.

ISSN 2328-0328 (online) 


\section{Contents}

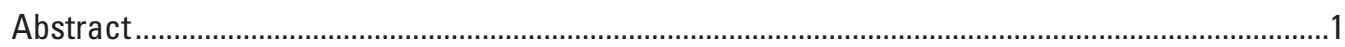

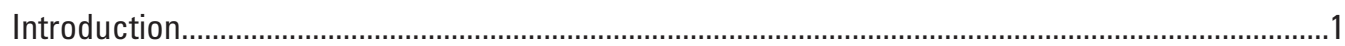

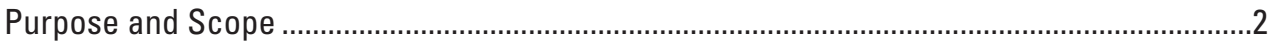

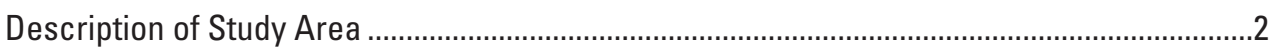

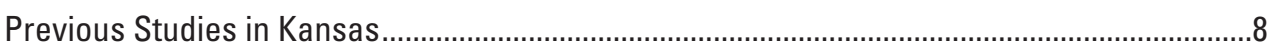

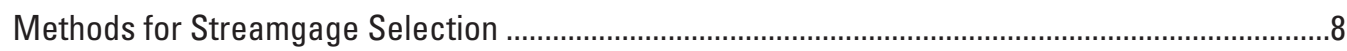

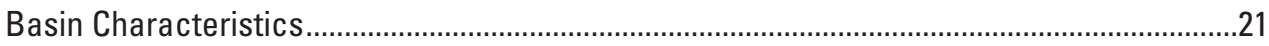

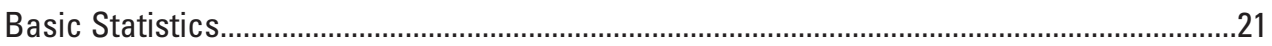

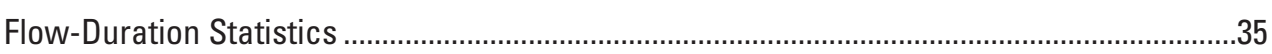

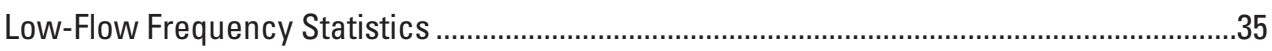

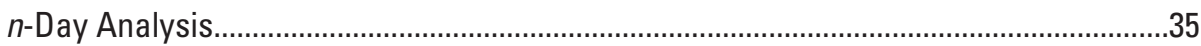

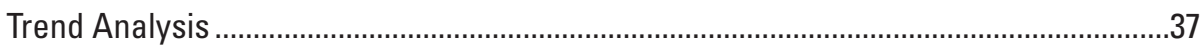

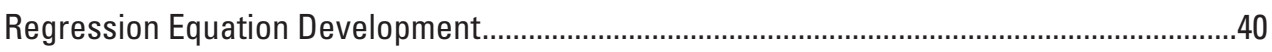

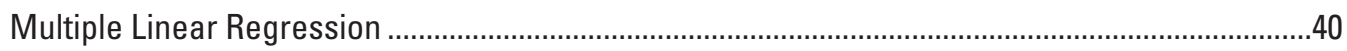

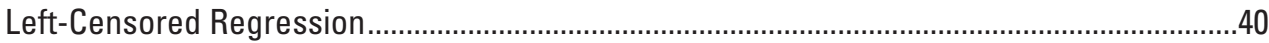

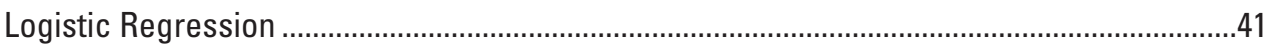

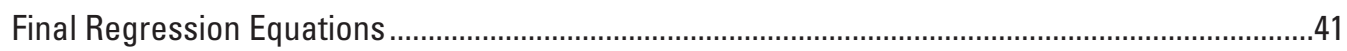

Accuracy and Limitations of Regression Equations ........................................................55

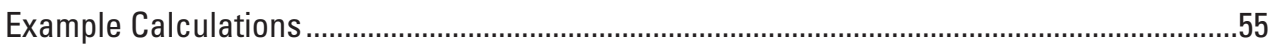

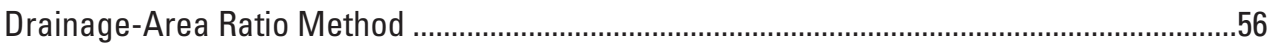

Comparison to Regression Model.......................................................................................

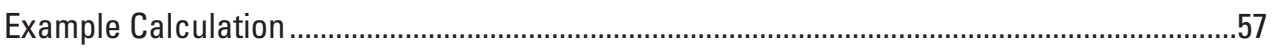

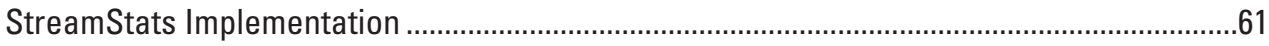

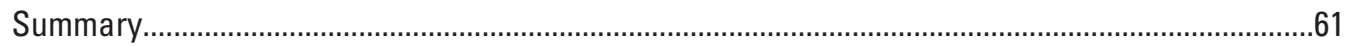

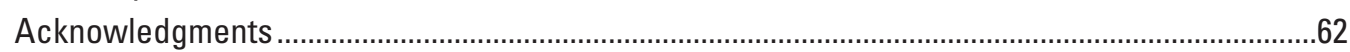

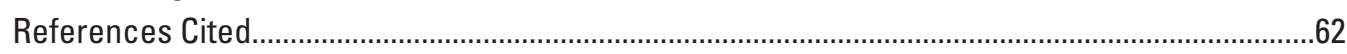

Appendix 1. Flow-Duration Curve Information .......................................................................67

Appendix 2. Low-Flow Frequency Statistics Information .............................................................68

Appendix 3. Basin Characteristic Information ........................................................................69

\section{Figures}

1. Map showing locations of U.S. Geological Survey streamgages in Kansas and parts of surrounding States that were used to develop regression equations...................3

2. Map showing land-surface elevation in Kansas and parts of surrounding States...........4

3. Map showing land-surface slope in Kansas and parts of surrounding States..................5

4. Map showing land use in Kansas and parts of surrounding States with annual minimum 7-day streamflow trends

5. Map showing distribution of mean annual precipitation (1981-2010) in Kansas and parts of surrounding States and the hydrologic region boundary used in this study

6. Map showing active Kansas River U.S. Geological Survey streamgages and

U.S. Army Corps of Engineers reservoirs that regulate the flow in the Kansas River ....20 
7. Graph showing flow-duration curve for the U.S. Geological Survey streamgage Kansas River at De Soto, Kansas.

8. Graph showing annual minimum 7-day mean streamflow and calculated 7010 at U.S. Geological Survey streamgage Little Blue River near Barnes, Kansas.. .36

9. Graphs showing selected periods of record for U.S. Geological Survey streamgage Little Blue River near Barnes, Kansas, for which a Mann-Kendall test for detection of trends was assessed.

10. Map showing annual mean temperature and annual mean precipitation trends by National Oceanic and Atmospheric Administration climate divisions within the study area, 1987-2017, and annual minimum 7-day mean flow with 10-year recurrence interval streamflow trends at streamgages for their period of record through 2017

\section{Tables}

1. Active and inactive U.S. Geological Survey streamgages used for regression analysis within the study area

2. Active and inactive U.S. Geological Survey streamgages used for regression analysis within the study area

3. Active and inactive U.S. Geological Survey streamgages used for regression analysis within the study area

4. List of active U.S. Geological Survey streamgages on the Kansas and Arkansas Rivers

5. Annual low-flow frequency statistics for active U.S. Geological Survey streamgages on the Kansas and Arkansas Rivers ....

6. Basin characteristics tested for significance during the regression analysis

7. Basic statistics for daily mean streamflows of U.S. Geological Survey streamgages used in the regression analysis, computed using SWToolbox

8. Basic statistics for daily mean streamflows of U.S. Geological Survey streamgages used in the regression analysis, computed using SWToolbox

9. Regression equations for estimating selected low-flow frequency statistics for unregulated streams in the east region of Kansas.....

10. Regression equations for estimating selected low-flow frequency statistics for unregulated streams in the west region of Kansas

11. Pairs of U.S. Geological Survey streamgages for drainage-area ratio versus regional regression equations analysis.

12. Medians and standard deviations of the absolute differences between annual mean 7-day low flow with a recurrence interval of 10 years using observed streamflow, the drainage-area ratio method, and regional regression equations 


\section{Conversion Factors}

U.S. customary units to International System of Units

\begin{tabular}{lcl}
\hline & \multicolumn{1}{c}{ Multiply } & \multicolumn{1}{c}{ To obtain } \\
\hline & Length & \\
\hline inch (in.) & 2.54 & centimeter $(\mathrm{cm})$ \\
inch (in.) & 25.4 & millimeter $(\mathrm{mm})$ \\
foot (ft) & 0.3048 & meter $(\mathrm{m})$ \\
mile (mi) & 1.609 & kilometer $(\mathrm{km})$ \\
\hline & Area & \\
\hline square mile $\left(\mathrm{mi}^{2}\right)$ & 259.0 & hectare $(\mathrm{ha})$ \\
square mile $\left(\mathrm{mi}^{2}\right)$ & 2.590 & square kilometer $\left(\mathrm{km}^{2}\right)$ \\
\hline & Flow rate & \\
\hline cubic foot per second $(\mathrm{ft} 3 / \mathrm{s})$ & 0.02832 & cubic meter per second $\left(\mathrm{m}^{3} / \mathrm{s}\right)$ \\
\hline
\end{tabular}

\section{Datum}

Vertical coordinate information is referenced to the North American Vertical Datum of 1988 (NAVD 88).

Horizontal coordinate information is referenced to the North American Datum of 1983 (NAD 83). Elevation, as used in this report, refers to distance above the vertical datum.

\section{Supplemental Information}

A water year begins on 0ctober 1 of the previous calendar year and ends on September 30 of the year of interest; thus, the water year ending September 30, 2011, is called the "2011 water year."

A climatic year begins on April 1 and ends on March 31 of the following year. A climatic year is designated by the calendar year during which most of the 12 months occur. 


\section{Abbreviations}

$\begin{array}{ll}\text { BFI } & \text { base-flow index } \\ \text { CONTDA } & \text { contributing drainage area } \\ \text { DAR } & \text { drainage-area ratio } \\ \text { GIS } & \text { geographic information system } \\ \text { MLE } & \text { maximum-likelihood estimation } \\ \text { NWIS } & \text { National Water Information System } \\ p & \text { probability } \\ \text { PRECIPfull } & \text { mean annual precipitation for 1895-2017 } \\ \text { RMSE } & \text { root mean square error } \\ \text { SE } & \text { standard error } \\ \text { USGS } & \text { U.S. Geological Survey } \\ 705 & \text { annual minimum 7-day mean low flow with 5-year recurrence interval } \\ 7010 & \text { annual minimum 7-day mean flow with 10-year recurrence interval }\end{array}$




\title{
Methods for Estimating Low-Flow Frequency Statistics, Mean Monthly and Annual Flow, and Flow-Duration Curves for Ungaged Locations in Kansas
}

\author{
By Bradley S. Lukasz
}

\section{Abstract}

Knowledge of the magnitude, frequency, and duration of low flows is critical for water-supply management; reservoir design; waste-load allocation; and the preservation of water quality and quantity for irrigation, recreation, and ecological conservation purposes. The U.S. Geological Survey (USGS), in cooperation with the Kansas Water Office, completed a statewide study to develop regression equations for selected low-flow frequency and flow-duration statistics for ungaged streams in Kansas.

The low-flow statistics included the annual and monthly 1-, 7-, and 30-day mean low flow for a recurrence interval of 10 years; flow-duration exceedance probabilities of 0.01 , $0.1,2,5,10,25,50,75,90,95,99,99.9$, and 99.99 percent; and mean annual flow. Data used in this analysis were from 254 USGS continuous-record streamgages using data through March 31, 2017, for the regression equation analysis and using data through September 30, 2017, for the flow-duration curve analysis. The streamgages used in the regression analysis of this report are in Kansas and 50 miles beyond the borders of the State. A trend analysis was done because trends can introduce bias into results. Some trends were detected; however, no streamgage was omitted from the analysis because of the presence of a trend. Geographic-information-system software was used to compute 13 basin characteristics for each streamgage.

The State of Kansas was divided into two study areas for the regional regression analysis. Logistic and left-censored regression techniques were used to develop the equations because of the presence of zero flows in the datasets. A collection of performance metrics is provided to estimate the accuracy of each equation. These equations are only applicable to streams in Kansas that are not substantially affected by diversion, regulation, or urbanization. Basin characteristics of these ungaged locations also need to be within the range of the basin characteristics used to develop these equations.

The drainage-area ratio (DAR) method was tested against the regional regression equations using 19 pairs of streamgages. The 7-day annual mean low-flow statistic was estimated using the regression equations and the DAR method. The absolute difference, in percent, was calculated using the observed 7-day annual mean low flow for both methods. The results of the Wilcoxon signed-rank test indicated that the difference between the absolute differences, in percent, for the groups tested was not statistically significant. Previous USGS studies state it is preferable to use the DAR method when the ratio is between 0.5 and 1.5. Some studies offer no scientific basis for these guidelines, whereas other studies have developed different guidelines. In Kansas, the DAR method produced the smallest absolute difference, in percent, when the ratio of drainage areas was between 0.5 and 1.5. The results of this study indicate that the DAR method is appropriate for use in Kansas when estimating streamflow at an ungaged location if there is a nearby streamgage on the same river or stream where the DAR is between 0.5 and 1.5 and the low-flow statistic at the nearby streamgage is not zero.

The regression equations developed in this report will be incorporated into the USGS StreamStats web-based geographic-information-system tool. This will allow users to click on any ungaged location within StreamStats and compute estimates of the selected low-flow frequency and flowduration statistics. The low-flow frequency and flow-duration statistics for streamgages in Kansas also will be available in StreamStats.

\section{Introduction}

Knowledge of the magnitude, frequency, and duration of low flows is critical for water-supply management; reservoir design; waste-load allocation; and the preservation of water quality and quantity for irrigation, recreation, and ecological conservation purposes. Flow-duration curves and low-flow statistics, including $n$-day flow frequency, are used by Federal, State, and local entities for setting regulatory standards and establishing water-quality and water-supply management goals, particularly during dry periods when demand often exceeds the supply of water (Ries, 2006). Historically, the Kansas Department of Health and Environment has used the annual minimum 7-day mean streamflow with a 10 -year recurrence interval statistic (often referred to as "7Q10") to determine critical flow levels to maintain established 
water-quality criteria for Kansas streams. The U.S. Geological Survey (USGS) operates a monitoring network of streamgages in Kansas and throughout the Nation where water-resources information, collected in near-real time, is used for computing long-term (30 or more years) low-flow statistics and developing flow-duration curves. However, the USGS cannot operate streamgages at every location, so methods are needed to estimate low-flow frequency statistics and flow-duration curves at ungaged stream locations (Eash and Barnes, 2017; Gotvald, 2017; Painter and others, 2017).

Determination of low-flow statistics, mean annual and monthly flows, and flow durations for ungaged locations, using various statistical methods, is often needed to provide the necessary information to effectively manage water resources throughout the State. Thus, the USGS, in cooperation with the Kansas Water Office, completed a statewide study to develop regression equations for selected low-flow frequency and flow-duration statistics for ungaged streams in Kansas. Regionalization, a commonly used regression technique, includes the development of regression equations for a hydrologic region to estimate flow statistics at ungaged locations based on the relation of computed statistics at gaged locations to various physical and climate characteristics of that region. Alternative methods, including the drainage-area ratio (DAR) method, have been used to estimate flow statistics at ungaged locations on gaged streams for regulated and unregulated flows (Perry and others, 2004a). Other uses for low-flow frequency statistics are establishing minimum flow requirements for streams and rivers, permitting for wastewater discharges (Gotvald, 2017),making management and design decisions for hydroelectric facilities, fish passage (WilliamsSether and Gross, 2016), irrigation; recreation, and wildlife conservation (Ziegeweid and others, 2015).

\section{Purpose and Scope}

The purpose of this report is to present the results of the study to update low-flow frequency statistics and flowduration curves for gaged locations in Kansas with data available through 2017 and regression equations used to estimate low-flow frequency statistics, mean annual flow, and flowduration curves for ungaged locations in Kansas.

Regression equations will be incorporated into the StreamStats program, a web-based tool developed by the USGS (Ries and others, 2017) to automatically compute various streamflow statistics at user-specified locations on Kansas streams. The scope of this report includes stream locations that are not substantially affected by regulation, diversion, or urbanization. Streamflow statistics at streamgages affected by the previously mentioned activities were computed for completeness. Nationally, the methods and results presented in this report offer guidance and perspective for future studies concerned with estimating low-flow statistics at ungaged locations.

\section{Description of Study Area}

The study area (fig. 1) consists of the State of Kansas and neighboring areas within a 50-mile (mi) buffer of Kansas in the bordering States of Colorado, Missouri, Nebraska, and Oklahoma. The physical and climatic characteristics differ substantially across the study area, which results in a varied hydrologic streamflow response (Painter and others, 2017). Rivers, streams, and creeks in Kansas generally flow from west to east, following the topography of the landscape (Painter and others, 2017).

Land-surface elevations within Kansas range from about 700 feet (ft) above the North American Vertical Datum of 1988 at the Oklahoma State line in southeastern Kansas to about 4,135 ft above the North American Vertical Datum of 1988 near the Colorado border in western Kansas - a difference of about 3,435 ft (Rasmussen and Perry, 2000; Perry and others, 2004a, b; U.S. Geological Survey, 2019; fig. 2). Mean basin slope for the 254 streamgages in Kansas and the surrounding States used in the regression analysis is about 3.8 percent (fig. 3; U.S. Geological Survey, 2010).

Land use in Kansas is mainly agricultural, with pasture plus grassland (48.9 percent) and cropland (43.2 percent) accounting for much of the land use in the State (Fry and others, 2011; Painter and others, 2017; fig. 4). Forest cover and urban land use account for about 3.8 and 1.5 percent of the State, respectively (Fry and others, 2011; Painter and others, 2017).

A large precipitation gradient, from west to east, is typical in Kansas, as well as other regions in the central United States (Goodin and others, 2004; Painter and others, 2017). This mean annual precipitation gradient in Kansas is shown in figure 5. Mean annual precipitation ranges from about 18 inches (in.) in the far west to more than 44 in. in the southeastern corner of the State. The Rocky Mountains (not shown) cause a rain shadow effect, which can yield semidry to dry conditions in western Kansas, whereas moist air from the Gulf of Mexico, predominantly in the spring and summer months, produces more precipitation in the eastern part of the State (Goodin and others, 2004; Painter and others, 2017). Irrigation associated with groundwater withdrawals is common in the area west of the 28-in. precipitation contour line (fig. 5), which approximately splits the State in half (Painter and others, 2017). These withdrawals are substantial and are likely responsible for reduced flow conditions in the western part of Kansas (Juracek and Eng, 2017).

The west-to-east gradient in elevation and precipitation characteristics across the State also results in a west-to-east increase in mean annual surface-water runoff across Kansas (Painter and others, 2017). Mean annual runoff ranges from about 0.2 in. near the western Kansas border to around 8 in. near the eastern Kansas border (Gebert and others, 1987; Painter and others, 2017). Flows at streamgages in central and eastern Kansas have a typical temporal hydrologic distribution 


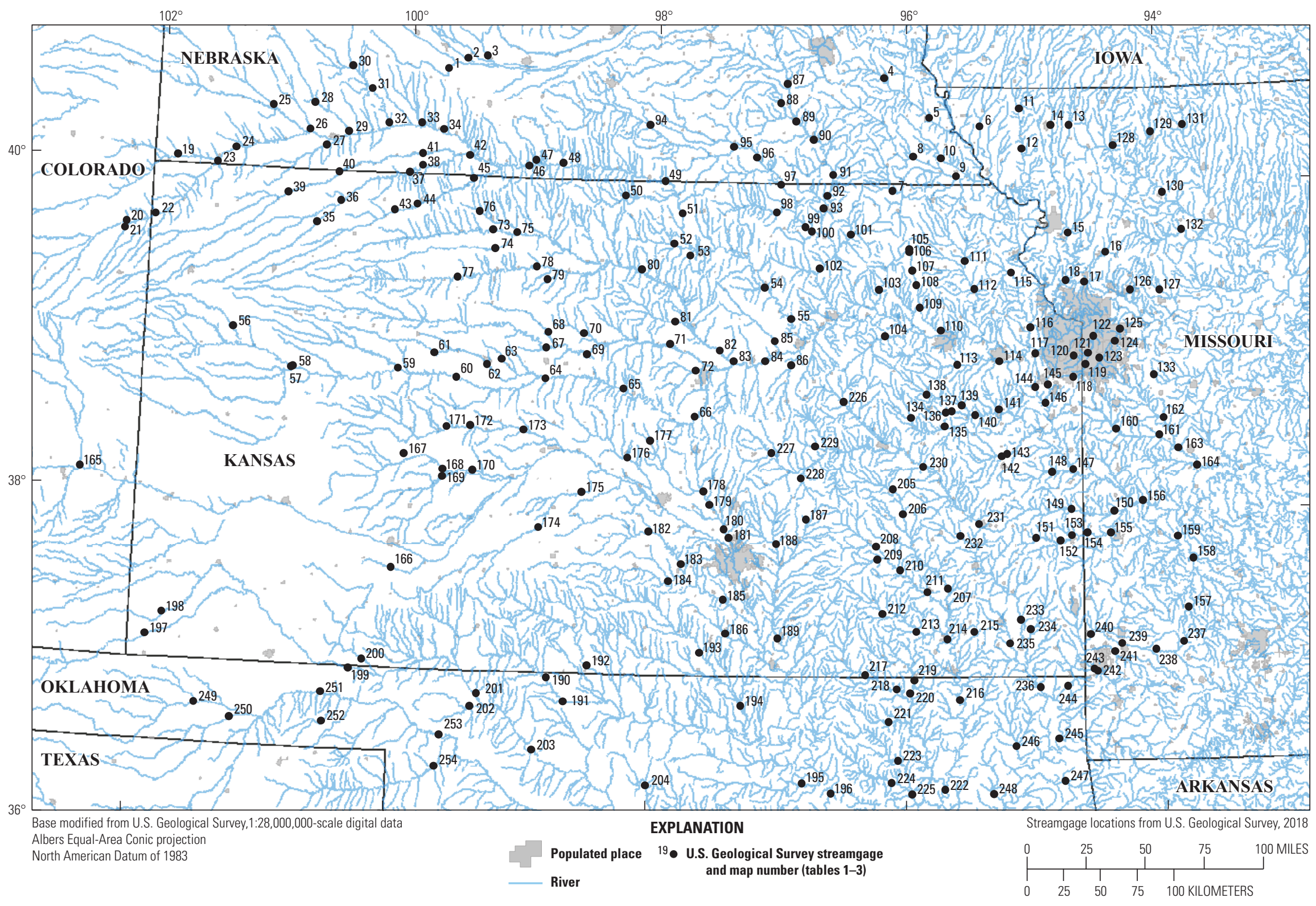

Figure 1. Locations of U.S. Geological Survey streamgages in Kansas and parts of surrounding States that were used to develop regression equations. 


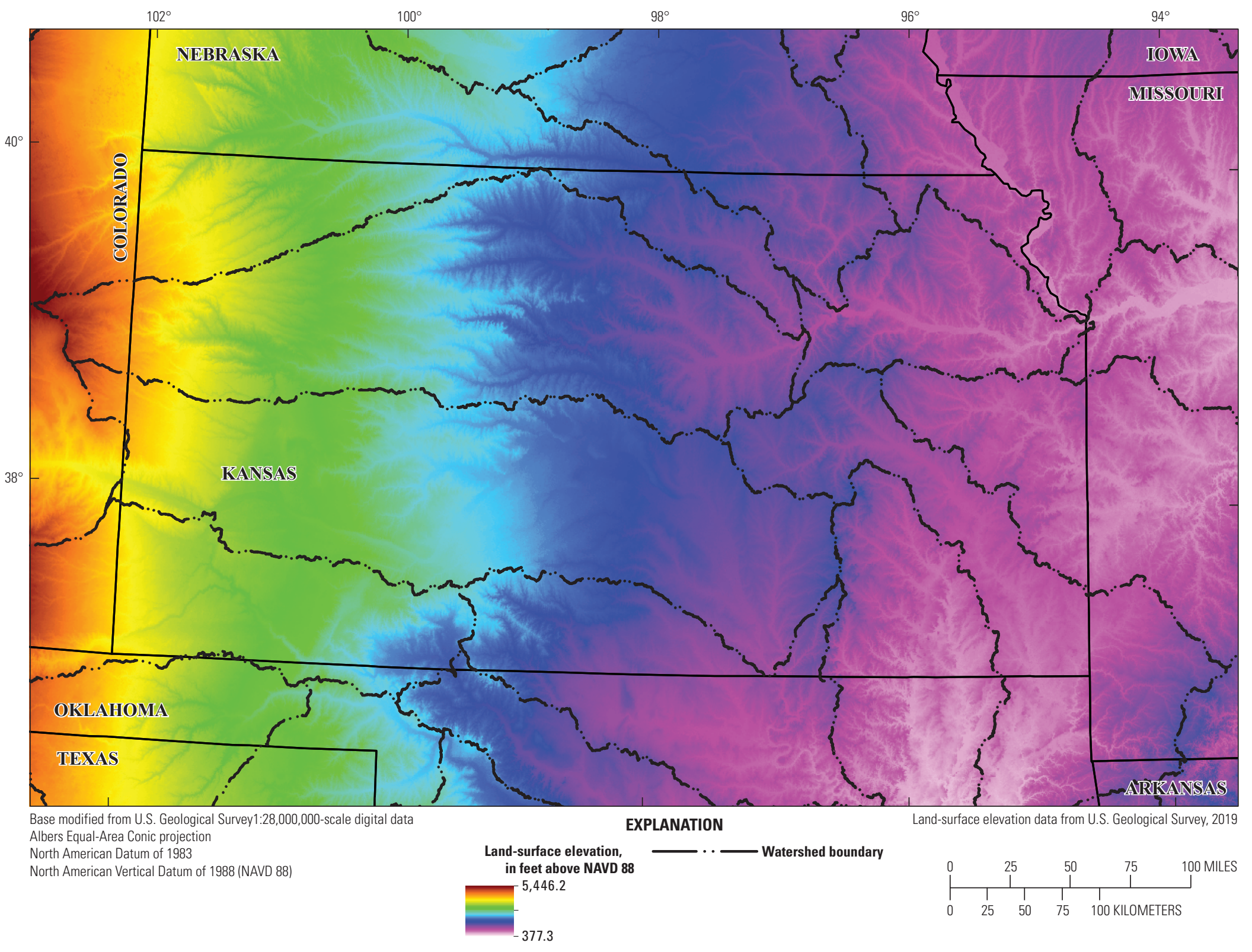

Figure 2. Land-surface elevation in Kansas and parts of surrounding States. 


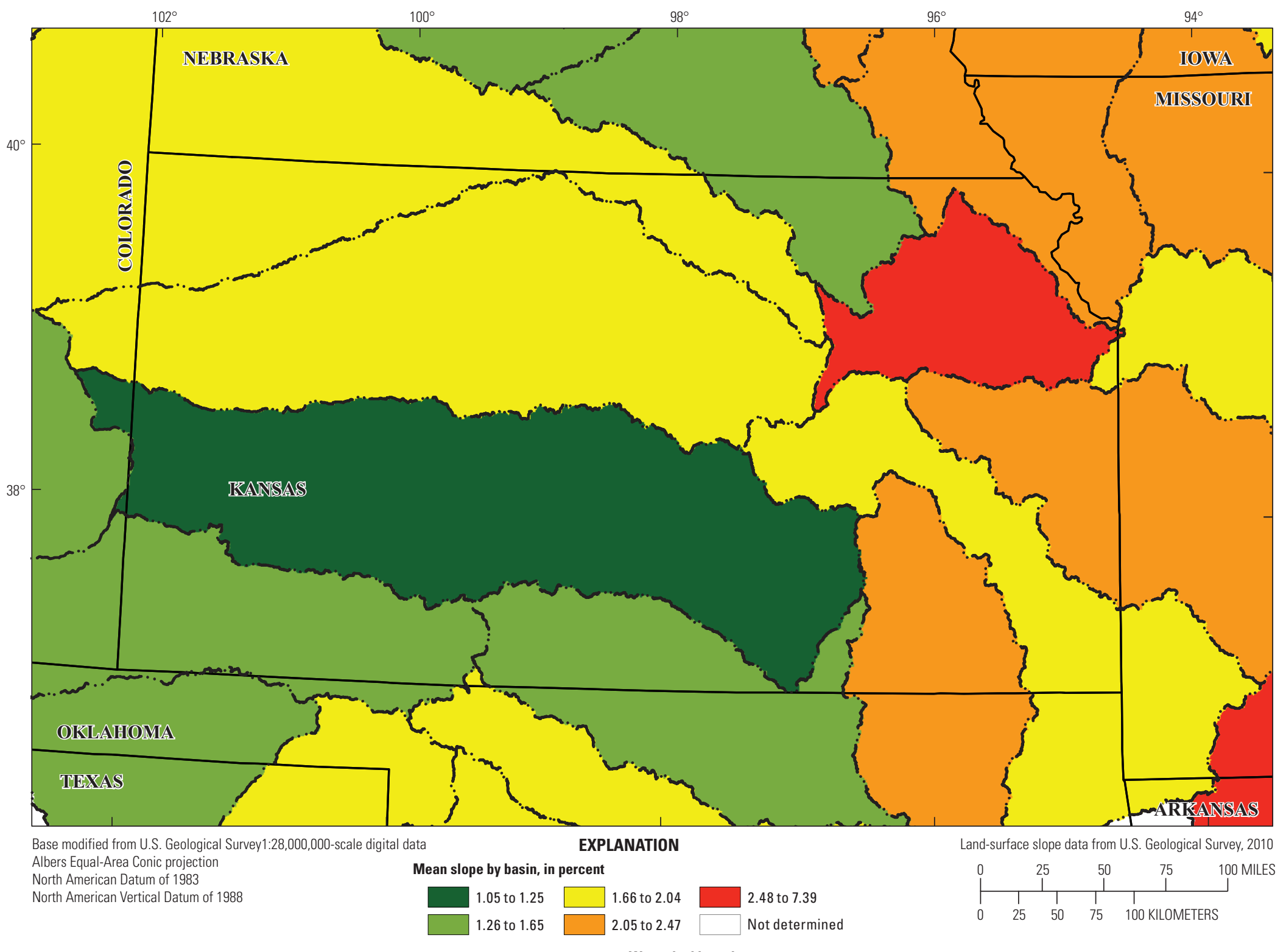

— - W Watershed boundary

Figure 3. Land-surface slope in Kansas and parts of surrounding States. 


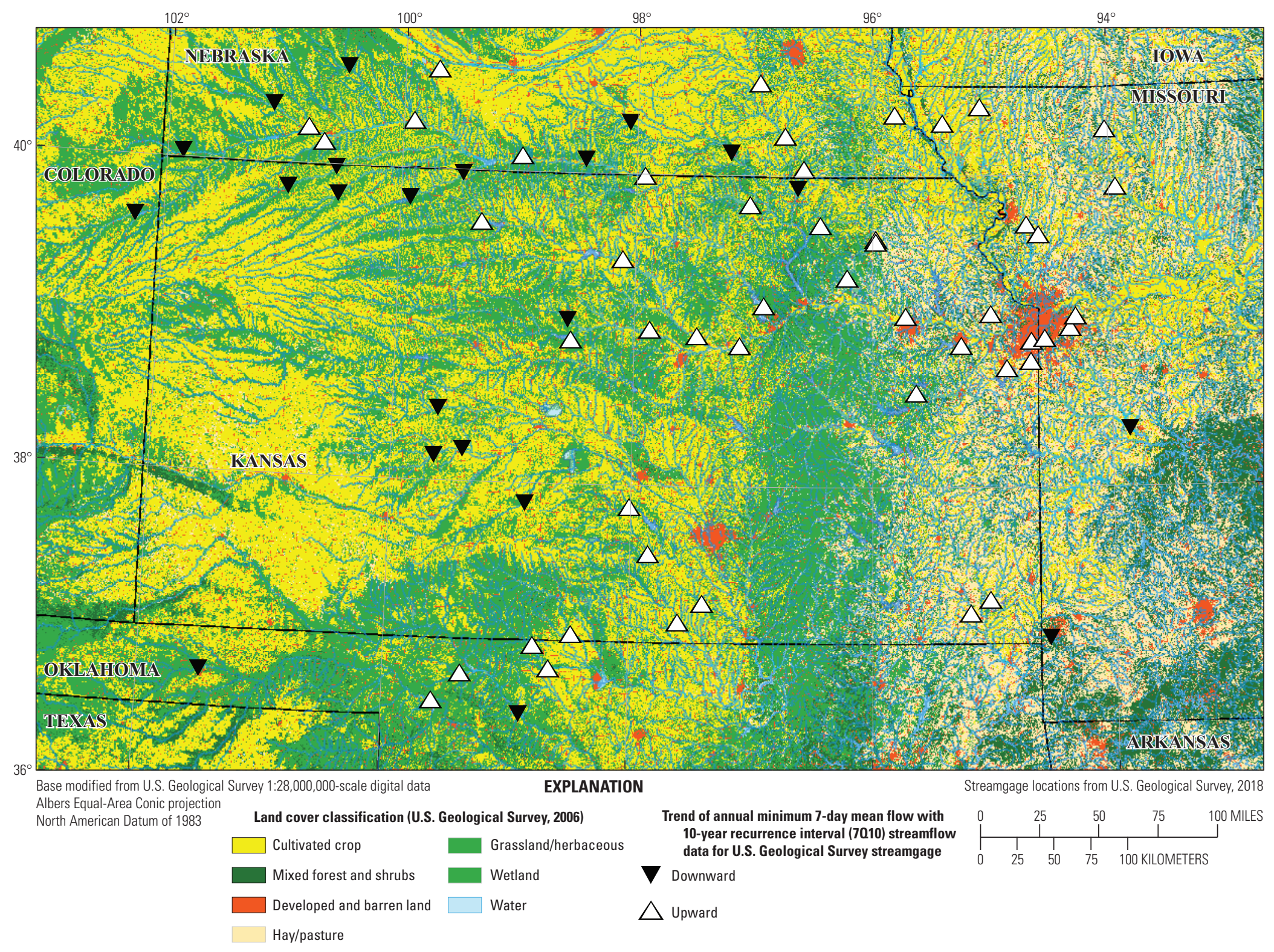

Figure 4. Land use in Kansas and parts of surrounding States with annual minimum 7-day streamflow trends. 


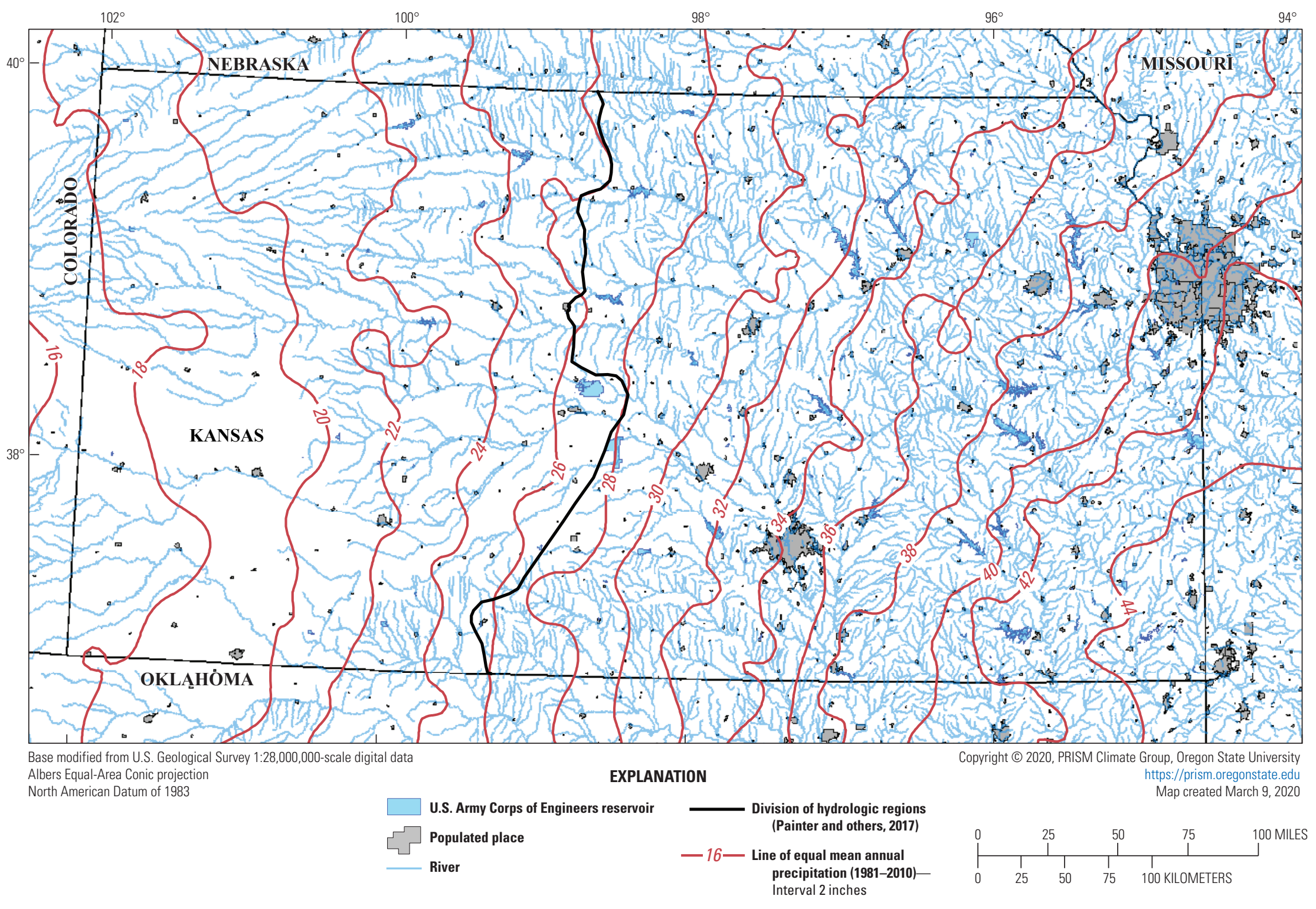

Figure 5. Distribution of mean annual precipitation (1981-2010) in Kansas and parts of surrounding States and the hydrologic region boundary used in this study. 
with greater mean monthly streamflows in the spring and early summer months (Moody and others, 1986; Painter and others, 2017).

Because of the west-to-east gradients in elevation and precipitation, land cover differences, and the use of different hydrologic regions in Painter and others (2017), this report divides Kansas into two hydrologic regions. Region 1 encompasses the eastern part of the State, whereas region 2 consists of the western part of Kansas. The boundary of these regions is near the 26-in. and 28-in. precipitation contour lines, shown in figure 5 (Painter and others, 2017). As mentioned before, irrigation associated with groundwater withdrawals is common in the area west of this boundary, and it is likely that these withdrawals affect surface water (Juracek and Eng, 2017).

\section{Previous Studies in Kansas}

Several low-flow and flow-duration studies have been completed for Kansas. An investigation by Furness (1959) developed a method for estimating flow-duration curves for ungaged locations in Kansas for the period of 1921-56 (Perry and others, 2004b). Jordan (1983) revised the maps developed by Furness by including additional streamgages and data for the period of 1957-76 (Perry and others, 2004b). Two studies completed by Studley $(2000,2001)$ assessed the use of the Furness method to estimate flow-duration curves for ungaged stream locations in Kansas using information from nearby streamgages. The findings of the studies by Studley concluded that the Furness method was a beneficial tool for estimating flow-duration curves at ungaged locations with small drainage areas, less than 100 square miles $\left(\mathrm{mi}^{2}\right)$ (Perry and others, 2004b). Two studies by Perry and others (2004a, b) estimated median flows, flow-duration curves, and peak streamflow for streamgages on the 1999 Kansas Surface Water Register. The Perry and others (2004a) report provided regression equations for mean annual flow; 10-, 25-, 50-, 75-, and 90-percent flow durations; and 2-, 5-, 10-, 25-, 50-, and 100-year (50-, 20-, 10-, 4-, 2-, and 1-percent annual exceedance intervals) peak streamflow flows for drainage areas ranging from 0.17 to $9,100 \mathrm{mi}^{2}$.

\section{Methods for Streamgage Selection}

The data for the initial dataset used for regression equation development in this report were compiled from a total of 351 active and inactive continuous-record streamgages, of which 212 were in Kansas and 139 were from a 50-mi buffer of the neighboring States of Nebraska, Missouri, Oklahoma, and Colorado (fig. 1 and tables 1-3). Streamgages with at least 10 years of record, or 3,650 daily values of discharge, initially were selected for evaluation in this study. Streamgages from neighboring States were used to improve the calculation of selected low-flow frequency statistics for streamgages near the Kansas border. The annual period used in this report for the calculation of annual low-flow frequency statistics was defined as the climatic year (April 1 through March 31). The climatic year is used for low-flow frequency studies because low-flow events in Kansas normally occur during the late summer through winter months. Daily mean streamflow data collected through climatic year 2017 (through March 31, 2017) were used for the regression equation analysis. Daily mean streamflow data collected through water year 2017 (through September 30, 2017) were used for the flow-duration curve analysis in this report. A water year begins on October 1 of the previous calendar year and ends on September 30 of the year of interest. The data for the streamgages in this study were retrieved from the USGS National Water Information System (NWIS) database, which is available at https://doi.org/ 10.5066/F7P55KJN (U.S. Geological Survey, 2018). These data were used to compute selected low-flow frequency and flow-duration statistics.

Peak streamflow data were retrieved from the USGS NWIS database and reviewed to exclude data affected by diversions, regulations, and urbanization. This was done so the computations of selected low-flow and flow-duration statistics would not be biased. The criteria for including a streamgage for the regression equations in this study were location within the study area and a minimum of 10 years of daily streamflow data that were not affected by regulation, diversion, or urbanization. Streamgages with long periods of record sometimes have parts of record that are marked as being affected by regulation, diversion, or urbanization and other parts that are not. If the earlier part of the record period had at least 10 years of daily streamflow data that were not marked as being affected by regulation, diversion, or urbanization, then that part of the record was included in this study. Information about a streamgage being affected by regulation, diversion, or urbanization may be limited or unavailable. It is possible that some data that were affected by one of those activities could have been included in the dataset for this report; however, the overall effect on the computation of the low-flow and flow-duration curve statistics and the development of regression equations is believed to be minimal. Streamgages that lacked basin characteristic data were removed from the regression equation analysis. Streamgages that contained basin characteristic data that were calculated to be zero also were removed from the regression analysis. These streamgages were removed because of the complications that occur when $\log$ transforming zero values. Four streamgages were removed from the regression analysis because the base-flow index (BFI) basin characteristic was calculated to be zero, and three streamgages were removed because the reservoir storage basin characteristic was calculated to be zero.

Streamflow data from streamgages on the Kansas and Arkansas Rivers were not included in the development of the regression equations in this study. The streamflow for the Kansas River streamgages is heavily regulated by upstream reservoirs (fig. 6 and table 4). Factors such as local changes in land and water use, and groundwater withdrawals, are responsible for streamflow alteration along the western reaches 
Table 1. Active and inactive U.S. Geological Survey streamgages used for regression analysis within the study area (map numbers 1-85). U.S. Geological Survey streamgage information is from U.S. Geological Survey (2018).

[no., number; USGS, U.S. Geological Survey; NE, Nebraska; MO, Missouri; C, Creek; NR, near; KS, Kansas; CO, Colorado; SF, South Fork; R, River; ST, State; AB, above; NF, North Fork; BL, below; RE, Reservoir]

\begin{tabular}{|c|c|c|c|c|c|c|}
\hline $\begin{array}{l}\text { Map no. } \\
\text { (fig. 1) }\end{array}$ & $\begin{array}{l}\text { USGS station } \\
\text { number }\end{array}$ & $\begin{array}{l}\text { USGS station } \\
\text { name }\end{array}$ & Latitude & Longitude & No. annual series & $\begin{array}{l}\text { Hydrologic } \\
\text { region }\end{array}$ \\
\hline 1 & 06767500 & PLUM CREEK NEAR SMITHFIELD, NE & 40.64138889 & -99.71055556 & 55 & West \\
\hline 2 & 06768020 & SPRING CREEK NEAR OVERTON, NE & 40.70723067 & -99.5598289 & 21 & West \\
\hline 3 & 06769525 & ELM CREEK NEAR ELM CREEK, NE & 40.7288988 & -99.3989885 & 21 & West \\
\hline 4 & 06810500 & $\begin{array}{l}\text { LITTLE NEMAHA RIVER NEAR SYRACUSE, } \\
\text { NE }\end{array}$ & 40.6325 & -96.179444 & 17 & East \\
\hline 5 & 06811500 & LITTLE NEMAHA RIVER AT AUBURN, NE & 40.39277778 & -95.8127778 & 67 & East \\
\hline 6 & 06813000 & TARKIO RIVER AT FAIRFAX, MO & 40.3391833 & -95.4059056 & 95 & East \\
\hline 7 & 06814000 & TURKEY C NR SENECA, KS & 39.9477773 & -96.1086149 & 68 & East \\
\hline 8 & 06814500 & $\begin{array}{l}\text { NORTH FORK BIG NEMAHA RIVER AT } \\
\text { HUMBOLDT, NE }\end{array}$ & 40.15694485 & -95.9447206 & 64 & East \\
\hline 9 & 06815000 & BIG NEMAHA RIVER AT FALLS CITY, NE & 40.0355577 & -95.5960957 & 73 & East \\
\hline 10 & 06815500 & MUDDY CREEK AT VERDON, NE & 40.14555556 & -95.72027778 & 19 & East \\
\hline 11 & 06817500 & $\begin{array}{l}\text { NODAWAY RIVER NEAR BURLINGTON } \\
\text { JUNCTION, MO }\end{array}$ & 40.44488056 & -95.0888972 & 95 & East \\
\hline 12 & 06817700 & NODAWAY RIVER NEAR GRAHAM, MO & 40.2024722 & -95.0695556 & 34 & East \\
\hline 13 & 06818900 & PLATTE RIVER AT RAVENWOOD, MO & 40.3449905 & -94.6863546 & 12 & East \\
\hline 14 & 06819500 & $\begin{array}{l}\text { ONE HUNDRED AND TWO RIVER AT } \\
\text { MARYVILLE, MO }\end{array}$ & 40.3455 & -94.8321944 & 84 & East \\
\hline 15 & 06820500 & PLATTE RIVER NEAR AGENCY, MO & 39.68802778 & -94.7026111 & 92 & East \\
\hline 16 & 06821080 & $\begin{array}{l}\text { LITTLE PLATTE RIVER NEAR PLATTS- } \\
\text { BURG, MO }\end{array}$ & 39.56777778 & -94.407 & 17 & East \\
\hline 17 & 06821150 & $\begin{array}{l}\text { LITTLE PLATTE RIVER AT SMITHVILLE, } \\
\text { MO }\end{array}$ & 39.38805536 & -94.5791215 & 10 & East \\
\hline 18 & 06821190 & PLATTE RIVER AT SHARPS STATION, MO & 39.4009722 & -94.7268333 & 38 & East \\
\hline 19 & 06823500 & BUFFALO CREEK NEAR HAIGLER, NE & 40.03944444 & -101.8666667 & 76 & West \\
\hline 20 & 06825000 & $\begin{array}{l}\text { SOUTH FORK REPUBLICAN RIVER NEAR } \\
\text { IDALIA, CO }\end{array}$ & 39.6163822 & -102.2426918 & 20 & West \\
\hline 21 & 06825500 & LANDSMAN CREEK NEAR HALE, CO & 39.575549 & -102.2521363 & 25 & West \\
\hline 22 & 06827000 & SF REPUBLICAN R NR CO-KS ST LINE, KS & 39.67193827 & -102.0135164 & 71 & West \\
\hline 23 & 06827500 & $\begin{array}{l}\text { SOUTH FORK REPUBLICAN RIVER NEAR } \\
\text { BENKELMAN, NE }\end{array}$ & 40.01027778 & -101.5419444 & 80 & West \\
\hline
\end{tabular}


Table 1. Active and inactive U.S. Geological Survey streamgages used for regression analysis within the study area (map numbers 1-85). U.S. Geological Survey streamgage information is from U.S. Geological Survey (2018).-Continued

[no., number; USGS, U.S. Geological Survey; NE, Nebraska; MO, Missouri; C, Creek; NR, near; KS, Kansas; CO, Colorado; SF, South Fork; R, River; ST, State; AB, above; NF, North Fork; BL, below; RE, Reservoir]

\begin{tabular}{|c|c|c|c|c|c|c|}
\hline $\begin{array}{l}\text { Map no. } \\
\text { (fig. 1) }\end{array}$ & $\begin{array}{l}\text { USGS station } \\
\text { number }\end{array}$ & $\begin{array}{l}\text { USGS station } \\
\text { name }\end{array}$ & Latitude & Longitude & No. annual series & $\begin{array}{l}\text { Hydrologic } \\
\text { region }\end{array}$ \\
\hline 24 & 06828000 & REPUBLICAN RIVER AT MAX, NE & 40.1016622 & -101.3998866 & 15 & West \\
\hline 25 & 06835000 & $\begin{array}{l}\text { STINKING WATER CREEK NEAR PALI- } \\
\text { SADE, NE }\end{array}$ & 40.373056 & -101.116944 & 44 & West \\
\hline 26 & 06836000 & $\begin{array}{l}\text { BLACKWOOD CREEK NEAR CULBERTSON, } \\
\text { NE }\end{array}$ & 40.23611111 & -100.8111111 & 39 & West \\
\hline 27 & 06836500 & DRIFTWOOD CREEK NEAR MCCOOK, NE & 40.14583655 & -100.6732083 & 71 & West \\
\hline 28 & 06837300 & $\begin{array}{l}\text { RED WILLOW CREEK ABOVE HUGH BUT- } \\
\text { LER LAKE, NE }\end{array}$ & 40.4013948 & -100.7837665 & 33 & West \\
\hline 29 & 06838000 & $\begin{array}{l}\text { RED WILLOW CREEK NEAR RED WILLOW, } \\
\text { NE }\end{array}$ & 40.2347222 & -100.5008333 & 21 & West \\
\hline 30 & 06840000 & FOX CREEK AT CURTIS, NE & 40.634722 & -100.490278 & 40 & West \\
\hline 31 & 06841000 & $\begin{array}{l}\text { MEDICINE CREEK ABOVE HARRY STRUNK } \\
\text { LAKE, NE }\end{array}$ & 40.500556 & -100.322778 & 44 & West \\
\hline 32 & 06843000 & MEDICINE CREEK AT CAMBRIDGE, NE & 40.298333 & -100.176944 & 12 & West \\
\hline 33 & 06844000 & MUDDY CREEK AT ARAPAHOE, NE & 40.305833 & -99.911667 & 42 & West \\
\hline 34 & 06844210 & TURKEY CREEK AT EDISON, NE & 40.2708411 & -99.7337279 & 15 & West \\
\hline 35 & 06844900 & SF SAPPA C NR ACHILLES, KS & 39.6769485 & -100.7220931 & 49 & West \\
\hline 36 & 06845000 & SAPPA C NR OBERLIN, KS & 39.8130606 & -100.5343122 & 77 & West \\
\hline 37 & 06845110 & SAPPA C NR LYLE, KS & 40.0016726 & -99.9929055 & 21 & West \\
\hline 38 & 06845200 & SAPPA CREEK NEAR BEAVER CITY, NE & 40.04584 & -99.8904005 & 64 & West \\
\hline 39 & 06846000 & BEAVER C AT LUDELL, KS & 39.8480579 & -100.9615446 & 81 & West \\
\hline 40 & 06846500 & BEAVER C AT CEDAR BLUFFS, KS & 39.98500557 & -100.560148 & 70 & West \\
\hline 41 & 06847000 & BEAVER CREEK NEAR BEAVER CITY, NE & 40.1200068 & -99.8934566 & 79 & West \\
\hline 42 & 06847500 & SAPPA CREEK NEAR STAMFORD, NE & 40.1183333 & -99.5169444 & 71 & West \\
\hline 43 & 06847900 & $\begin{array}{l}\text { PRAIRIE DOG C AB KEITH SEBELIUS } \\
\text { LAKE, KS }\end{array}$ & 39.769727 & -100.1006875 & 54 & West \\
\hline 44 & 06848000 & PRAIRIE DOG C AT NORTON, KS & 39.8100055 & -99.9220683 & 19 & West \\
\hline 45 & 06848500 & PRAIRIE DOG C NR WOODRUFF, KS & 39.9787043 & -99.4786584 & 34 & West \\
\hline 46 & 06850500 & $\begin{array}{l}\text { REPUBLICAN RIVER NEAR BLOOMING- } \\
\text { TON, NE }\end{array}$ & 40.066389 & -99.036389 & 20 & West \\
\hline 47 & 06851000 & CENTER CREEK AT FRANKLIN, NE & 40.103333 & -98.980278 & 45 & West \\
\hline
\end{tabular}


Table 1. Active and inactive U.S. Geological Survey streamgages used for regression analysis within the study area (map numbers 1-85). U.S. Geological Survey streamgage information is from U.S. Geological Survey (2018)._-Continued

[no., number; USGS, U.S. Geological Survey; NE, Nebraska; MO, Missouri; C, Creek; NR, near; KS, Kansas; CO, Colorado; SF, South Fork; R, River; ST, State; AB, above; NF, North Fork; BL, below; RE, Reservoir]

\begin{tabular}{|c|c|c|c|c|c|c|}
\hline $\begin{array}{l}\text { Map no. } \\
\text { (fig. 1) }\end{array}$ & $\begin{array}{c}\text { USGS station } \\
\text { number }\end{array}$ & $\begin{array}{l}\text { USGS station } \\
\text { name }\end{array}$ & Latitude & Longitude & No. annual series & $\begin{array}{l}\text { Hydrologic } \\
\text { region }\end{array}$ \\
\hline 48 & 06851500 & THOMPSON CREEK AT RIVERTON, NE & 40.0891807 & -98.7609064 & 46 & West \\
\hline 49 & 06853500 & REPUBLICAN R NR HARDY, NE & 39.992513 & -97.932543 & 46 & East \\
\hline 50 & 06853800 & WHITE ROCK C NR BURR OAK, KS & 39.89918 & -98.2503276 & 59 & East \\
\hline 51 & 06854500 & REPUBLICAN R AT SCANDIA, KS & 39.79873 & -97.7931266 & 31 & East \\
\hline 52 & 06855800 & BUFFALO C NR JAMESTOWN, KS & 39.6145692 & -97.8568182 & 30 & East \\
\hline 53 & 06855900 & WOLF C NR CONCORDIA, KS & 39.5437887 & -97.723024 & 19 & East \\
\hline 54 & 06856600 & REPUBLICAN R AT CLAY CENTER, KS & 39.3555515 & -97.1275203 & 33 & East \\
\hline 55 & 06857000 & REPUBLICAN R AT MILFORD, KS & 39.1647189 & -96.9158446 & 57 & East \\
\hline 56 & 06858500 & NF SMOKY HILL R NR MCALLASTER, KS & 39.01787498 & -101.3479109 & 37 & West \\
\hline 57 & 06859500 & $\begin{array}{l}\text { LADDER C BL CHALK C NR SCOTT CITY, } \\
\text { KS }\end{array}$ & 38.7889022 & -100.8698688 & 28 & West \\
\hline 58 & 06860000 & SMOKY HILL R AT ELKADER, KS & 38.7947354 & -100.8584796 & 77 & West \\
\hline 59 & 06861000 & SMOKY HILL R NR ARNOLD, KS & 38.8077898 & -100.0226243 & 67 & West \\
\hline 60 & 06862500 & SMOKY HILL R NR ELLIS, KS & 38.76862365 & -99.560034 & 71 & West \\
\hline 61 & 06863300 & BIG C NR OGALLAH, KS & 38.9106938 & -99.7433365 & 12 & West \\
\hline 62 & 06863500 & BIG C NR HAYS, KS & 38.852234 & -99.3181589 & 71 & West \\
\hline 63 & 06863900 & NF BIG C NR VICTORIA, KS & 38.8866783 & -99.2062101 & 25 & West \\
\hline 64 & 06864000 & SMOKY HILL R NR RUSSELL, KS & 38.7766781 & -98.8548066 & 10 & West \\
\hline 65 & 06864500 & SMOKY HILL R AT ELLSWORTH, KS & 38.72667589 & -98.2336684 & 54 & East \\
\hline 66 & 06866000 & SMOKY HILL R AT LINDSBORG, KS & 38.56380659 & -97.6664947 & 41 & East \\
\hline 67 & 06867000 & SALINE R NR RUSSELL, KS & 38.96584479 & -98.8545276 & 71 & West \\
\hline 68 & 06867500 & PARADISE C NR PARADISE, KS & 39.05919167 & -98.8422917 & 71 & West \\
\hline 69 & 06868000 & SALINE R NR WILSON, KS & 38.9333444 & -98.53368 & 33 & East \\
\hline 70 & 06868400 & WOLF C NR LUCAS, KS & 39.0576803 & -98.5597071 & 11 & East \\
\hline 71 & 06869500 & SALINE R AT TESCOTT, KS & 39.0038923 & -97.8739316 & 43 & East \\
\hline 72 & 06869950 & MULBERRY C NR SALINA, KS & 38.8444476 & -97.6683699 & 15 & East \\
\hline 73 & 06871000 & NF SOLOMON R AT GLADE, KS & 39.6730652 & -99.3095409 & 64 & West \\
\hline 74 & 06871500 & BOW C NR STOCKTON, KS & 39.5594542 & -99.2859298 & 66 & West \\
\hline 75 & 06871800 & NF SOLOMON R AT KIRWIN, KS & 39.6600106 & -99.115644 & 34 & West \\
\hline 76 & 06871900 & DEER C NR PHILLIPSBURG, KS & 39.7810731 & -99.4221617 & 14 & West \\
\hline
\end{tabular}


Table 1. Active and inactive U.S. Geological Survey streamgages used for regression analysis within the study area (map numbers 1-85). U.S. Geological Survey streamgage information is from U.S. Geological Survey (2018).-Continued

[no., number; USGS, U.S. Geological Survey; NE, Nebraska; MO, Missouri; C, Creek; NR, near; KS, Kansas; CO, Colorado; SF, South Fork; R, River; ST, State; AB, above; NF, North Fork; BL, below; RE, Reservoir]

\begin{tabular}{|c|c|c|c|c|c|c|}
\hline $\begin{array}{c}\text { Map no. } \\
\text { (fig. 1) }\end{array}$ & $\begin{array}{c}\text { USGS station } \\
\text { number }\end{array}$ & $\begin{array}{l}\text { USGS station } \\
\text { name }\end{array}$ & Latitude & Longitude & No. annual series & $\begin{array}{l}\text { Hydrologic } \\
\text { region }\end{array}$ \\
\hline 77 & 06873000 & SF SOLOMON R AB WEBSTER RE, KS & 39.37667456 & -99.5801096 & 72 & West \\
\hline 78 & 06873500 & SF SOLOMON R AT ALTON, KS & 39.4543049 & -98.9483529 & 37 & West \\
\hline 79 & 06873700 & KILL C NR BLOOMINGTON, KS & 39.379177 & -98.8595249 & 18 & West \\
\hline 80 & 06876000 & SOLOMON R AT BELOIT, KS & 39.45450957 & -98.1098985 & 25 & East \\
\hline 81 & 06876700 & SALT C NR ADA, KS & 39.13916876 & -97.8369849 & 57 & East \\
\hline 82 & 06876900 & SOLOMON R AT NILES, KS & 38.9691663 & -97.4772513 & 56 & East \\
\hline 83 & 06877000 & SMOKY HILL R AT SOLOMON, KS & 38.9051028 & -97.3683676 & 15 & East \\
\hline 84 & 06877600 & SMOKY HILL R AT ENTERPRISE, KS & 38.90638906 & -97.1177952 & 11 & East \\
\hline 85 & 06878000 & CHAPMAN C NR CHAPMAN, KS & 39.031109 & -97.0402922 & 63 & East \\
\hline
\end{tabular}


Table 2. Active and inactive U.S. Geological Survey streamgages used for regression analysis within the study area (map numbers 86-170). U.S. Geological Survey streamgage information is from U.S. Geological Survey (2018).

[no., number; USGS, U.S. Geological Survey; C, Creek; NR, near; KS, Kansas; NE, Nebraska; R, River; L, Little; ST., Saint; ST, Street; BLVD, Boulevard; EXT, Exit; KC, Kansas City; MO, Missouri; CO, Colorado]

\begin{tabular}{|c|c|c|c|c|c|c|}
\hline $\begin{array}{l}\text { Map no. } \\
\text { (fig. 1) }\end{array}$ & $\begin{array}{l}\text { USGS station } \\
\text { number }\end{array}$ & $\begin{array}{l}\text { USGS station } \\
\text { name }\end{array}$ & Latitude & Longitude & No. annual series & $\begin{array}{l}\text { Hydrologic } \\
\text { region }\end{array}$ \\
\hline 86 & 06878500 & LYON C NR WOODBINE, KS & 38.8847238 & -96.9100115 & 20 & East \\
\hline 87 & 06881000 & BIG BLUE RIVER NEAR CRETE, NE & 40.59666667 & -96.9605556 & 63 & East \\
\hline 88 & 06881200 & TURKEY CREEK NEAR WILBER, NE & 40.480004 & -97.0122523 & 34 & East \\
\hline 89 & 06881380 & TURKEY CREEK NEAR DEWITT, NE & 40.3672222 & -96.8894444 & 14 & East \\
\hline 90 & 06881500 & BIG BLUE R AT BEATRICE, NE & 40.256667 & -96.746944 & 83 & East \\
\hline 91 & 06882000 & BIG BLUE RIVER AT BARNESTON, NE & 40.0447222 & -96.5872222 & 84 & East \\
\hline 92 & 06882500 & BIG BLUE R AT HULL, KS & 39.9166688 & -96.6336319 & 20 & East \\
\hline 93 & 06882510 & BIG BLUE R AT MARYSVILLE, KS & 39.8419463 & -96.6622425 & 32 & East \\
\hline 94 & 06883000 & $\begin{array}{l}\text { LITTLE BLUE RIVER NEAR DEWEESE, } \\
\text { NE }\end{array}$ & 40.3325 & -98.0669444 & 64 & East \\
\hline 95 & 06883570 & $\begin{array}{l}\text { LITTLE BLUE RIVER NEAR ALEXAN- } \\
\text { DRIA (GILEAD), NE }\end{array}$ & 40.206944 & -97.388611 & 32 & East \\
\hline 96 & 06884000 & $\begin{array}{l}\text { LITTLE BLUE RIVER NEAR FAIRBURY, } \\
\text { NE }\end{array}$ & 40.1464722 & -97.2007222 & 107 & East \\
\hline 97 & 06884025 & $\begin{array}{l}\text { LITTLE BLUE RIVER AT HOLLENBERG, } \\
\text { KS }\end{array}$ & 39.98027778 & -97.0047222 & 43 & East \\
\hline 98 & 06884200 & MILL C AT WASHINGTON, KS & 39.81361476 & -97.0375267 & 57 & East \\
\hline 99 & 06884400 & L BLUE R NR BARNES, KS & 39.72583518 & -96.8047432 & 58 & East \\
\hline 100 & 06884500 & L BLUE R AT WATERVILLE, KS & 39.7004873 & -96.7545251 & 35 & East \\
\hline 101 & 06885500 & $\begin{array}{l}\text { BLACK VERMILLION R NR FRANKFORT, } \\
\text { KS }\end{array}$ & 39.68194349 & -96.4427888 & 63 & East \\
\hline 102 & 06886000 & BIG BLUE R AT RANDOLPH, KS & 39.4765097 & -96.6887196 & 41 & East \\
\hline 103 & 06888000 & VERMILLION C NR WAMEGO, KS & 39.34777386 & -96.2174979 & 80 & East \\
\hline 104 & 06888500 & MILL C NR PAXICO, KS & 39.06472106 & -96.1691605 & 63 & East \\
\hline 105 & 06889120 & SOLDIER C NR BANCROFT, KS & 39.59476 & -95.97391908 & 24 & East \\
\hline 106 & 06889140 & SOLDIER C NR SOLDIER, KS & 39.5768743 & -95.9756814 & 34 & East \\
\hline 107 & 06889160 & SOLDIER C NR CIRCLEVILLE, KS & 39.4631449 & -95.9505944 & 37 & East \\
\hline 108 & 06889180 & SOLDIER C NR ST. CLERE, KS & 39.3758289 & -95.9183232 & 17 & East \\
\hline 109 & 06889200 & SOLDIER C NR DELIA, KS & 39.23833024 & -95.8885992 & 58 & East \\
\hline 110 & 06889500 & SOLDIER C NR TOPEKA, KS & 39.0994439 & -95.7249828 & 87 & East \\
\hline 111 & 06890100 & DELAWARE R NR MUSCOTAH, KS & 39.5213873 & -95.532756 & 47 & East \\
\hline
\end{tabular}


Table 2. Active and inactive U.S. Geological Survey streamgages used for regression analysis within the study area (map numbers 86-170). U.S. Geological Survey streamgage information is from U.S. Geological Survey (2018).-Continued

[no., number; USGS, U.S. Geological Survey; C, Creek; NR, near; KS, Kansas; NE, Nebraska; R, River; L, Little; ST., Saint; ST, Street; BLVD, Boulevard; EXT, Exit; KC, Kansas City; MO, Missouri; CO, Colorado]

\begin{tabular}{|c|c|c|c|c|c|c|}
\hline $\begin{array}{c}\text { Map no. } \\
\text { (fig. 1) }\end{array}$ & $\begin{array}{c}\text { USGS station } \\
\text { number }\end{array}$ & $\begin{array}{l}\text { USGS station } \\
\text { name }\end{array}$ & Latitude & Longitude & No. annual series & $\begin{array}{l}\text { Hydrologic } \\
\text { region }\end{array}$ \\
\hline 112 & 06890500 & DELAWARE R AT VALLEY FALLS, KS & 39.3508317 & -95.4546974 & 44 & East \\
\hline 113 & 06891260 & WAKARUSA R NR RICHLAND, KS & 38.89194444 & -95.5944444 & 14 & East \\
\hline 114 & 06891500 & WAKARUSA R NR LAWRENCE, KS & 38.9113923 & -95.261084 & 51 & East \\
\hline 115 & 06891810 & STRANGER C NR POTTER, KS & 39.4477753 & -95.162191 & 11 & East \\
\hline 116 & 06892000 & STRANGER C NR TONGANOXIE, KS & 39.1163912 & -95.0108006 & 87 & East \\
\hline 117 & 06892360 & KILL C AT 95TH ST NR DESOTO, KS & 38.95666667 & -94.9736111 & 14 & East \\
\hline 118 & 06893080 & BLUE R NR STANLEY, KS & 38.8125082 & -94.6757906 & 42 & East \\
\hline 119 & 06893150 & $\begin{array}{l}\text { BLUE RIVER AT BLUE RIDGE BLVD EXT } \\
\text { IN KC, MO }\end{array}$ & 38.88941667 & -94.5806667 & 14 & East \\
\hline 120 & 06893300 & INDIAN C AT OVERLAND PARK, KS & 38.9405621 & -94.6713469 & 16 & East \\
\hline 121 & 06893500 & BLUE RIVER AT KANSAS CITY, MO & 38.957 & -94.5588889 & 77 & East \\
\hline 122 & 06893578 & $\begin{array}{l}\text { BLUE RIVER AT STADIUM DRIVE IN } \\
\text { KANSAS CITY, MO }\end{array}$ & 39.0583384 & -94.5118983 & 14 & East \\
\hline 123 & 06893793 & $\begin{array}{l}\text { LITTLE BLUE RIVER BELOW LONGVIEW } \\
\text { DAM AT KC, MO }\end{array}$ & 38.925917 & -94.468583 & 17 & East \\
\hline 124 & 06893890 & $\begin{array}{l}\text { EAST FORK LITTLE BLUE RIVER NEAR } \\
\text { BLUE SPRINGS, MO }\end{array}$ & 39.02556175 & -94.3438385 & 42 & East \\
\hline 125 & 06894000 & $\begin{array}{l}\text { LITTLE BLUE RIVER NEAR LAKE CITY, } \\
\text { MO }\end{array}$ & 39.1005609 & -94.3005038 & 69 & East \\
\hline 126 & 06894500 & $\begin{array}{l}\text { EAST FORK FISHING RIVER AT EXCEL- } \\
\text { SIOR SPRINGS, MO }\end{array}$ & 39.338781 & -94.213183 & 19 & East \\
\hline 127 & 06895000 & CROOKED RIVER NEAR RICHMOND, MO & 39.33266389 & -93.98 & 69 & East \\
\hline 128 & 06896500 & $\begin{array}{l}\text { THOMPSON BRANCH NEAR ALBANY, } \\
\text { MO }\end{array}$ & 40.213964 & -94.332111 & 16 & East \\
\hline 129 & 06897000 & $\begin{array}{l}\text { EAST FORK BIG CREEK NEAR BETHA- } \\
\text { NY, MO }\end{array}$ & 40.2972222 & -94.0262222 & 83 & East \\
\hline 130 & 06897500 & GRAND RIVER NEAR GALLATIN, MO & 39.9269517 & -93.9427225 & 95 & East \\
\hline 131 & 06898100 & $\begin{array}{l}\text { THOMPSON RIVER AT MOUNT MORIAH, } \\
\text { MO }\end{array}$ & 40.336333 & -93.7685 & 16 & East \\
\hline 132 & 06899700 & SHOAL CREEK NEAR BRAYMER, MO & 39.699525 & -93.7958722 & 59 & East \\
\hline 133 & 06907500 & $\begin{array}{l}\text { SOUTH FORK BLACKWATER RIVER } \\
\text { NEAR ELM, MO }\end{array}$ & 38.818956 & -94.035864 & 24 & East \\
\hline
\end{tabular}


Table 2. Active and inactive U.S. Geological Survey streamgages used for regression analysis within the study area (map numbers 86-170). U.S. Geological Survey streamgage information is from U.S. Geological Survey (2018)._-Continued

[no., number; USGS, U.S. Geological Survey; C, Creek; NR, near; KS, Kansas; NE, Nebraska; R, River; L, Little; ST., Saint; ST, Street; BLVD, Boulevard; EXT, Exit; KC, Kansas City; MO, Missouri; CO, Colorado]

\begin{tabular}{|c|c|c|c|c|c|c|}
\hline $\begin{array}{l}\text { Map no. } \\
\text { (fig. 1) }\end{array}$ & $\begin{array}{l}\text { USGS station } \\
\text { number }\end{array}$ & $\begin{array}{l}\text { USGS station } \\
\text { name }\end{array}$ & Latitude & Longitude & No. annual series & $\begin{array}{l}\text { Hydrologic } \\
\text { region }\end{array}$ \\
\hline 134 & 06910800 & $\begin{array}{l}\text { MARAIS DES CYGNES R NR READING, } \\
\text { KS }\end{array}$ & 38.56695524 & -95.961657 & 47 & East \\
\hline 135 & 06911000 & $\begin{array}{l}\text { MARAIS DES CYGNES R AT MELVERN, } \\
\text { KS }\end{array}$ & 38.5161111 & -95.6961111 & 32 & East \\
\hline 136 & 06911490 & SALT C AT LYNDON, KS & 38.60139907 & -95.6847068 & 17 & East \\
\hline 137 & 06911500 & SALT C NR LYNDON, KS & 38.6088989 & -95.6383168 & 59 & East \\
\hline 138 & 06911900 & DRAGOON C NR BURLINGAME, KS & 38.7091737 & -95.8383205 & 57 & East \\
\hline 139 & 06912500 & $\begin{array}{l}\text { HUNDRED AND TEN MILE C NR QUEN- } \\
\text { EMO, KS }\end{array}$ & 38.6450089 & -95.5597037 & 23 & East \\
\hline 140 & 06913000 & $\begin{array}{l}\text { MARAIS DES CYGNES R NR POMONA, } \\
\text { KS }\end{array}$ & 38.5841777 & -95.4535902 & 14 & East \\
\hline 141 & 06913500 & MARAIS DES CYGNES R NR OTTAWA, KS & 38.61806669 & -95.2683071 & 60 & East \\
\hline 142 & 06914000 & POTTAWATOMIE C NR GARNETT, KS & 38.333631 & -95.2488634 & 77 & East \\
\hline 143 & 06914100 & POTTAWATOMIE C NR SCIPIO, KS & 38.348889 & -95.203333 & 15 & East \\
\hline 144 & 06914950 & BIG BULL C NR EDGERTON, KS & 38.75334168 & -94.977188 & 23 & East \\
\hline 145 & 06914990 & L BULL C NR SPRING HILL, KS & 38.7675084 & -94.8794073 & 23 & East \\
\hline 146 & 06915000 & BIG BULL C NR HILLSDALE, KS & 38.6563999 & -94.8963521 & 21 & East \\
\hline 147 & 06916000 & $\begin{array}{l}\text { MARAIS DES CYGNES R AT TRADING } \\
\text { POST, KS }\end{array}$ & 38.2486687 & -94.686432 & 29 & East \\
\hline 148 & 06916500 & BIG SUGAR C AT FARLINVILLE, KS & 38.2353027 & -94.8538516 & 41 & East \\
\hline 149 & 06917000 & L OSAGE R AT FULTON, KS & 38.00892428 & -94.7041264 & 68 & East \\
\hline 150 & 06917060 & LITTLE OSAGE RIVER AT HORTON, MO & 37.99480556 & -94.3693056 & 28 & East \\
\hline 151 & 06917240 & MARMATON R NR UNIONTOWN, KS & 37.83559565 & -94.981361 & 16 & East \\
\hline 152 & 06917380 & MARMATON R NR MARMATON, KS & 37.81754116 & -94.7919095 & 36 & East \\
\hline 153 & 06917500 & MARMATON R NR FORT SCOTT, KS & 37.848929 & -94.7027392 & 95 & East \\
\hline 154 & 06917560 & $\begin{array}{l}\text { MARMATON RIVER NEAR RICHARDS, } \\
\text { MO }\end{array}$ & 37.8647222 & -94.5822222 & 11 & East \\
\hline 155 & 06918060 & MARMATON RIVER NEAR NEVADA, MO & 37.86202778 & -94.39925 & 13 & East \\
\hline 156 & 06918070 & OSAGE RIVER ABOVE SCHELL CITY, MO & 38.0558611 & -94.1454167 & 29 & East \\
\hline 157 & 06918460 & $\begin{array}{l}\text { TURNBACK CREEK ABOVE GREEN- } \\
\text { FIELD, MO }\end{array}$ & 37.4023611 & -93.8020278 & 51 & East \\
\hline
\end{tabular}


Table 2. Active and inactive U.S. Geological Survey streamgages used for regression analysis within the study area (map numbers 86-170). U.S. Geological Survey streamgage information is from U.S. Geological Survey (2018).-Continued

[no., number; USGS, U.S. Geological Survey; C, Creek; NR, near; KS, Kansas; NE, Nebraska; R, River; L, Little; ST., Saint; ST, Street; BLVD, Boulevard; EXT, Exit; KC, Kansas City; MO, Missouri; CO, Colorado]

\begin{tabular}{|c|c|c|c|c|c|c|}
\hline $\begin{array}{l}\text { Map no. } \\
\text { (fig. 1) }\end{array}$ & $\begin{array}{l}\text { USGS station } \\
\text { number }\end{array}$ & $\begin{array}{l}\text { USGS station } \\
\text { name }\end{array}$ & Latitude & Longitude & No. annual series & $\begin{array}{l}\text { Hydrologic } \\
\text { region }\end{array}$ \\
\hline 158 & 06919000 & SAC RIVER NEAR STOCKTON, MO & 37.70105278 & -93.7567806 & 46 & East \\
\hline 159 & 06919500 & $\begin{array}{l}\text { CEDAR CREEK NEAR PLEASANT VIEW, } \\
\text { MO }\end{array}$ & 37.83420277 & -93.8754918 & 93 & East \\
\hline 160 & 06921590 & SOUTH GRAND RIVER AT ARCHIE, MO & 38.49158056 & -94.3442444 & 47 & East \\
\hline 161 & 06921600 & SOUTH GRAND RIVER AT URICH, MO & 38.45223976 & -94.0043859 & 56 & East \\
\hline 162 & 06921720 & BIG CREEK NEAR BLAIRSTOWN, MO & 38.55497778 & -93.9653528 & 56 & East \\
\hline 163 & 06921760 & $\begin{array}{l}\text { SOUTH GRAND RIVER NEAR CLINTON, } \\
\text { MO }\end{array}$ & 38.370139 & -93.858111 & 21 & East \\
\hline 164 & 06922000 & $\begin{array}{l}\text { SOUTH GRAND RIVER NEAR BROWN- } \\
\text { INGTON, MO }\end{array}$ & 38.263611 & -93.714444 & 49 & East \\
\hline 165 & 07134100 & BIG SANDY CREEK NEAR LAMAR, CO & 38.11417565 & -102.4838023 & 49 & West \\
\hline 166 & 07139800 & MULBERRY C NR DODGE CITY, KS & 37.5977599 & -100.0153544 & 22 & West \\
\hline 167 & 07140700 & GUZZLERS GULCH NR NESS CITY, KS & 38.2922532 & -99.951842 & 18 & West \\
\hline 168 & 07140850 & PAWNEE R NR BURDETT, KS & 38.2066829 & -99.6434539 & 35 & West \\
\hline 169 & 07141175 & BUCKNER C NR BURDETT, KS & 38.1625166 & -99.6428988 & 17 & West \\
\hline 170 & 07141200 & PAWNEE R AT ROZEL, KS & 38.2075145 & -99.4062247 & 92 & West \\
\hline
\end{tabular}


Table 3. Active and inactive U.S. Geological Survey streamgages used for regression analysis within the study area (map numbers 171-254). U.S. Geological Survey streamgage information is from U.S. Geological Survey (2018).

[no., number; USGS, U.S. Geological Survey; C, Creek; NR, near; KS, Kansas; L, Little; R, River; HWY, Highway; NF, North Fork; AB, above; RE, Reservoir; SF, South Fork; OK, Oklahoma; CLEV, Cleveland; BLW, below; M, Middle; MO, Missouri]

\begin{tabular}{|c|c|c|c|c|c|c|}
\hline $\begin{array}{l}\text { Map no. } \\
\text { (fig. 1) }\end{array}$ & $\begin{array}{c}\text { USGS station } \\
\text { number }\end{array}$ & $\begin{array}{l}\text { USGS station } \\
\text { name }\end{array}$ & Latitude & Longitude & No. annual series & $\begin{array}{l}\text { Hydrologic } \\
\text { region }\end{array}$ \\
\hline 171 & 07141770 & WALNUT C NR ALEXANDER, KS & 38.4647369 & -99.6226162 & 18 & West \\
\hline 172 & 07141780 & WALNUT C AT NEKOMA, KS & 38.47723614 & -99.4381656 & 47 & West \\
\hline 173 & 07141900 & WALNUT C AT ALBERT, KS & 38.46167948 & -99.014817 & 58 & West \\
\hline 174 & 07142300 & RATTLESNAKE C NR MACKSVILLE, KS & 37.8716826 & -98.8762067 & 57 & West \\
\hline 175 & 07142575 & RATTLESNAKE C NR ZENITH, KS & 38.0936248 & -98.5461894 & 43 & East \\
\hline 176 & 07143300 & COW C NR LYONS, KS & 38.3083438 & -98.1920045 & 79 & East \\
\hline 177 & 07143600 & L ARKANSAS R NR LITTLE RIVER, KS & 38.41329 & -98.0173439 & 11 & East \\
\hline 178 & 07143665 & L ARKANSAS R AT ALTA MILLS, KS & 38.1122326 & -97.591987 & 38 & East \\
\hline 179 & 07143672 & $\begin{array}{l}\text { L ARKANSAS R AT HWY } 50 \text { NR HALSTEAD, } \\
\text { KS }\end{array}$ & 38.0286223 & -97.540597 & 21 & East \\
\hline 180 & 07144100 & L ARKANSAS R NR SEDGWICK, KS & 37.88306739 & -97.4244864 & 23 & East \\
\hline 181 & 07144200 & L ARKANSAS R AT VALLEY CENTER, KS & 37.83223425 & -97.3889317 & 94 & East \\
\hline 182 & 07144780 & NF NINNESCAH R AB CHENEY RE, KS & 37.8625129 & -98.0139449 & 51 & East \\
\hline 183 & 07144800 & NF NINNESCAH R NR CHENEY, KS & 37.6675828 & -97.7559865 & 13 & East \\
\hline 184 & 07145200 & SF NINNESCAH R NR MURDOCK, KS & 37.561683 & -97.8531094 & 66 & East \\
\hline 185 & 07145500 & NINNESCAH R NR PECK, KS & 37.4569636 & -97.4239353 & 25 & East \\
\hline 186 & 07145700 & SLATE C AT WELLINGTON, KS & 37.24946707 & -97.4036569 & 48 & East \\
\hline 187 & 07146570 & COLE C NR DEGRAFF, KS & 37.9478819 & -96.7819805 & 19 & East \\
\hline 188 & 07147070 & WHITEWATER R AT TOWANDA, KS & 37.79612785 & -97.0144806 & 51 & East \\
\hline 189 & 07147800 & WALNUT R AT WINFIELD, KS & 37.22391557 & -96.9961475 & 59 & East \\
\hline 190 & 07148350 & $\begin{array}{l}\text { SALT FORK ARKANSAS RIVER NR WIN- } \\
\text { CHESTER, OK }\end{array}$ & 36.9616959 & -98.7823116 & 33 & East \\
\hline 191 & 07148400 & $\begin{array}{l}\text { SALT FORK ARKANSAS RIVER NR ALVA, } \\
\text { OK }\end{array}$ & 36.81503056 & -98.6481395 & 79 & East \\
\hline 192 & 07149000 & MEDICINE LODGE R NR KIOWA, KS & 37.03891547 & -98.4709092 & 79 & East \\
\hline 193 & 07151500 & CHIKASKIA R NR CORBIN, KS & 37.12891225 & -97.6019945 & 66 & East \\
\hline 194 & 07152000 & CHIKASKIA RIVER NEAR BLACKWELL, OK & 36.8114211 & -97.277265 & 41 & East \\
\hline 195 & 07153000 & BLACK BEAR CREEK AT PAWNEE, OK & 36.34366537 & -96.7994788 & 22 & East \\
\hline 196 & 07153100 & $\begin{array}{l}\text { RANCH CREEK AT CLEV DAM NR CLEVE- } \\
\text { LAND, OK }\end{array}$ & 36.2833984 & -96.5766931 & 23 & East \\
\hline 197 & 07155590 & CIMARRON R NR ELKHART, KS & 37.12196746 & -101.8979456 & 39 & West \\
\hline
\end{tabular}


Table 3. Active and inactive U.S. Geological Survey streamgages used for regression analysis within the study area (map numbers 171-254). U.S. Geological Survey streamgage information is from U.S. Geological Survey (2018).-Continued

[no., number; USGS, U.S. Geological Survey; C, Creek; NR, near; KS, Kansas; L, Little; R, River; HWY, Highway; NF, North Fork; AB, above; RE, Reservoir; SF, South Fork; OK, Oklahoma; CLEV, Cleveland; BLW, below; M, Middle; MO, Missouri]

\begin{tabular}{|c|c|c|c|c|c|c|}
\hline $\begin{array}{c}\text { Map no. } \\
\text { (fig. 1) }\end{array}$ & $\begin{array}{c}\text { USGS station } \\
\text { number }\end{array}$ & $\begin{array}{c}\text { USGS station } \\
\text { name }\end{array}$ & Latitude & Longitude & No. annual series & $\begin{array}{l}\text { Hydrologic } \\
\text { region }\end{array}$ \\
\hline 198 & 07156010 & NF CIMARRON R AT RICHFIELD, KS & 37.25835665 & -101.7754438 & 14 & West \\
\hline 199 & 07157000 & CIMARRON RIVER NEAR MOCANE, OK & 36.9758611 & -100.3143115 & 22 & West \\
\hline 200 & 07157500 & CROOKED C NR ENGLEWOOD, KS & 37.032528 & -100.2112531 & 74 & West \\
\hline 201 & 07157950 & CIMARRON RIVER NEAR BUFFALO, OK & 36.85197484 & -99.315387 & 57 & West \\
\hline 202 & 07157960 & BUFFALO CREEK NEAR LOVEDALE, OK & 36.7705872 & -99.3670537 & 26 & West \\
\hline 203 & 07158000 & CIMARRON RIVER NEAR WAYNOKA, OK & 36.51725644 & -98.8795366 & 40 & East \\
\hline 204 & 07159000 & TURKEY CREEK NEAR DRUMMOND, OK & 36.31809 & -98.001172 & 22 & East \\
\hline 205 & 07165700 & VERDIGRIS R NR MADISON, KS & 38.1349719 & -96.1033057 & 20 & East \\
\hline 206 & 07165750 & VERDIGRIS R NR VIRGIL, KS & 37.98252814 & -96.0238817 & 27 & East \\
\hline 207 & 07166500 & VERDIGRIS R NR ALTOONA, KS & 37.5297764 & -95.674703 & 20 & East \\
\hline 208 & 07167000 & FALL R NR EUREKA, KS & 37.7850551 & -96.2348096 & 29 & East \\
\hline 209 & 07167500 & OTTER C AT CLIMAX, KS & 37.70808736 & -96.2236107 & 70 & East \\
\hline 210 & 07168500 & FALL R NR FALL RIVER, KS & 37.6445207 & -96.0570703 & 10 & East \\
\hline 211 & 07169500 & FALL R AT FREDONIA, KS & 37.50838374 & -95.8335924 & 50 & East \\
\hline 212 & 07169800 & ELK R AT ELK FALLS, KS & 37.37559729 & -96.185548 & 44 & East \\
\hline 213 & 07170000 & ELK R NR ELK CITY, KS & 37.2664504 & -95.918041 & 30 & East \\
\hline 214 & 07170500 & VERDIGRIS R AT INDEPENDENCE, KS & 37.22368014 & -95.6777573 & 52 & East \\
\hline 215 & 07170700 & BIG HILL C NR CHERRYVALE, KS & 37.2667325 & -95.469144 & 59 & East \\
\hline 216 & 07171000 & VERDIGRIS RIVER NEAR LENAPAH, OK & 36.85119615 & -95.5860882 & 10 & East \\
\hline 217 & 07172000 & CANEY R NR ELGIN, KS & 37.00393886 & -96.3166638 & 77 & East \\
\hline 218 & 07173000 & CANEY RIVER NEAR HULAH, OK & 36.9184044 & -96.0710968 & 12 & East \\
\hline 219 & 07174000 & LITTLE CANEY RIVER NEAR COPAN, OK & 36.9709077 & -95.9349807 & 14 & East \\
\hline 220 & 07174200 & $\begin{array}{l}\text { LITTLE CANEY RIVER BLW COTTON CR, } \\
\text { NR COPAN, OK }\end{array}$ & 36.8950786 & -95.9694266 & 21 & East \\
\hline 221 & 07174600 & SAND CREEK AT OKESA, OK & 36.7195235 & -96.1324935 & 33 & East \\
\hline 222 & 07176000 & VERDIGRIS RIVER NEAR CLAREMORE, OK & 36.3075 & -95.6997222 & 13 & East \\
\hline 223 & 07176500 & BIRD CREEK AT AVANT, OK & 36.48508798 & -96.0602743 & 31 & East \\
\hline 224 & 07177000 & HOMINY CREEK NEAR SKIATOOK, OK & 36.348697 & -96.1100024 & 37 & East \\
\hline 225 & 07177500 & BIRD CREEK NEAR SPERRY, OK & 36.27842534 & -95.954162 & 45 & East \\
\hline 226 & 07179500 & NEOSHO R AT COUNCIL GROVE, KS & 38.66584085 & -96.493614 & 25 & East \\
\hline
\end{tabular}


Table 3. Active and inactive U.S. Geological Survey streamgages used for regression analysis within the study area (map numbers 171-254). U.S. Geological Survey streamgage information is from U.S. Geological Survey (2018).-Continued

[no., number; USGS, U.S. Geological Survey; C, Creek; NR, near; KS, Kansas; L, Little; R, River; HWY, Highway; NF, North Fork; AB, above; RE, Reservoir; SF, South Fork; OK, Oklahoma; CLEV, Cleveland; BLW, below; M, Middle; MO, Missouri]

\begin{tabular}{|c|c|c|c|c|c|c|}
\hline $\begin{array}{c}\text { Map no. } \\
\text { (fig. 1) }\end{array}$ & $\begin{array}{c}\text { USGS station } \\
\text { number }\end{array}$ & $\begin{array}{c}\text { USGS station } \\
\text { name }\end{array}$ & Latitude & Longitude & No. annual series & $\begin{array}{l}\text { Hydrologic } \\
\text { region }\end{array}$ \\
\hline 227 & 07180000 & COTTONWOOD R NR MARION, KS & 38.35140376 & -97.0586349 & 29 & East \\
\hline 228 & 07180500 & CEDAR C NR CEDAR POINT, KS & 38.19640618 & -96.8244648 & 78 & East \\
\hline 229 & 07181500 & M CREEK NR ELMDALE, KS & 38.39321704 & -96.7185648 & 11 & East \\
\hline 230 & 07182400 & NEOSHO R AT STRAWN, KS & 38.2698522 & -95.8660774 & 14 & East \\
\hline 231 & 07183000 & NEOSHO R NR IOLA, KS & 37.92225778 & -95.4277596 & 55 & East \\
\hline 232 & 07183100 & OWL C NR PIQUA, KS & 37.8489051 & -95.5742718 & 10 & East \\
\hline 233 & 07183500 & NEOSHO R NR PARSONS, KS & 37.34005866 & -95.1099687 & 40 & East \\
\hline 234 & 07184000 & LIGHTNING C NR MCCUNE, KS & 37.2811711 & -95.0327428 & 78 & East \\
\hline 235 & 07184500 & LABETTE C NR OSWEGO, KS & 37.19379589 & -95.1924979 & 78 & East \\
\hline 236 & 07185000 & NEOSHO RIVER NEAR COMMERCE, OK & 36.92868144 & -94.9574574 & 22 & East \\
\hline 237 & 07185500 & STAHL CREEK NEAR MILLER, MO & 37.19321 & -93.84496 & 25 & East \\
\hline 238 & 07185700 & SPRING RIVER AT LA RUSSELL, MO & 37.15027778 & -94.0613889 & 59 & East \\
\hline 239 & 07185765 & SPRING RIVER AT CARTHAGE, MO & 37.18863889 & -94.3259167 & 50 & East \\
\hline 240 & 07186000 & SPRING RIVER NEAR WACO, MO & 37.2456111 & -94.5664167 & 92 & East \\
\hline 241 & 07186400 & CENTER CREEK NEAR CARTERVILLE, MO & 37.1406145 & -94.3827238 & 28 & East \\
\hline 242 & 07187000 & SHOAL CREEK ABOVE JOPLIN, MO & 37.02316667 & -94.5165556 & 75 & East \\
\hline 243 & 07187500 & SHOAL CREEK NEAR JOPLIN, MO & 37.0347844 & -94.5418911 & 17 & East \\
\hline 244 & 07188000 & SPRING RIVER NEAR QUAPAW, OK & 36.93451148 & -94.7471711 & 45 & East \\
\hline 245 & 07189500 & NEOSHO RIVER NEAR GROVE, OK & 36.6125757 & -94.8238434 & 14 & East \\
\hline 246 & 07191000 & BIG CABIN CREEK NEAR BIG CABIN, OK & 36.56841789 & -95.1521891 & 38 & East \\
\hline 247 & 07191222 & BEATY CREEK NEAR JAY, OK & 36.35535924 & -94.7763388 & 18 & East \\
\hline 248 & 07192000 & PRYOR CREEK NEAR PRYOR, OK & 36.281206 & -95.3258024 & 15 & East \\
\hline 249 & 07232500 & BEAVER RIVER NEAR GUYMON, OK & 36.721415 & -101.4896047 & 39 & West \\
\hline 250 & 07233000 & COLDWATER CREEK NEAR HARDESTY, OK & 36.6439157 & -101.210993 & 24 & West \\
\hline 251 & 07234000 & BEAVER RIVER AT BEAVER, OK & 36.82224819 & -100.5193158 & 41 & West \\
\hline 252 & 07234100 & CLEAR CREEK NEAR ELMWOOD, OK & 36.6450279 & -100.5023688 & 14 & West \\
\hline 253 & 07234500 & BEAVER RIVER NEAR FORT SUPPLY, OK & 36.5917013 & -99.5920598 & 13 & West \\
\hline 254 & 07236000 & WOLF CREEK NEAR FARGO, OK & 36.3992044 & -99.6231707 & 33 & West \\
\hline
\end{tabular}




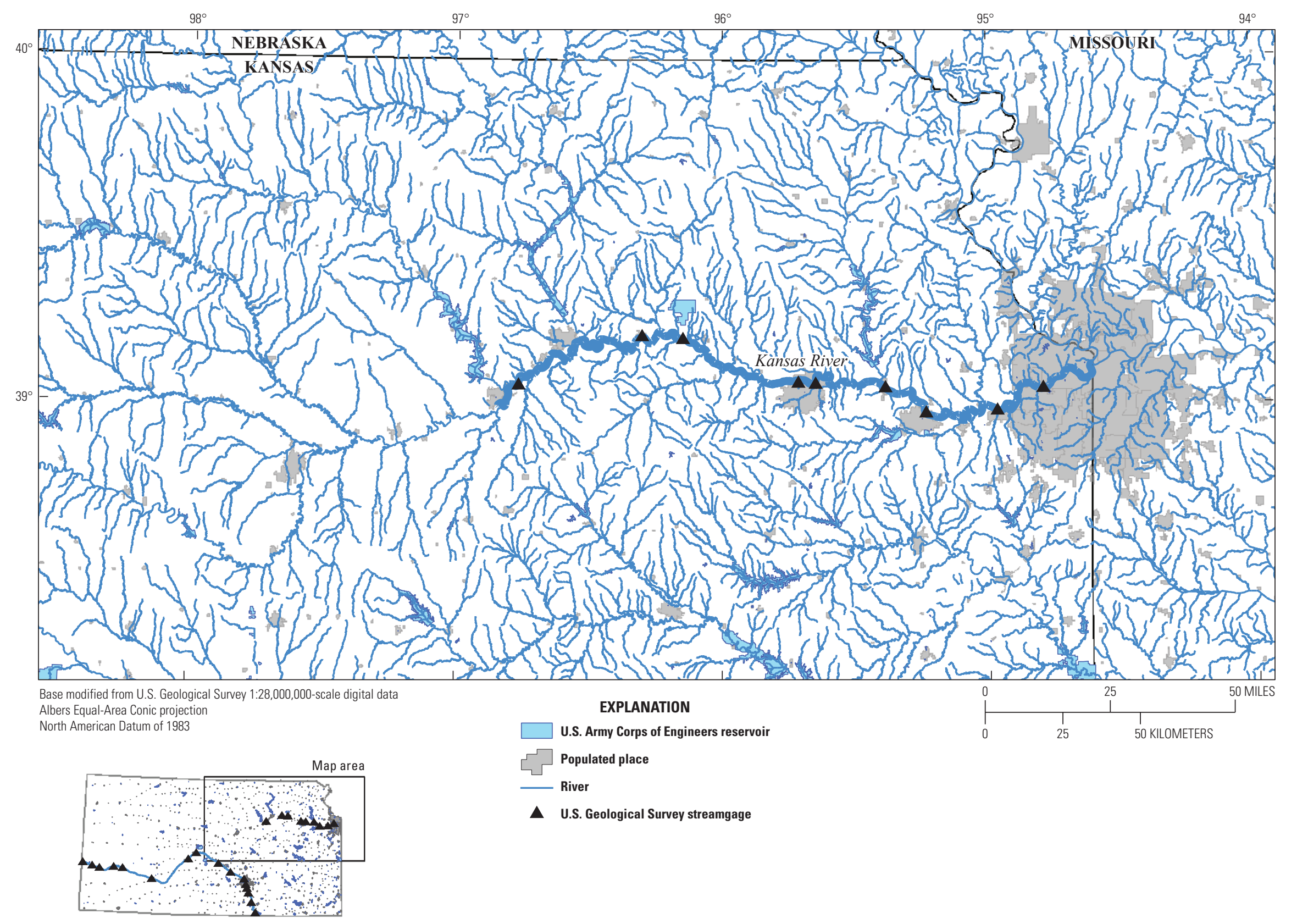

Figure 6. Active Kansas River U.S. Geological Survey streamgages and U.S. Army Corps of Engineers reservoirs that regulate the flow in the Kansas River. 
Table 4. List of active U.S. Geological Survey streamgages on the Kansas and Arkansas Rivers.

[USGS, U.S. Geological Survey; R, River; KS, Kansas; NR, near; HWY, Highway]

\begin{tabular}{cllcc}
\hline USGS station number & \multicolumn{1}{c}{ USGS station name } & Latitude & Longitude & Hydrologic region \\
\hline 06879100 & Kansas R at Fort Riley, KS & 39.06166457 & -96.76639630 & East \\
06887500 & Kansas R at Wamego, KS & 39.19833014 & -96.30555320 & East \\
\hline 06888350 & Kansas R nr Belvue, KS & 39.19305265 & -96.14749450 & East \\
\hline 06888990 & Kansas R at Topeka Water Plant, KS & 39.07194435 & -95.71637150 & East \\
\hline 06889000 & Kansas R at Topeka, KS & 39.06666660 & -95.64970280 & East \\
\hline 06891000 & Kansas R at Lecompton, KS & 39.05111157 & -95.38636330 & East \\
\hline 06891080 & Kansas R at Lawrence, KS & 38.97327778 & -95.23211110 & East \\
\hline 06892350 & Kansas R at De Soto, KS & 38.98333750 & -94.96468930 & East \\
\hline 06892518 & Kansas R nr Lake Quivira, KS & 39.04611110 & -94.78944440 & East \\
\hline 07137500 & Arkansas R nr Coolidge, KS & 38.02751290 & -102.01157060 & West \\
\hline 07138000 & Arkansas R at Syracuse, KS & 37.96612410 & -101.75683870 & West \\
\hline 07138020 & Arkansas R at Kendall, KS & 37.93001500 & -101.54933710 & West \\
\hline 07138070 & Arkansas R at Deerfield, KS & 37.96974420 & -101.12877940 & West \\
07139000 & Arkansas R at Garden City, KS & 37.95585650 & -100.87738440 & West \\
\hline 07139500 & Arkansas R at Dodge City, KS & 37.74474270 & -100.03291260 & West \\
07141220 & Arkansas R nr Larned, KS & 38.20362484 & -99.00231980 & West \\
\hline 07141300 & Arkansas R at Great Bend, KS & 38.35306787 & -98.76425190 & West \\
\hline 07142680 & Arkansas R nr Nickerson, KS & 38.14501130 & -98.11116900 & East \\
\hline 07143330 & Arkansas R nr Hutchinson, KS & 37.94640028 & -97.77504880 & East \\
\hline 07143375 & Arkansas R nr Maize, KS & 37.78140109 & -97.38976650 & East \\
\hline 07144300 & Arkansas R at Wichita, KS & 37.64334849 & -97.33532270 & East \\
\hline 07144550 & Arkansas R at Derby, KS & 37.54418457 & -97.27559990 & East \\
\hline 07144570 & Arkansas R at Mulvane, KS & 37.47550278 & -97.26127220 & East \\
\hline 07145600 & Arkansas R on HWY 160 at Oxford, KS & 37.27440556 & -97.16226390 & East \\
\hline 07146500 & Arkansas R at Arkansas City, KS & 37.03750830 & -97.03921940 & East \\
\hline
\end{tabular}

of the Arkansas River (Juracek and others, 2017). Data for selected low-flow and flow-duration statistics were included from these streamgages for completeness (table 5). The final dataset to develop the regression equations consisted of 254 streamgages from within Kansas and a 50-mi buffer of the neighboring States of Nebraska, Missouri, Oklahoma, and Colorado.

\section{Basin Characteristics}

"Low-flow characteristics of streams are related to the physical, geologic, and climatic properties of drainage basins" (Eash and Barnes, 2017, p. 13). In many studies, drainage area is an important variable in explaining low-flow variability (Kroll and others, 2004; Funkhouser and others, 2008; Eash and Barnes, 2017). Basin characteristics investigated in this study as possible independent variables in the regression analysis were chosen based on their theoretic relation to low flows, findings of previous studies in similar hydrologic areas, and the capability to compute the basin characteristics using geographic-information-system (GIS) technology and digital datasets. "The use of GIS enables the automation of the basin characteristic measurements and solution of the regional regression equations using StreamStats" (Eash and Barnes, 2017, p. 13).

Using GIS technology and other sources, 13 basin characteristics were measured for each of the 254 streamgages used in the regression analysis for this report. A brief explanation of each basin characteristic and the data source used to measure it are included in table 6 .

\section{Basic Statistics}

Daily mean streamflow data were downloaded from the USGS NWIS database using the USGS Surface-Water Toolbox (SWToolbox) version 1.0.4 software (Kiang and 
Table 5. Annual low-flow frequency statistics for active U.S. Geological Survey streamgages on the Kansas and Arkansas Rivers.

[USGS, U.S. Geological Survey; KS, Kansas; nr, near; HWY, Highway]

\begin{tabular}{|c|c|c|c|c|c|c|}
\hline \multirow{2}{*}{$\begin{array}{l}\text { USGS station } \\
\text { number }\end{array}$} & \multirow{2}{*}{ USGS station name } & \multirow{2}{*}{$\begin{array}{l}\text { Parameter } \\
\text { (days) }\end{array}$} & \multicolumn{4}{|c|}{ Recurrence interval (years) } \\
\hline & & & 50 & 20 & 10 & 5 \\
\hline 06879100 & Kansas River at Fort Riley, KS & 1 & 122 & 141 & 164 & 202 \\
\hline 06879100 & Kansas River at Fort Riley, KS & 7 & 132 & 154 & 180 & 221 \\
\hline 06879100 & Kansas River at Fort Riley, KS & 30 & 174 & 196 & 224 & 269 \\
\hline 06887500 & Kansas River at Wamego, KS & 1 & 205 & 267 & 336 & 442 \\
\hline 06887500 & Kansas River at Wamego, KS & 7 & 262 & 327 & 399 & 507 \\
\hline 06887500 & Kansas River at Wamego, KS & 30 & 306 & 383 & 469 & 601 \\
\hline 06888350 & Kansas River nr Belvue, KS & 1 & 401 & 441 & 489 & 569 \\
\hline 06888350 & Kansas River nr Belvue, KS & 7 & 446 & 486 & 534 & 616 \\
\hline 06888350 & Kansas River nr Belvue, KS & 30 & 491 & 546 & 611 & 719 \\
\hline 06888990 & Kansas River at Topeka Water Plant, KS & 1 & 706 & 772 & 831 & 902 \\
\hline 06888990 & Kansas River at Topeka Water Plant, KS & 7 & 882 & 918 & 952 & 994 \\
\hline 06888990 & Kansas River at Topeka Water Plant, KS & 30 & 979 & 993 & 1,010 & 1,050 \\
\hline 06889000 & Kansas River at Topeka, KS & 1 & 235 & 307 & 388 & 512 \\
\hline 06889000 & Kansas River at Topeka, KS & 7 & 280 & 358 & 445 & 578 \\
\hline 06889000 & Kansas River at Topeka, KS & 30 & 328 & 421 & 525 & 685 \\
\hline 06891000 & Kansas River at Lecompton, KS & 1 & 268 & 348 & 439 & 580 \\
\hline 06891000 & Kansas River at Lecompton, KS & 7 & 302 & 391 & 491 & 647 \\
\hline 06888350 & Kansas River at Lecompton, KS & 30 & 380 & 481 & 595 & 773 \\
\hline 06888990 & Kansas River at Lawrence, KS & 1 & 662 & 674 & 690 & 720 \\
\hline 06891080 & Kansas River at Lawrence, KS & 7 & 812 & 838 & 865 & 903 \\
\hline 06891080 & Kansas River at Lawrence, KS & 30 & 829 & 854 & 888 & 948 \\
\hline 06892350 & Kansas River at De Soto, KS & 1 & 281 & 365 & 458 & 602 \\
\hline 06892350 & Kansas River at De Soto, KS & 7 & 339 & 435 & 541 & 703 \\
\hline 06892350 & Kansas River at De Soto, KS & 30 & 430 & 541 & 665 & 856 \\
\hline 06892518 & Kansas River nr Lake Quivira, KS & 1 & 1,110 & 1,130 & 1,150 & 1,190 \\
\hline 06892518 & Kansas River nr Lake Quivira, KS & 7 & 1,180 & 1,190 & 1,210 & 1,240 \\
\hline 06892518 & Kansas River nr Lake Quivira, KS & 30 & 1,190 & 1,260 & 1,320 & 1,410 \\
\hline 07137500 & Arkansas River nr Coolidge, KS & 1 & 0 & 0.197 & 0.768 & 2.38 \\
\hline 07137500 & Arkansas River nr Coolidge, KS & 7 & 0 & 0.356 & 1.23 & 3.49 \\
\hline 07137500 & Arkansas River nr Coolidge, KS & 30 & 0.402 & 1.11 & 2.57 & 6.52 \\
\hline 07138000 & Arkansas River at Syracuse, KS & 1 & 0.047 & 0.224 & 0.623 & 1.83 \\
\hline 07138000 & Arkansas River at Syracuse, KS & 7 & 0.065 & 0.291 & 0.787 & 2.26 \\
\hline 07138000 & Arkansas River at Syracuse, KS & 30 & 0.133 & 0.457 & 1.25 & 3.81 \\
\hline 07138020 & Arkansas River at Kendall, KS & 1 & 0 & 0 & 0 & 0.038 \\
\hline 07138020 & Arkansas River at Kendall, KS & 7 & 0 & 0 & 0 & 0.326 \\
\hline 07138020 & Arkansas River at Kendall, KS & 30 & 0 & 0 & 0 & 1.32 \\
\hline 07138070 & Arkansas River at Deerfield, KS & 1 & 0 & 0 & 0 & 0 \\
\hline 07138070 & Arkansas River at Deerfield, KS & 7 & 0 & 0 & 0 & 0 \\
\hline 07138070 & Arkansas River at Deerfield, KS & 30 & 0 & 0 & 0 & 0 \\
\hline 07139000 & Arkansas River at Garden City, KS & 1 & 0 & 0 & 0 & 0 \\
\hline 07139000 & Arkansas River at Garden City, KS & 7 & 0 & 0 & 0 & 0 \\
\hline 07139000 & Arkansas River at Garden City, KS & 30 & 0 & 0 & 0 & 0 \\
\hline
\end{tabular}


Table 5. Annual low-flow frequency statistics for active U.S. Geological Survey streamgages on the Kansas and Arkansas Rivers.Continued

[USGS, U.S. Geological Survey; KS, Kansas; nr, near; HWY, Highway]

\begin{tabular}{|c|c|c|c|c|c|c|}
\hline \multirow{2}{*}{$\begin{array}{l}\text { USGS station } \\
\text { number }\end{array}$} & \multirow{2}{*}{ USGS station name } & \multirow{2}{*}{$\begin{array}{l}\text { Parameter } \\
\text { (days) }\end{array}$} & \multicolumn{4}{|c|}{ Recurrence interval (years) } \\
\hline & & & 50 & 20 & 10 & 5 \\
\hline 07139500 & Arkansas River at Dodge City, KS & 1 & 0 & 0 & 0 & 0 \\
\hline 07139500 & Arkansas River at Dodge City, KS & 7 & 0 & 0 & 0 & 0 \\
\hline 07139500 & Arkansas River at Dodge City, KS & 30 & 0 & 0 & 0 & 0 \\
\hline 07141220 & Arkansas River nr Larned, KS & 1 & 0 & 0 & 0 & 0 \\
\hline 07141220 & Arkansas River nr Larned, KS & 7 & 0 & 0 & 0 & 0 \\
\hline 07141220 & Arkansas River nr Larned, KS & 30 & 0 & 0 & 0 & 0 \\
\hline 07141300 & Arkansas River at Great Bend, KS & 1 & 0 & 0 & 0.01 & 0.528 \\
\hline 07141300 & Arkansas River at Great Bend, KS & 7 & 0 & 0 & 0.084 & 0.75 \\
\hline 07141300 & Arkansas River at Great Bend, KS & 30 & 0.005 & 0.066 & 0.277 & 1.15 \\
\hline 07142680 & Arkansas River nr Nickerson, KS & 1 & 14.5 & 19 & 24.3 & 32.8 \\
\hline 07142680 & Arkansas River nr Nickerson, KS & 7 & 14.8 & 19.6 & 25.1 & 34.2 \\
\hline 07142680 & Arkansas River nr Nickerson, KS & 30 & 16 & 21.4 & 27.7 & 37.9 \\
\hline 07143330 & Arkansas River nr Hutchinson, KS & 1 & 24.6 & 32 & 40.4 & 53.3 \\
\hline 07143330 & Arkansas River nr Hutchinson, KS & 7 & 27.1 & 35.4 & 44.7 & 59 \\
\hline 07143330 & Arkansas River nr Hutchinson, KS & 30 & 32.2 & 42.2 & 53.4 & 70.6 \\
\hline 07143375 & Arkansas River nr Maize, KS & 1 & 0.516 & 2.22 & 6.69 & 20.2 \\
\hline 07143375 & Arkansas River nr Maize, KS & 7 & 0.72 & 2.91 & 8.4 & 24.3 \\
\hline 07143375 & Arkansas River nr Maize, KS & 30 & 1.46 & 5.17 & 13.5 & 34.9 \\
\hline 07144300 & Arkansas River at Wichita, KS & 1 & 8.41 & 15.3 & 25 & 43.1 \\
\hline 07144300 & Arkansas River at Wichita, KS & 7 & 10.7 & 19.1 & 30.9 & 52.7 \\
\hline 07144300 & Arkansas River at Wichita, KS & 30 & 17.9 & 29.9 & 45.9 & 74.1 \\
\hline 07144550 & Arkansas River at Derby, KS & 1 & 69.7 & 83.2 & 97.3 & 118 \\
\hline 07144550 & Arkansas River at Derby, KS & 7 & 76.3 & 92.2 & 109 & 133 \\
\hline 07144550 & Arkansas River at Derby, KS & 30 & 90.8 & 112 & 134 & 167 \\
\hline 07144570 & Arkansas River at Mulvane, KS & 1 & 68.9 & 91.3 & 114 & 145 \\
\hline 07144570 & Arkansas River at Mulvane, KS & 7 & 152 & 164 & 176 & 191 \\
\hline 07144570 & Arkansas River at Mulvane, KS & 30 & 205 & 225 & 244 & 270 \\
\hline 07145600 & Arkansas River on HWY 160 at Oxford, KS & 1 & 218 & 230 & 243 & 264 \\
\hline 07145600 & Arkansas River on HWY 160 at Oxford, KS & 7 & 221 & 240 & 260 & 287 \\
\hline 07145600 & Arkansas River on HWY 160 at Oxford, KS & 30 & 338 & 345 & 356 & 377 \\
\hline 07146500 & Arkansas River at Arkansas City, KS & 1 & 23.8 & 43.3 & 70 & 117 \\
\hline 07146500 & Arkansas River at Arkansas City, KS & 7 & 30.7 & 53.4 & 83.6 & 137 \\
\hline 07146500 & Arkansas River at Arkansas City, KS & 30 & 53 & 83.2 & 121 & 185 \\
\hline
\end{tabular}

others, 2018; U.S. Geological Survey, 2018). SWToolbox is a Windows-based desktop computer application that provides a set of procedures for statistical analysis of streamflow time series, which includes the computation of low-flow frequency statistics (Kiang and others, 2018). It combines the functionality of the USGS SWSTAT and the U.S. Environmental
Protection Agency DFLOW software programs (Kiang and others, 2018). The basic statistics for all the streamgages mentioned in this study were computed using a utility within SWToolbox. The minimum, maximum, mean, standard deviation, and count of all daily mean streamflows are provided in tables 7-8. 
Table 6. Basin characteristics tested for significance during the regression analysis.

[E, denotes exponentiation; NA, not applicable]

\begin{tabular}{|c|c|c|c|c|}
\hline $\begin{array}{l}\text { Basin characteristic and } \\
\text { abbreviation }\end{array}$ & Unit & Source & Range & Website \\
\hline $\begin{array}{l}\text { Contributing drainage } \\
\text { area-CONTDA }\end{array}$ & Square miles & U.S. Geological Survey, 2018 & 3.86 to 24,900 & https://doi.org/10.5066/F7P55KJN \\
\hline $\begin{array}{l}\text { Mean annual precipita- } \\
\text { tion for } 1895-2017- \\
\text { PRECIPfull }\end{array}$ & Inches & PRISM Climate Group, 2020 & 14.6 to 44.4 & https://www.prism.oregonstate.edu/explorer/ \\
\hline $\begin{array}{l}\text { Mean annual precipitation } \\
\text { for 1961-90-PRECIP } \\
1961-90\end{array}$ & Inches & PRISM Climate Group, 2020 & 13.7 to 44.4 & https://www.prism.oregonstate.edu/ \\
\hline $\begin{array}{l}\text { Mean annual precipitation } \\
\text { for 1971-2000-PRECIP } \\
\text { 1971-2000 }\end{array}$ & Inches & PRISM Climate Group, 2020 & 14.6 to 46.4 & https://www.prism.oregonstate.edu/ \\
\hline $\begin{array}{l}\text { Mean annual precipitation } \\
\text { for 1981-2010-PRECIP } \\
\text { 1981-2010 }\end{array}$ & Inches & PRISM Climate Group, 2020 & 14.9 to 47.0 & https://www.prism.oregonstate.edu/ \\
\hline $\begin{array}{l}\text { Saturated hydraulic } \\
\text { conductivity-KSAT }\end{array}$ & $\begin{array}{l}\text { Micrometers } \\
\text { per second }\end{array}$ & Wieczorek, 2014 & 1.21 to 89.3 & https://doi.org/10.3133/tm3B10 \\
\hline $\begin{array}{l}\text { Drainage basin slope- } \\
\text { SLOPE }\end{array}$ & Percent & $\begin{array}{l}\text { ArcGIS slope tool on National } \\
\text { Elevation Dataset }\end{array}$ & 0.82 to 12.2 & NA \\
\hline Base-flow index-BFI & Dimensionless & Barlow and others, 2015 & $4.00 \mathrm{E}-03$ to 0.82 & $\begin{array}{l}\text { https://www.usgs.gov/software/groundwater-toolbox-a-graphical-and-mapping- } \\
\text { interface-analysis-hydrologic-data }\end{array}$ \\
\hline $\begin{array}{l}\text { Reservoir storage- } \\
\text { STORAGE }\end{array}$ & Percent & U.S. Geological Survey, 2006 & 0.02 to 9.25 & $\begin{array}{l}\text { https://www.usgs.gov/centers/eros/science/national-land-cover-database?qt- } \\
\text { science_center_objects=0\#qt-science_center_objects }\end{array}$ \\
\hline Forest land cover-FOREST & Percent & U.S. Geological Survey, 2006 & $1.00 \mathrm{E}-03$ to 35.5 & $\begin{array}{l}\text { https://www.usgs.gov/centers/eros/science/national-land-cover-database?qt- } \\
\text { science_center_objects=0\#qt-science_center_objects }\end{array}$ \\
\hline $\begin{array}{l}\text { Shrub land cover- } \\
\text { LC06SHRUB }\end{array}$ & Percent & U.S. Geological Survey, 2006 & $6.42 \mathrm{E}-05$ to 35.8 & $\begin{array}{l}\text { https://www.usgs.gov/centers/eros/science/national-land-cover-database?qt- } \\
\text { science_center_objects=0\#qt-science_center_objects }\end{array}$ \\
\hline $\begin{array}{l}\text { Pasture land cover- } \\
\text { LC06PAST }\end{array}$ & Percent & U.S. Geological Survey, 2006 & $3.37 \mathrm{E}-03$ to 83.7 & $\begin{array}{l}\text { https://www.usgs.gov/centers/eros/science/national-land-cover-database?qt- } \\
\text { science_center_objects=0\#qt-science_center_objects }\end{array}$ \\
\hline $\begin{array}{l}\text { Crop land cover- } \\
\text { LC06CROP }\end{array}$ & Percent & U.S. Geological Survey, 2006 & 0.17 to 85.9 & $\begin{array}{l}\text { https://www.usgs.gov/centers/eros/science/national-land-cover-database?qt- } \\
\text { science_center_objects=0\#qt-science_center_objects }\end{array}$ \\
\hline
\end{tabular}


Table 7. Basic statistics for daily mean streamflows of U.S. Geological Survey streamgages used in the regression analysis (map numbers 1-126), computed using SWToolbox (Kiang and others, 2018).

[no., number; USGS, U.S. Geological Survey; NE, Nebraska; MO, Missouri; C, Creek; NR, near; KS, Kansas; CO, Colorado; R, River; SF, South Fork; ST, State; AB, above; NF, North Fork; BL, below; RE, Reservoir; L, Little; ST., Saint; ST, Street; BLVD, Boulevard; EXT, Exit; KC, Kansas City]

\begin{tabular}{|c|c|c|c|c|c|c|c|}
\hline $\begin{array}{c}\text { Map no. } \\
\text { (fig. 1) }\end{array}$ & USGS station number & USGS station name & Minimum & Maximum & Mean & $\begin{array}{l}\text { Standard } \\
\text { deviation }\end{array}$ & Count \\
\hline 1 & 06767500 & PLUM CREEK NEAR SMITHFIELD, NE & 0 & 1,850 & 9.67 & 47.931 & 7,120 \\
\hline 2 & 06768020 & SPRING CREEK NEAR OVERTON, NE & 0 & 464 & 26.1 & 35.879 & 7,670 \\
\hline 3 & 06769525 & ELM CREEK NEAR ELM CREEK, NE & 0 & 893 & 4.1 & 20.911 & 7,670 \\
\hline 4 & 06810500 & LITTLE NEMAHA RIVER NEAR SYRACUSE, NE & 0 & 14,500 & 63.4 & 336.37 & 6,209 \\
\hline 5 & 06811500 & LITTLE NEMAHA RIVER AT AUBURN, NE & 0.87 & 70,400 & 325 & $1,337.90$ & 24,472 \\
\hline 6 & 06813000 & TARKIO RIVER AT FAIRFAX, MO & 0 & 11,100 & 235 & 609.92 & 28,677 \\
\hline 7 & 06814000 & TURKEY C NR SENECA, KS & 0 & 16,700 & 123 & 514.85 & 24,837 \\
\hline 8 & 06814500 & NORTH FORK BIG NEMAHA RIVER AT HUMBOLDT, NE & 0.07 & 30,000 & 214 & 950.76 & 16,985 \\
\hline 9 & 06815000 & BIG NEMAHA RIVER AT FALLS CITY, NE & 0.69 & 57,600 & 599 & $2,029.50$ & 26,663 \\
\hline 10 & 06815500 & MUDDY CREEK AT VERDON, NE & 1 & 14,300 & 67.2 & 303.15 & 6,940 \\
\hline 11 & 06817500 & NODAWAY RIVER NEAR BURLINGTON JUNCTION, MO & 1.1 & 34,500 & 589 & $1,476.80$ & 23,116 \\
\hline 12 & 06817700 & NODAWAY RIVER NEAR GRAHAM, MO & 22 & 52,000 & 1,010 & $2,240.20$ & 12,419 \\
\hline 13 & 06818900 & PLATTE RIVER AT RAVENWOOD, MO & 2.4 & 9,950 & 268 & 805.92 & 4,383 \\
\hline 14 & 06819500 & ONE HUNDRED AND TWO RIVER AT MARYVILLE, MO & 0 & 25,500 & 262 & 890.07 & 26,947 \\
\hline 15 & 06820500 & PLATTE RIVER NEAR AGENCY, MO & 0 & 57,500 & 1,030 & 2,710 & 32,961 \\
\hline 16 & 06821080 & LITTLE PLATTE RIVER NEAR PLATTSBURG, MO & 0 & 7,730 & 47.2 & 247.03 & 5,854 \\
\hline 17 & 06821150 & LITTLE PLATTE RIVER AT SMITHVILLE, MO & 0 & 21,100 & 161 & 683.08 & 3,653 \\
\hline 18 & 06821190 & PLATTE RIVER AT SHARPS STATION, MO & 12 & 41,200 & 1,760 & $3,271.40$ & 13,880 \\
\hline 19 & 06823500 & BUFFALO CREEK NEAR HAIGLER, NE & 0 & 90 & 5.83 & 4.0813 & 27,759 \\
\hline 20 & 06825000 & SOUTH FORK REPUBLICAN RIVER NEAR IDALIA, CO & 0 & 6,640 & 32.8 & 120.83 & 7,305 \\
\hline 21 & 06825500 & LANDSMAN CREEK NEAR HALE, CO & 0 & 1,800 & 3.77 & 36.73 & 9,132 \\
\hline 22 & 06827000 & SF REPUBLICAN R NR CO-KS ST LINE, KS & 0 & 2,000 & 20.2 & 46.517 & 9,253 \\
\hline 23 & 06827500 & SOUTH FORK REPUBLICAN RIVER NEAR BENKELMAN, NE & 0 & 5,500 & 73 & 208.38 & 4,383 \\
\hline 24 & 06828000 & REPUBLICAN RIVER AT MAX, NE & 0 & 85,000 & 191 & $1,256.10$ & 5,479 \\
\hline 25 & 06835000 & STINKING WATER CREEK NEAR PALISADE, NE & 4.9 & 1,640 & 37.1 & 34.473 & 16,071 \\
\hline 26 & 06836000 & BLACKWOOD CREEK NEAR CULBERTSON, NE & 0 & 1,270 & 5.89 & 28.804 & 14,245 \\
\hline 27 & 06836500 & DRIFTWOOD CREEK NEAR MCCOOK, NE & 0 & 3,950 & 8.05 & 54.122 & 25,933 \\
\hline 28 & 06837300 & RED WILLOW CREEK ABOVE HUGH BUTLER LAKE, NE & 3.1 & 734 & 26.2 & 24.257 & 12,053 \\
\hline 29 & 06838000 & RED WILLOW CREEK NEAR RED WILLOW, NE & 2 & 5,730 & 42.9 & 107.77 & 7,670 \\
\hline 30 & 06840000 & FOX CREEK AT CURTIS, NE & 0.71 & 1,560 & 7.05 & 22.133 & 7,669 \\
\hline
\end{tabular}


Table 7. Basic statistics for daily mean streamflows of U.S. Geological Survey streamgages used in the regression analysis (map numbers 1-126), computed using SWToolbox (Kiang and others, 2018).-Continued

[no., number; USGS, U.S. Geological Survey; NE, Nebraska; MO, Missouri; C, Creek; NR, near; KS, Kansas; CO, Colorado; R, River; SF, South Fork; ST, State; AB, above; NF, North Fork; BL, below; RE, Reservoir; L, Little; ST., Saint; ST, Street; BLVD, Boulevard; EXT, Exit; KC, Kansas City]

\begin{tabular}{|c|c|c|c|c|c|c|c|}
\hline $\begin{array}{c}\text { Map no. } \\
\text { (fig. 1) }\end{array}$ & USGS station number & USGS station name & Minimum & Maximum & Mean & $\begin{array}{l}\text { Standard } \\
\text { deviation }\end{array}$ & Count \\
\hline 31 & 06841000 & MEDICINE CREEK ABOVE HARRY STRUNK LAKE, NE & 9.1 & 5,900 & 62.6 & 115 & 16,071 \\
\hline 32 & 06843000 & MEDICINE CREEK AT CAMBRIDGE, NE & 6 & 28,500 & 93.1 & 513.56 & 4,047 \\
\hline 33 & 06844000 & MUDDY CREEK AT ARAPAHOE, NE & 0 & 4,570 & 14.8 & 81.188 & 13,514 \\
\hline 34 & 06844210 & TURKEY CREEK AT EDISON, NE & 0.01 & 330 & 7.61 & 13.002 & 5,479 \\
\hline 35 & 06844900 & SF SAPPA C NR ACHILLES, KS & 0 & 3,060 & 2.48 & 35.171 & 17,897 \\
\hline 36 & 06845000 & SAPPA C NR OBERLIN, KS & 0 & 5,100 & 14.2 & 114.03 & 15,350 \\
\hline 37 & 06845110 & SAPPA C NR LYLE, KS & 0 & 877 & 7.12 & 23.149 & 7,670 \\
\hline 38 & 06845200 & SAPPA CREEK NEAR BEAVER CITY, NE & 0 & 4,700 & 38 & 157.76 & 12,875 \\
\hline 39 & 06846000 & BEAVER C AT LUDELL, KS & 0 & 2,000 & 9.41 & 58.043 & 9,404 \\
\hline 40 & 06846500 & BEAVER C AT CEDAR BLUFFS, KS & 0 & 4,560 & 10.1 & 74.087 & 25,568 \\
\hline 41 & 06847000 & BEAVER CREEK NEAR BEAVER CITY, NE & 0 & 5,130 & 19 & 87.125 & 21,549 \\
\hline 42 & 06847500 & SAPPA CREEK NEAR STAMFORD, NE & 0 & 16,600 & 37 & 184.64 & 25,933 \\
\hline 43 & 06847900 & PRAIRIE DOG C AB KEITH SEBELIUS LAKE, KS & 0 & 3,150 & 7.61 & 55.974 & 19,724 \\
\hline 44 & 06848000 & PRAIRIE DOG C AT NORTON, KS & 0 & 9,650 & 42.4 & 258.7 & 6,939 \\
\hline 45 & 06848500 & PRAIRIE DOG C NR WOODRUFF, KS & 0 & 9,700 & 57.5 & 291.15 & 7,721 \\
\hline 46 & 06850500 & REPUBLICAN RIVER NEAR BLOOMINGTON, NE & 6.8 & 116,000 & 720 & $2,111.50$ & 7,305 \\
\hline 47 & 06851000 & CENTER CREEK AT FRANKLIN, NE & 0 & 839 & 8.04 & 20.663 & 11,320 \\
\hline 48 & 06851500 & THOMPSON CREEK AT RIVERTON, NE & 8.1 & 3,230 & 32.3 & 91.766 & 11,687 \\
\hline 49 & 06853500 & REPUBLICAN R NR HARDY, NE & 0 & 117,000 & 867 & $2,057.30$ & 10,190 \\
\hline 50 & 06853800 & WHITE ROCK C NR BURR OAK, KS & 0 & 6,000 & 26.2 & 134.98 & 21,550 \\
\hline 51 & 06854500 & REPUBLICAN R AT SCANDIA, KS & 0 & 115,000 & 827 & $1,988.90$ & 7,992 \\
\hline 52 & 06855800 & BUFFALO C NR JAMESTOWN, KS & 0 & 19,900 & 70.2 & 342.39 & 10,957 \\
\hline 53 & 06855900 & WOLF C NR CONCORDIA, KS & 0 & 2,320 & 10.9 & 58.977 & 6,940 \\
\hline 54 & 06856600 & REPUBLICAN R AT CLAY CENTER, KS & 1 & 103,000 & 1,140 & 2,244 & 12,053 \\
\hline 55 & 06857000 & REPUBLICAN R AT MILFORD, KS & 57 & 71,000 & 1,610 & $3,800.70$ & 4,414 \\
\hline 56 & 06858500 & NF SMOKY HILL R NR MCALLASTER, KS & 0 & 2,360 & 3.67 & 45.371 & 11,415 \\
\hline 57 & 06859500 & LADDER C BL CHALK C NR SCOTT CITY, KS & 0 & 6,580 & 11.6 & 115.66 & 10,227 \\
\hline 58 & 06860000 & SMOKY HILL R AT ELKADER, KS & 0 & 13,700 & 19.3 & 193.21 & 28,124 \\
\hline 59 & 06861000 & SMOKY HILL R NR ARNOLD, KS & 0 & 14,200 & 38.5 & 307.41 & 24,472 \\
\hline 60 & 06862500 & SMOKY HILL R NR ELLIS, KS & 0 & 8,960 & 133 & 461.45 & 3,862 \\
\hline
\end{tabular}


Table 7. Basic statistics for daily mean streamflows of U.S. Geological Survey streamgages used in the regression analysis (map numbers 1-126), computed using SWToolbox (Kiang and others, 2018).-Continued

[no., number; USGS, U.S. Geological Survey; NE, Nebraska; MO, Missouri; C, Creek; NR, near; KS, Kansas; CO, Colorado; R, River; SF, South Fork; ST, State; AB, above; NF, North Fork; BL, below; RE, Reservoir; L, Little; ST., Saint; ST, Street; BLVD, Boulevard; EXT, Exit; KC, Kansas City]

\begin{tabular}{|c|c|c|c|c|c|c|c|}
\hline $\begin{array}{c}\text { Map no. } \\
\text { (fig. 1) }\end{array}$ & USGS station number & USGS station name & Minimum & Maximum & Mean & $\begin{array}{l}\text { Standard } \\
\text { deviation }\end{array}$ & Count \\
\hline 61 & 06863300 & BIG C NR OGALLAH, KS & 0 & 6,720 & 22.7 & 166.86 & 4,383 \\
\hline 62 & 06863500 & BIG C NR HAYS, KS & 0 & 10,600 & 28 & 179.97 & 25,933 \\
\hline 63 & 06863900 & NF BIG C NR VICTORIA, KS & 0 & 4,380 & 3.33 & 56.222 & 9,131 \\
\hline 64 & 06864000 & SMOKY HILL R NR RUSSELL, KS & 0 & 12,500 & 189 & 662.26 & 3,652 \\
\hline 65 & 06864500 & SMOKY HILL R AT ELLSWORTH, KS & 1 & 41,800 & 212 & 794.35 & 13,952 \\
\hline 66 & 06866000 & SMOKY HILL R AT LINDSBORG, KS & 0 & 24,000 & 241 & 764.67 & 9,693 \\
\hline 67 & 06867000 & SALINE R NR RUSSELL, KS & 0.06 & 23,400 & 85.5 & 391.59 & 23,863 \\
\hline 68 & 06867500 & PARADISE C NR PARADISE, KS & 0 & 8,350 & 18.4 & 147.84 & 8,075 \\
\hline 69 & 06868000 & SALINE R NR WILSON, KS & 2 & 16,200 & 169 & 623.92 & 12,053 \\
\hline 70 & 06868400 & WOLF C NR LUCAS, KS & 0.13 & 3,090 & 14.9 & 99.334 & 4,017 \\
\hline 71 & 06869500 & SALINE R AT TESCOTT, KS & 0 & 47,600 & 250 & 881.41 & 15,660 \\
\hline 72 & 06869950 & MULBERRY C NR SALINA, KS & 0 & 15,400 & 32.5 & 316.7 & 5,479 \\
\hline 73 & 06871000 & NF SOLOMON R AT GLADE, KS & 0 & 10,900 & 25.6 & 152.84 & 23,376 \\
\hline 74 & 06871500 & BOW C NR STOCKTON, KS & 0 & 4,990 & 15.1 & 93.238 & 24,107 \\
\hline 75 & 06871800 & NF SOLOMON R AT KIRWIN, KS & 0 & 12,000 & 85.4 & 390.67 & 7,824 \\
\hline 76 & 06871900 & DEER C NR PHILLIPSBURG, KS & 0 & 1,850 & 4.06 & 47.073 & 5,114 \\
\hline 77 & 06873000 & SF SOLOMON R AB WEBSTER RE, KS & 0 & 35,000 & 47.1 & 365.6 & 26,208 \\
\hline 78 & 06873500 & SF SOLOMON R AT ALTON, KS & 0 & 52,900 & 97.5 & 744.12 & 8,733 \\
\hline 79 & 06873700 & KILL C NR BLOOMINGTON, KS & 0 & 685 & 1.93 & 17.412 & 6,575 \\
\hline 80 & 06876000 & SOLOMON R AT BELOIT, KS & 0 & 113,000 & 456 & $2,156.20$ & 9,131 \\
\hline 81 & 06876700 & SALT C NR ADA, KS & 0 & 10,400 & 61.6 & 276.39 & 20,819 \\
\hline 82 & 06876900 & SOLOMON R AT NILES, KS & 1 & 157,000 & 623 & $2,317.10$ & 15,400 \\
\hline 83 & 06877000 & SMOKY HILL R AT SOLOMON, KS & 25 & 18,400 & 958 & $1,834.60$ & 5,113 \\
\hline 84 & 06877600 & SMOKY HILL R AT ENTERPRISE, KS & 45 & 32,000 & 1,400 & $2,764.40$ & 4,018 \\
\hline 85 & 06878000 & CHAPMAN C NR CHAPMAN, KS & 0.2 & 12,600 & 86 & 350.86 & 23,011 \\
\hline 86 & 06878500 & LYON C NR WOODBINE, KS & 0 & 17,200 & 105 & 529.99 & 7,305 \\
\hline 87 & 06881000 & BIG BLUE RIVER NEAR CRETE, NE & 6 & 21,400 & 391 & 984.61 & 23,011 \\
\hline 88 & 06881200 & TURKEY CREEK NEAR WILBER, NE & 0 & 13,100 & 95.4 & 393.34 & 12,418 \\
\hline 89 & 06881380 & TURKEY CREEK NEAR DEWITT, NE & -11.2 & 21,000 & 133 & 627.73 & 5,114 \\
\hline 90 & 06881500 & BIG BLUE R AT BEATRICE, NE & 5 & 44,400 & 792 & $1,900.70$ & 8,475 \\
\hline
\end{tabular}


Table 7. Basic statistics for daily mean streamflows of U.S. Geological Survey streamgages used in the regression analysis (map numbers 1-126), computed using SWToolbox (Kiang and others, 2018).-Continued

[no., number; USGS, U.S. Geological Survey; NE, Nebraska; MO, Missouri; C, Creek; NR, near; KS, Kansas; CO, Colorado; R, River; SF, South Fork; ST, State; AB, above; NF, North Fork; BL, below; RE, Reservoir; L, Little; ST., Saint; ST, Street; BLVD, Boulevard; EXT, Exit; KC, Kansas City]

\begin{tabular}{|c|c|c|c|c|c|c|c|}
\hline $\begin{array}{c}\text { Map no. } \\
\text { (fig. 1) }\end{array}$ & USGS station number & USGS station name & Minimum & Maximum & Mean & $\begin{array}{l}\text { Standard } \\
\text { deviation }\end{array}$ & Count \\
\hline 91 & 06882000 & BIG BLUE RIVER AT BARNESTON, NE & 1 & 50,000 & 842 & $2,084.60$ & 30,681 \\
\hline 92 & 06882500 & BIG BLUE R AT HULL, KS & 2 & 14,800 & 475 & $1,003.70$ & 6,175 \\
\hline 93 & 06882510 & BIG BLUE R AT MARYSVILLE, KS & 16.1 & 35,000 & 1,020 & $2,252.90$ & 11,688 \\
\hline 94 & 06883000 & LITTLE BLUE RIVER NEAR DEWEESE, NE & 2 & 14,300 & 135 & 414.26 & 22,645 \\
\hline 95 & 06883570 & LITTLE BLUE RIVER NEAR ALEXANDRIA (GILEAD), NE & 2.9 & 16,300 & 234 & 626.3 & 11,119 \\
\hline 96 & 06884000 & LITTLE BLUE RIVER NEAR FAIRBURY, NE & 14 & 43,700 & 365 & $1,110.60$ & 34,150 \\
\hline 97 & 06884025 & LITTLE BLUE RIVER AT HOLLENBERG, KS & 24.2 & 39,300 & 472 & $1,334.70$ & 15,706 \\
\hline 98 & 06884200 & MILL C AT WASHINGTON, KS & 0 & 10,000 & 98.3 & 382.96 & 20,819 \\
\hline 99 & 06884400 & L BLUE R NR BARNES, KS & 21.1 & 46,100 & 653 & $1,657.50$ & 21,185 \\
\hline 100 & 06884500 & L BLUE R AT WATERVILLE, KS & 27 & 45,300 & 621 & $1,734.20$ & 11,652 \\
\hline 101 & 06885500 & BLACK VERMILLION R NR FRANKFORT, KS & 0 & 28,800 & 168 & 716.07 & 23,011 \\
\hline 102 & 06886000 & BIG BLUE R AT RANDOLPH, KS & 42 & 75,000 & 1,670 & $3,975.90$ & 14,976 \\
\hline 103 & 06888000 & VERMILLION C NR WAMEGO, KS & 0 & 13,200 & 83.9 & 373.82 & 15,672 \\
\hline 104 & 06888500 & MILL C NR PAXICO, KS & 0 & 21,700 & 184 & 692.69 & 23,011 \\
\hline 105 & 06889120 & SOLDIER C NR BANCROFT, KS & 0 & 672 & 6.83 & 34.351 & 8,766 \\
\hline 106 & 06889140 & SOLDIER C NR SOLDIER, KS & 0 & 1,920 & 10.6 & 55.544 & 12,418 \\
\hline 107 & 06889160 & SOLDIER C NR CIRCLEVILLE, KS & 0.03 & 5,830 & 32.3 & 155.52 & 13,514 \\
\hline 108 & 06889180 & SOLDIER C NR ST. CLERE, KS & 0.11 & 4,410 & 50 & 199.28 & 6,209 \\
\hline 109 & 06889200 & SOLDIER C NR DELIA, KS & 0 & 24,900 & 92.3 & 412.93 & 21,185 \\
\hline 110 & 06889500 & SOLDIER C NR TOPEKA, KS & 0 & 30,600 & 155 & 657.27 & 30,747 \\
\hline 111 & 06890100 & DELAWARE R NR MUSCOTAH, KS & 0 & 26,000 & 257 & 995.87 & 17,167 \\
\hline 112 & 06890500 & DELAWARE R AT VALLEY FALLS, KS & 0.1 & 55,200 & 383 & $1,598.90$ & 16,071 \\
\hline 113 & 06891260 & WAKARUSA R NR RICHLAND, KS & 0 & 15,800 & 91 & 420.34 & 5,114 \\
\hline 114 & 06891500 & WAKARUSA R NR LAWRENCE, KS & 0 & 22,600 & 192 & 796.07 & 18,628 \\
\hline 115 & 06891810 & STRANGER C NR POTTER, KS & 0.07 & 16,300 & 104 & 486.83 & 4,017 \\
\hline 116 & 06892000 & STRANGER C NR TONGANOXIE, KS & 0 & 23,000 & 248 & 832.52 & 31,777 \\
\hline 117 & 06892360 & KILL C AT 95TH ST NR DESOTO, KS & 0 & 2,450 & 35.1 & 134.61 & 5,114 \\
\hline 118 & 06893080 & BLUE R NR STANLEY, KS & 0 & 5,520 & 36.4 & 169.21 & 15,341 \\
\hline 119 & 06893150 & BLUE RIVER AT BLUE RIDGE BLVD EXT IN KC, MO & 1.1 & 5,920 & 85.7 & 275.36 & 4,863 \\
\hline 120 & 06893300 & INDIAN C AT OVERLAND PARK, KS & 0 & 1,970 & 23.1 & 98.71 & 5,844 \\
\hline
\end{tabular}


Table 7. Basic statistics for daily mean streamflows of U.S. Geological Survey streamgages used in the regression analysis (map numbers 1-126), computed using SWToolbox (Kiang and others, 2018).-Continued

[no., number; USGS, U.S. Geological Survey; NE, Nebraska; MO, Missouri; C, Creek; NR, near; KS, Kansas; CO, Colorado; R, River; SF, South Fork; ST, State; AB, above; NF, North Fork; BL, below; RE, Reservoir; L, Little; ST., Saint; ST, Street; BLVD, Boulevard; EXT, Exit; KC, Kansas City]

\begin{tabular}{|c|c|c|c|c|c|c|c|}
\hline $\begin{array}{c}\text { Map no. } \\
\text { (fig. 1) }\end{array}$ & USGS station number & USGS station name & Minimum & Maximum & Mean & $\begin{array}{l}\text { Standard } \\
\text { deviation }\end{array}$ & Count \\
\hline 121 & 06893500 & BLUE RIVER AT KANSAS CITY, MO & 0 & 20,000 & 177 & 622.98 & 28,124 \\
\hline 122 & 06893578 & BLUE RIVER AT STADIUM DRIVE IN KANSAS CITY, MO & 19.6 & 18,800 & 300 & 830.58 & 5,114 \\
\hline 123 & 06893793 & LITTLE BLUE RIVER BELOW LONGVIEW DAM AT KC, MO & 0 & 3,940 & 39.5 & 163.82 & 6,210 \\
\hline 124 & 06893890 & EAST FORK LITTLE BLUE RIVER NEAR BLUE SPRINGS, MO & 0 & 4,850 & 29.3 & 82.654 & 11,753 \\
\hline 125 & 06894000 & LITTLE BLUE RIVER NEAR LAKE CITY, MO & 0 & 27,700 & 163 & 468.13 & 25,202 \\
\hline 126 & 06894500 & EAST FORK FISHING RIVER AT EXCELSIOR SPRINGS, MO & 0 & 3,890 & 13.2 & 90.581 & 6,940 \\
\hline
\end{tabular}


Table 8. Basic statistics for daily mean streamflows of U.S. Geological Survey streamgages used in the regression analysis (map numbers 127-254), computed using SWToolbox (Kiang and others, 2018).

[no., number; USGS, U.S. Geological Survey; MO, Missouri; R, River; NR, near; KS, Kansas; C, Creek; L, Little; CO, Colorado; HWY, Highway; NF, North Fork; AB, above; RE, Reservoir; SF, South Fork; OK, Oklahoma]

\begin{tabular}{|c|c|c|c|c|c|c|c|}
\hline $\begin{array}{l}\text { Map no. } \\
\text { (fig. 1) }\end{array}$ & USGS station number & USGS station name & Minimum & Maximum & Mean & $\begin{array}{l}\text { Standard } \\
\text { deviation }\end{array}$ & Count \\
\hline 127 & 06895000 & CROOKED RIVER NEAR RICHMOND, MO & 0 & 17,900 & 120 & 488.54 & 11,687 \\
\hline 128 & 06896500 & THOMPSON BRANCH NEAR ALBANY, MO & 0 & 400 & 2.91 & 16.064 & 5,844 \\
\hline 129 & 06897000 & EAST FORK BIG CREEK NEAR BETHANY, MO & 0 & 6,200 & 54.8 & 214.49 & 21,182 \\
\hline 130 & 06897500 & GRAND RIVER NEAR GALLATIN, MO & 2 & 85,500 & 1,310 & $3,913.80$ & 34,699 \\
\hline 131 & 06898100 & THOMPSON RIVER AT MOUNT MORIAH, MO & 8 & 18,100 & 519 & 1,198 & 5,844 \\
\hline 132 & 06899700 & SHOAL CREEK NEAR BRAYMER, MO & 0 & 22,000 & 278 & 859.48 & 10,695 \\
\hline 133 & 06907500 & $\begin{array}{l}\text { SOUTH FORK BLACKWATER RIVER NEAR ELM, } \\
\text { MO }\end{array}$ & 0 & 2,250 & 11.8 & 64.501 & 8,766 \\
\hline 134 & 06910800 & MARAIS DES CYGNES R NR READING, KS & 0 & 25,000 & 103 & 508.97 & 17,167 \\
\hline 135 & 06911000 & MARAIS DES CYGNES R AT MELVERN, KS & 0 & 39,400 & 195 & 925.66 & 11,688 \\
\hline 136 & 06911490 & SALT C AT LYNDON, KS & 0 & 11,800 & 52.4 & 316.63 & 6,209 \\
\hline 137 & 06911500 & SALT C NR LYNDON, KS & 0 & 17,600 & 65.4 & 338.76 & 21,549 \\
\hline 138 & 06911900 & DRAGOON C NR BURLINGAME, KS & 0 & 14,800 & 65.5 & 350.27 & 20,819 \\
\hline 139 & 06912500 & HUNDRED AND TEN MILE C NR QUENEMO, KS & 0 & 27,700 & 182 & 899.2 & 8,400 \\
\hline 140 & 06913000 & MARAIS DES CYGNES R NR POMONA, KS & 0 & 29,500 & 328 & $1,434.50$ & 5,114 \\
\hline 141 & 06913500 & MARAIS DES CYGNES R NR OTTAWA, KS & 0 & 134,000 & 621 & $2,539.10$ & 17,172 \\
\hline 142 & 06914000 & POTTAWATOMIE C NR GARNETT, KS & 0 & 33,400 & 236 & 979.46 & 22,462 \\
\hline 143 & 06914100 & POTTAWATOMIE C NR SCIPIO, KS & 0 & 58,700 & 234 & 1,222 & 5,479 \\
\hline 144 & 06914950 & BIG BULL C NR EDGERTON, KS & 0 & 2,520 & 21.2 & 106.91 & 8,401 \\
\hline 145 & 06914990 & L BULL C NR SPRING HILL, KS & 0 & 930 & 8.87 & 38.146 & 8,401 \\
\hline 146 & 06915000 & BIG BULL C NR HILLSDALE, KS & 0 & 18,000 & 96.6 & 501.72 & 7,671 \\
\hline 147 & 06916000 & MARAIS DES CYGNES R AT TRADING POST, KS & 0 & 141,000 & 1,600 & $4,848.20$ & 10,592 \\
\hline 148 & 06916500 & BIG SUGAR C AT FARLINVILLE, KS & 0 & 16,400 & 120 & 589.97 & 8,728 \\
\hline 149 & 06917000 & L OSAGE R AT FULTON, KS & 0 & 51,800 & 237 & 966.68 & 24,837 \\
\hline 150 & 06917060 & LITTLE OSAGE RIVER AT HORTON, MO & 0 & 43,700 & 364 & $1,197.50$ & 6,210 \\
\hline 151 & 06917240 & MARMATON R NR UNIONTOWN, KS & 0 & 8,710 & 68.4 & 279.91 & 5,844 \\
\hline 152 & 06917380 & MARMATON R NR MARMATON, KS & 0 & 67,900 & 280 & $1,281.80$ & 13,149 \\
\hline 153 & 06917500 & MARMATON R NR FORT SCOTT, KS & 0 & 30,200 & 293 & $1,151.10$ & 19,730 \\
\hline 154 & 06917560 & MARMATON RIVER NEAR RICHARDS, MO & 0.11 & 35,300 & 470 & 1,438 & 4,018 \\
\hline 155 & 06918060 & MARMATON RIVER NEAR NEVADA, MO & 0.03 & 33,800 & 944 & $2,232.40$ & 4,748 \\
\hline
\end{tabular}


Table 8. Basic statistics for daily mean streamflows of U.S. Geological Survey streamgages used in the regression analysis (map numbers 127-254), computed using SWToolbox (Kiang and others, 2018)._-Continued

[no., number; USGS, U.S. Geological Survey; MO, Missouri; R, River; NR, near; KS, Kansas; C, Creek; L, Little; CO, Colorado; HWY, Highway; NF, North Fork; AB, above; RE, Reservoir; SF, South Fork; OK, Oklahoma]

\begin{tabular}{|c|c|c|c|c|c|c|c|}
\hline $\begin{array}{c}\text { Map no. } \\
\text { (fig. 1) }\end{array}$ & USGS station number & USGS station name & Minimum & Maximum & Mean & $\begin{array}{l}\text { Standard } \\
\text { deviation }\end{array}$ & Count \\
\hline 156 & 06918070 & OSAGE RIVER ABOVE SCHELL CITY, MO & 0 & 153,000 & 5,240 & $9,698.70$ & 9,329 \\
\hline 157 & 06918460 & TURNBACK CREEK ABOVE GREENFIELD, MO & 4.48 & 23,700 & 259 & 554.58 & 18,628 \\
\hline 158 & 06919000 & SAC RIVER NEAR STOCKTON, MO & 0 & 79,800 & 986 & $2,308.40$ & 16,802 \\
\hline 159 & 06919500 & CEDAR CREEK NEAR PLEASANT VIEW, MO & 0 & 28,300 & 329 & $1,040.20$ & 25,931 \\
\hline 160 & 06921590 & SOUTH GRAND RIVER AT ARCHIE, MO & 0 & 25,500 & 298 & $1,133.80$ & 9,628 \\
\hline 161 & 06921600 & SOUTH GRAND RIVER AT URICH, MO & 0 & 27,200 & 448 & $1,473.20$ & 4,087 \\
\hline 162 & 06921720 & BIG CREEK NEAR BLAIRSTOWN, MO & 0 & 20,800 & 342 & $1,060.90$ & 8,533 \\
\hline 163 & 06921760 & SOUTH GRAND RIVER NEAR CLINTON, MO & 2.02 & 59,900 & 1,070 & $3,291.50$ & 7,075 \\
\hline 164 & 06922000 & SOUTH GRAND RIVER NEAR BROWNINGTON, MO & 0 & 60,300 & 1,050 & $2,806.30$ & 17,897 \\
\hline 165 & 07134100 & BIG SANDY CREEK NEAR LAMAR, CO & 0 & 1,460 & 13.3 & 27.249 & 13,213 \\
\hline 166 & 07139800 & MULBERRY C NR DODGE CITY, KS & 0 & 666 & 0.64 & 12.772 & 8,035 \\
\hline 167 & 07140700 & GUZZLERS GULCH NR NESS CITY, KS & 0 & 1,020 & 2.08 & 26.35 & 6,940 \\
\hline 168 & 07140850 & PAWNEE R NR BURDETT, KS & 0 & 3,830 & 9 & 87.74 & 12,784 \\
\hline 169 & 07141175 & BUCKNER C NR BURDETT, KS & 0 & 2,750 & 13.6 & 91.146 & 6,209 \\
\hline 170 & 07141200 & PAWNEE R AT ROZEL, KS & 0 & 14,300 & 54.6 & 354.09 & 33,575 \\
\hline 171 & 07141770 & WALNUT C NR ALEXANDER, KS & 0 & 1,550 & 14.5 & 62.052 & 6,575 \\
\hline 172 & 07141780 & WALNUT C AT NEKOMA, KS & 0 & 5,690 & 21.8 & 135.76 & 17,167 \\
\hline 173 & 07141900 & WALNUT C AT ALBERT, KS & 0 & 10,300 & 43.7 & 224.44 & 21,185 \\
\hline 174 & 07142300 & RATTLESNAKE C NR MACKSVILLE, KS & 0 & 7,330 & 20 & 85.207 & 20,819 \\
\hline 175 & 07142575 & RATTLESNAKE C NR ZENITH, KS & 0 & 13,600 & 39 & 176.91 & 15,706 \\
\hline 176 & 07143300 & COW C NR LYONS, KS & 0 & 16,800 & 76 & 354.84 & 25,157 \\
\hline 177 & 07143600 & L ARKANSAS R NR LITTLE RIVER, KS & 0 & 1,190 & 9.09 & 53.982 & 4,017 \\
\hline 178 & 07143665 & L ARKANSAS R AT ALTA MILLS, KS & 0 & 15,300 & 200 & 792.31 & 13,880 \\
\hline 179 & 07143672 & L ARKANSAS R AT HWY 50 NR HALSTEAD, KS & 0 & 10,800 & 201 & 745.53 & 7,670 \\
\hline 180 & 07144100 & L ARKANSAS R NR SEDGWICK, KS & 0.56 & 17,600 & 351 & $1,239.30$ & 8,401 \\
\hline 181 & 07144200 & L ARKANSAS R AT VALLEY CENTER, KS & 1.1 & 28,600 & 315 & $1,110.20$ & 34,334 \\
\hline 182 & 07144780 & NF NINNESCAH R AB CHENEY RE, KS & 0 & 39,700 & 139 & 516.96 & 18,628 \\
\hline 183 & 07144800 & NF NINNESCAH R NR CHENEY, KS & 0 & 10,300 & 165 & 451.11 & 4,749 \\
\hline 184 & 07145200 & SF NINNESCAH R NR MURDOCK, KS & 7.9 & 18,000 & 204 & 422.94 & 22,366 \\
\hline 185 & 07145500 & NINNESCAH R NR PECK, KS & 0.2 & 33,700 & 555 & $1,287.50$ & 9,131 \\
\hline
\end{tabular}


Table 8. Basic statistics for daily mean streamflows of U.S. Geological Survey streamgages used in the regression analysis (map numbers 127-254), computed using SWToolbox (Kiang and others, 2018).-Continued

[no., number; USGS, U.S. Geological Survey; MO, Missouri; R, River; NR, near; KS, Kansas; C, Creek; L, Little; CO, Colorado; HWY, Highway; NF, North Fork; AB, above; RE, Reservoir; SF, South Fork; OK, Oklahoma

\begin{tabular}{|c|c|c|c|c|c|c|c|}
\hline $\begin{array}{l}\text { Map no. } \\
\text { (fig. 1) }\end{array}$ & USGS station number & USGS station name & Minimum & Maximum & Mean & $\begin{array}{l}\text { Standard } \\
\text { deviation }\end{array}$ & Count \\
\hline 186 & 07145700 & SLATE C AT WELLINGTON, KS & 0 & 10,600 & 75.7 & 356.35 & 17,532 \\
\hline 187 & 07146570 & COLE C NR DEGRAFF, KS & 0 & 6,000 & 17.1 & 119.15 & 6,940 \\
\hline 188 & 07147070 & WHITEWATER R AT TOWANDA, KS & 0.3 & 49,400 & 206 & 975.14 & 18,628 \\
\hline 189 & 07147800 & WALNUT R AT WINFIELD, KS & 0 & 77,100 & 792 & $2,799.80$ & 21,550 \\
\hline 190 & 07148350 & $\begin{array}{l}\text { SALT FORK ARKANSAS RIVER NR WINCHESTER, } \\
\text { OK }\end{array}$ & 0 & 12,100 & 93.6 & 391.64 & 12,053 \\
\hline 191 & 07148400 & SALT FORK ARKANSAS RIVER NR ALVA, OK & 0 & 17,000 & 125 & 435.86 & 18,627 \\
\hline 192 & 07149000 & MEDICINE LODGE R NR KIOWA, KS & 0 & 9,660 & 146 & 338.56 & 25,994 \\
\hline 193 & 07151500 & CHIKASKIA R NR CORBIN, KS & 0 & 27,800 & 256 & 841.86 & 20,454 \\
\hline 194 & 07152000 & CHIKASKIA RIVER NEAR BLACKWELL, OK & 0 & 69,500 & 485 & $2,069.50$ & 14,975 \\
\hline 195 & 07153000 & BLACK BEAR CREEK AT PAWNEE, OK & 0 & 25,400 & 164 & 779.59 & 8,035 \\
\hline 196 & 07153100 & $\begin{array}{l}\text { RANCH CREEK AT CLEV DAM NR CLEVELAND, } \\
\text { OK }\end{array}$ & 0 & 4,840 & 10.4 & 88.99 & 6,574 \\
\hline 197 & 07155590 & CIMARRON R NR ELKHART, KS & 0 & 6,190 & 7.46 & 103.63 & 15,341 \\
\hline 198 & 07156010 & NF CIMARRON R AT RICHFIELD, KS & 0 & 6,100 & 5.25 & 103.79 & 5,479 \\
\hline 199 & 07157000 & CIMARRON RIVER NEAR MOCANE, OK & 0 & 17,900 & 97 & 316.42 & 8,036 \\
\hline 200 & 07157500 & CROOKED C NR ENGLEWOOD, KS & 0 & 12,700 & 25.4 & 167.41 & 26,977 \\
\hline 201 & 07157950 & CIMARRON RIVER NEAR BUFFALO, OK & 0 & 12,500 & 155 & 454.03 & 5,844 \\
\hline 202 & 07157960 & BUFFALO CREEK NEAR LOVEDALE, OK & 0 & 3,830 & 12.9 & 85.981 & 9,497 \\
\hline 203 & 07158000 & CIMARRON RIVER NEAR WAYNOKA, OK & 0 & 51,600 & 346 & $1,437.70$ & 14,610 \\
\hline 204 & 07159000 & TURKEY CREEK NEAR DRUMMOND, OK & 0 & 10,400 & 50.1 & 276.2 & 8,035 \\
\hline 205 & 07165700 & VERDIGRIS R NR MADISON, KS & 0 & 11,900 & 129 & 470.87 & 7,305 \\
\hline 206 & 07165750 & VERDIGRIS R NR VIRGIL, KS & 0 & 8,440 & 190 & 579.47 & 4,747 \\
\hline 207 & 07166500 & VERDIGRIS R NR ALTOONA, KS & 0 & 57,000 & 665 & $2,486.90$ & 7,305 \\
\hline 208 & 07167000 & FALL R NR EUREKA, KS & 0 & 31,300 & 194 & 810.09 & 10,593 \\
\hline 209 & 07167500 & OTTER C AT CLIMAX, KS & 0 & 21,700 & 85.6 & 488.59 & 25,568 \\
\hline 210 & 07168500 & FALL R AT FALL RIVER, KS & 0 & 54,000 & 464 & 2248.5 & 4,170 \\
\hline 211 & 07169500 & FALL R AT FREDONIA, KS & 0 & 39,300 & 536 & $1,944.40$ & 3,653 \\
\hline 212 & 07169800 & ELK R AT ELK FALLS, KS & 0 & 47,500 & 153 & 717.31 & 18,263 \\
\hline 213 & 07170000 & ELK R NR ELK CITY, KS & 0 & 56,200 & 307 & $1,513.50$ & 10,958 \\
\hline
\end{tabular}


Table 8. Basic statistics for daily mean streamflows of U.S. Geological Survey streamgages used in the regression analysis (map numbers 127-254), computed using SWToolbox (Kiang and others, 2018)._-Continued

[no., number; USGS, U.S. Geological Survey; MO, Missouri; R, River; NR, near; KS, Kansas; C, Creek; L, Little; CO, Colorado; HWY, Highway; NF, North Fork; AB, above; RE, Reservoir; SF, South Fork; OK, Oklahoma]

\begin{tabular}{|c|c|c|c|c|c|c|c|}
\hline $\begin{array}{l}\text { Map no. } \\
\text { (fig. 1) }\end{array}$ & USGS station number & USGS station name & Minimum & Maximum & Mean & $\begin{array}{l}\text { Standard } \\
\text { deviation }\end{array}$ & Count \\
\hline 214 & 07170500 & VERDIGRIS R AT INDEPENDENCE, KS & 0 & 106,000 & 1,570 & $4,823.30$ & 12,416 \\
\hline 215 & 07170700 & BIG HILL C NR CHERRYVALE, KS & 0 & 10,700 & 27.3 & 183.33 & 7,670 \\
\hline 216 & 07171000 & VERDIGRIS RIVER NEAR LENAPAH, OK & 0 & 134,000 & 2,720 & $7,465.10$ & 3,653 \\
\hline 217 & 07172000 & CANEY R NR ELGIN, KS & 0 & 79,200 & 282 & $1,180.40$ & 28,124 \\
\hline 218 & 07173000 & CANEY RIVER NEAR HULAH, OK & 0 & 31,900 & 398 & $1,578.80$ & 4,383 \\
\hline 219 & 07174000 & LITTLE CANEY RIVER NEAR COPAN, OK & 0 & 22,500 & 246 & $1,072.80$ & 5,113 \\
\hline 220 & 07174200 & $\begin{array}{l}\text { LITTLE CANEY RIVER BLW COTTON CR, NR CO- } \\
\text { PAN, OK }\end{array}$ & 0 & 22,000 & 278 & 943.3 & 7,671 \\
\hline 221 & 07174600 & SAND CREEK AT OKESA, OK & 0 & 13,200 & 82.6 & 407.7 & 12,053 \\
\hline 222 & 07176000 & VERDIGRIS RIVER NEAR CLAREMORE, OK & 0 & 181,000 & 4,020 & 10,398 & 4,748 \\
\hline 223 & 07176500 & BIRD CREEK AT AVANT, OK & 0 & 28,500 & 197 & 959.41 & 11,323 \\
\hline 224 & 07177000 & HOMINY CREEK NEAR SKIATOOK, OK & 0 & 30,000 & 179 & 842.23 & 13,500 \\
\hline 225 & 07177500 & BIRD CREEK NEAR SPERRY, OK & 0 & 62,800 & 488 & $2,039.80$ & 16,437 \\
\hline 226 & 07179500 & NEOSHO R AT COUNCIL GROVE, KS & 0 & 34,000 & 123 & 848.84 & 9,132 \\
\hline 227 & 07180000 & COTTONWOOD R NR MARION, KS & 0 & 30,600 & 115 & 654.39 & 10,593 \\
\hline 228 & 07180500 & CEDAR C NR CEDAR POINT, KS & 0 & 10,900 & 59.9 & 284.08 & 28,490 \\
\hline 229 & 07181500 & M CREEK NR ELMDALE, KS & 0 & 5,510 & 42.2 & 234.35 & 4,018 \\
\hline 230 & 07182400 & NEOSHO R AT STRAWN, KS & 0 & 274,000 & 1,360 & $5,702.70$ & 5,113 \\
\hline 231 & 07183000 & NEOSHO R NR IOLA, KS & 0 & 344,000 & 1,600 & $5,292.50$ & 18,443 \\
\hline 232 & 07183100 & OWL C NR PIQUA, KS & 0 & 15,600 & 112 & 592.74 & 3,652 \\
\hline 233 & 07183500 & NEOSHO R NR PARSONS, KS & 0 & 366,000 & 2,490 & $6,930.10$ & 14,610 \\
\hline 234 & 07184000 & LIGHTNING C NR MCCUNE, KS & 0 & 42,400 & 171 & 804.63 & 23,741 \\
\hline 235 & 07184500 & LABETTE C NR OSWEGO, KS & 0 & 13,400 & 196 & 790.98 & 4,018 \\
\hline 236 & 07185000 & NEOSHO RIVER NEAR COMMERCE, OK & 0 & 251,000 & 3,730 & $8,862.50$ & 8,035 \\
\hline 237 & 07185500 & STAHL CREEK NEAR MILLER, MO & 0 & 422 & 2.87 & 14.153 & 9,132 \\
\hline 238 & 07185700 & SPRING RIVER AT LA RUSSELL, MO & 17 & 19,900 & 268 & 552.81 & 12,298 \\
\hline 239 & 07185765 & SPRING RIVER AT CARTHAGE, MO & 12.1 & 28,700 & 409 & 918.95 & 10,743 \\
\hline 240 & 07186000 & SPRING RIVER NEAR WACO, MO & 4.5 & 108,000 & 958 & $2,684.40$ & 33,603 \\
\hline 241 & 07186400 & CENTER CREEK NEAR CARTERVILLE, MO & 9.7 & 10,000 & 209 & 449.2 & 10,227 \\
\hline 242 & 07187000 & SHOAL CREEK ABOVE JOPLIN, MO & 15 & 36,700 & 426 & 826.9 & 27,394 \\
\hline
\end{tabular}


Table 8. Basic statistics for daily mean streamflows of U.S. Geological Survey streamgages used in the regression analysis (map numbers 127-254), computed using SWToolbox (Kiang and others, 2018).-Continued

[no., number; USGS, U.S. Geological Survey; MO, Missouri; R, River; NR, near; KS, Kansas; C, Creek; L, Little; CO, Colorado; HWY, Highway; NF, North Fork; AB, above; RE, Reservoir; SF, South Fork; OK, Oklahoma]

\begin{tabular}{|c|c|c|c|c|c|c|c|}
\hline $\begin{array}{c}\text { Map no. } \\
\text { (fig. 1) }\end{array}$ & USGS station number & USGS station name & Minimum & Maximum & Mean & $\begin{array}{l}\text { Standard } \\
\text { deviation }\end{array}$ & Count \\
\hline 243 & 07187500 & SHOAL CREEK NEAR JOPLIN, MO & 16 & 12,000 & 398 & 673.78 & 6,209 \\
\hline 244 & 07188000 & SPRING RIVER NEAR QUAPAW, OK & 5.8 & 169,000 & 2,010 & $4,923.90$ & 16,436 \\
\hline 245 & 07189500 & NEOSHO RIVER NEAR GROVE, OK & 34 & 130,000 & 6,250 & 12,135 & 5,113 \\
\hline 246 & 07191000 & BIG CABIN CREEK NEAR BIG CABIN, OK & 0.1 & 46,300 & 325 & $1,465.60$ & 13,879 \\
\hline 247 & 07191222 & BEATY CREEK NEAR JAY, OK & 0 & 6,890 & 51.4 & 212.34 & 6,575 \\
\hline 248 & 07192000 & PRYOR CREEK NEAR PRYOR, OK & 0 & 26,600 & 138 & 695.89 & 5,478 \\
\hline 249 & 07232500 & BEAVER RIVER NEAR GUYMON, OK & 0 & 14,700 & 25.5 & 249.24 & 14,245 \\
\hline 250 & 07233000 & COLDWATER CREEK NEAR HARDESTY, OK & 0 & 11,300 & 16.2 & 203.42 & 8,766 \\
\hline 251 & 07234000 & BEAVER RIVER AT BEAVER, OK & 0 & 39,900 & 103 & 708.73 & 14,975 \\
\hline 252 & 07234100 & CLEAR CREEK NEAR ELMWOOD, OK & 0 & 5,480 & 7.47 & 98.423 & 5,114 \\
\hline 253 & 07234500 & BEAVER RIVER NEAR FORT SUPPLY, OK & 0 & 24,200 & 193 & 844.92 & 4,748 \\
\hline 254 & 07236000 & WOLF CREEK NEAR FARGO, OK & 0 & 21,800 & 65.8 & 399.39 & 12,054 \\
\hline
\end{tabular}




\section{Flow-Duration Statistics}

The flow-duration curve is a cumulative frequency curve that indicates the percentage of time a specified streamflow was equaled or exceeded during a given period (Searcy, 1959). Flow durations are first calculated by sorting individual flows for a period of record from the largest to the smallest value. Next, the frequencies of exceedance are then calculated using the Weibull formula for computing plotting position (Helsel and others, 2020):

$$
P=100 \cdot\left[\frac{M}{n+1}\right],
$$

where

$$
\begin{aligned}
& P \quad \text { is the probability that a given flow will be } \\
& \text { equaled or exceeded (percentage), } \\
& M \quad \text { is the ranked position (dimensionless), and } \\
& n \quad \text { is the number of events for the period of } \\
& \text { record (dimensionless). }
\end{aligned}
$$

Flow durations summarize past hydrologic events and rank them in order; however, if the streamflow during the period for which the flow-duration curve is based is a suitably long period (at least 10 years, but 30 years or more is desirable), the statistics can be used as an indicator of likely future conditions (Searcy, 1959). Flow durations for this report were calculated using daily mean values of streamflow for complete water years through September 2017. Flow durations in this report were computed for the 0.01-, 0.1-, 2-, 5-, 10-, 25-, 50-, 75-, 90-, 95-, 99-, 99.9-, and 99.99-percent exceedance intervals. An example of a flow-duration curve is shown in figure 7 , and in appendix 1, tables 1.1 and 1.2 provide the tabular calculations for all the streamgages used in this report.

\section{Low-Flow Frequency Statistics}

To estimate low-flow streamflows for selected recurrence intervals at streamgages in Kansas (for example, the 7Q10), a low-flow frequency analysis was done. For this report, low-flow frequencies were estimated for annual 1-, 7-, and 30-day flows at recurrence intervals of 5, 10, 20, and 50 years. Monthly (January, February, and so on) 1-, 7-, and 30-day flows at recurrence intervals of 5, 10, 20, and 50 years also were estimated. The magnitude and frequency of low flows are computed for a streamgage by relating a specific number of consecutive daily mean streamflows during a selected period (annually or monthly in this report) to a minimum nonexceedance probability or recurrence interval (Eash and Barnes, 2017).

\section{$n$-Day Analysis}

The low-flow frequency statistics in this report are based on the $n$-day, $Y$-year frequency statistic of daily mean streamflow for a climatic year. The climatic year (April 1-March 31) was used as the annual period for computing low-flow statistics. The climatic year is used for low-flow frequency investigation because low-flow events typically happen during the late summer through the winter months in Kansas. Use of the climatic year helps avoid the possibility of using one low-flow event that occurs during the fall months (September, October, and November) and has the potential to cross water years (October 1-September 30).

The mean streamflow for any $n$-day period throughout the climatic year is computed, and the minimum value is used for that period. The recurrence interval (expressed as $Y$-years) indicates the mean number of years between occurrences of a selected low-flow value; for example, the 7Q10 low-flow statistic is the annual minimum mean streamflow for 7 consecutive days that is expected to not be exceeded once during any 10-year period (Southard, 2013). This low-flow statistic naming convention does not indicate that a particular low-flow magnitude occurs on a regular yearly interval at a given streamgage. It can be more accurately described as a probability. The probability is the reciprocal of the annual nonexceedance interval, expressed as $1 / Y$ (Southard, 2013); therefore, a 7Q10 is the 7-day annual minimum mean flow that has a 10-percent chance of occurring in any given year and for which the mean time between occurrences is 10 years.

An example of the 7Q10 statistic that shows the annual minimum 7-day mean flows for the USGS streamgage Little Blue River near Barnes, Kansas (06884400), between climatic years 1960 and 2017 is provided in figure 8. Also shown in figure 8 is the $7 \mathrm{Q} 10$ that was computed from those data. From figure 8 , seven of the annual minimum 7-day mean flows fell to less than the calculated 7Q10. These seven occurrences are not evenly distributed across the time series. The first occurrence happens in 1965, but the second does not occur until 1989. The third occurrence happens shortly after the second (in 1992), but the fourth does not happen until 2007. The final three occurrences then happen consecutively between 2013 and 2015. As shown in figure 8, these statistics (the 7Q10 in this example) do not occur at a regular interval, but they have a probability of occurring each year.

For this report, low-flow frequencies were calculated for annual and monthly $n$-day durations of 1-, 7-, and 30-day intervals. Low-flow recurrence intervals were computed at 5 , 10,20 , and 50 years, with the corresponding probabilities being $20,10,5$, and 2 percent. A frequency examination was done on each $n$-day annual series using a log-Pearson Type III distribution, as indicated by Riggs (1972) and Barnes (1979). The computer program SWToolbox was used to compute the lowflow frequency statistics and fit them to a log-Pearson Type III distribution in this study. More detailed information on the log-Pearson Type III distribution is provided in "Guidelines for Determining Flood Flow Frequency-Bulletin 17C" (England 


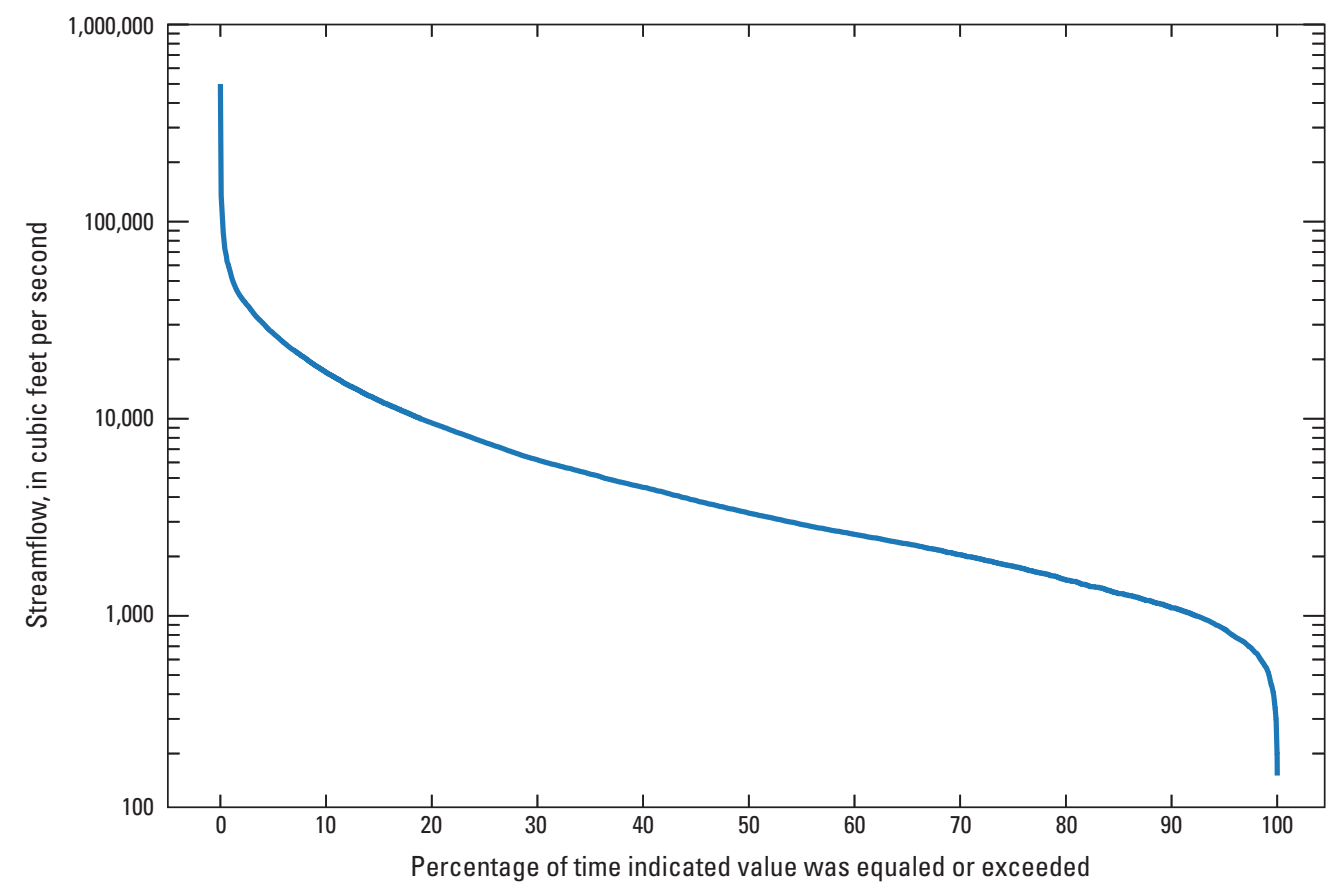

Figure 7. Flow-duration curve for the U.S. Geological Survey streamgage Kansas River at De Soto, Kansas (06892350).

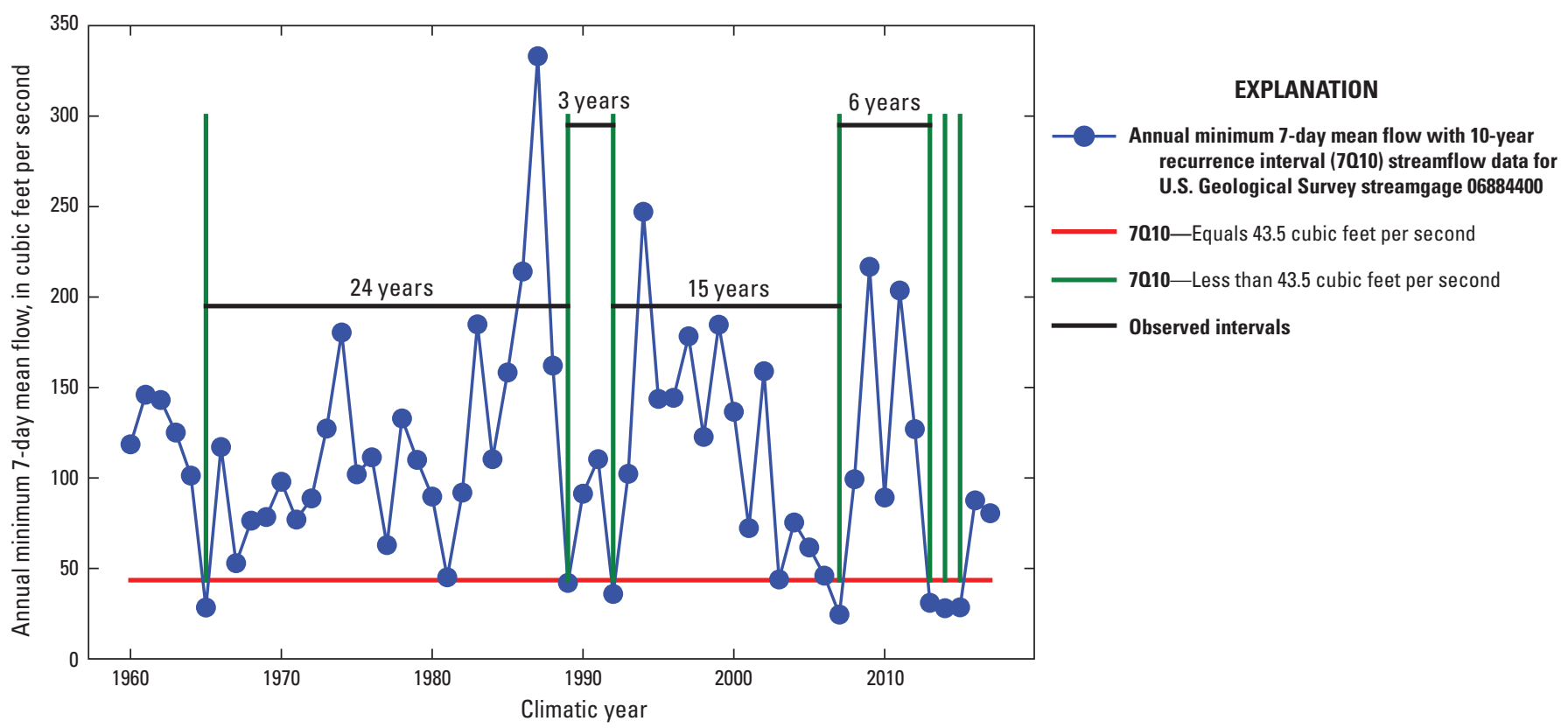

Figure 8. Annual minimum 7-day mean streamflow and calculated 7010 at U.S. Geological Survey streamgage Little Blue River near Barnes, Kansas (06884400). 
and others, 2019). Data that were output by the software program SWToolbox were reviewed for quality assurance. In appendix 2, table 2.1 provides the low-flow frequency statistics for streamgages that were used in the regression analysis, table 2.2 provides the monthly low-flow frequency statistics for streamgages that were used in the regression analysis, and table 2.3 provides the low-flow frequency statistics for all the active streamgages in the State of Kansas.

\section{Trend Analysis}

The SWToolbox software program uses the MannKendall test to test the time-series data for trends (for more information on this test, see Kendall [1938] and Helsel and others [2020]). This feature of SWToolbox was used for the time-series data in this report. The Mann-Kendall test was used to determine statistical significance of monotonic trends in annual 1-day, 7-day, and 30-day mean low flows with time. A trend was considered statistically significant for a probability value ( $p$-value) of less than or equal to 0.05 . SWToolbox also computes the Kendall's tau statistic to determine correlation. If the datasets indicate perfect positive correlation, then $\operatorname{tau}=1$; if there is perfect negative correlation, then tau $=-1$; and if there is no correlation, then tau $=0$ (Gotvald, 2016).

An important limitation of the Mann-Kendall statistical test is when it is used on streamflow data that have a considerable number of days of zero flows. The tau and its corresponding $p$-value become undependable, and interpretation of these numbers becomes weak because of ties in the data (Barbie and others, 2012; Gotvald, 2016). The trend analysis of minimum 1-day mean flows for 17 streamgages, minimum 7-day mean flows for 14 streamgages, and minimum 30-day mean flows for 7 streamgages produced tau and $p$-values that may be difficult to interpret because of the presence of several zero flows. In appendix 2, table 2.4 lists the results of the Mann-Kendall statistical test for trends on the streamgages used in the regression analysis.

When reviewing hydrologic records over short-term periods of a few years to a few decades, trends commonly appear (Lins and others, 2010); however, when these records are reviewed in the context of decades to centuries, shortterm trends may be recognized as being part of much longer term fluctuations (Lins and others, 2010). The Mann-Kendall test is more suitable for long-term hydrologic time-series records than short-term records. This test can be applied to shorter hydrologic time-series records; however, tests applied to shorter records may not deliver information that is useful. Mann-Kendall tests that are applied to shorter hydrologic time-series records may not identify a statistically significant trend even if a large increase or decrease in flow has occurred (Gotvald, 2016). Any trend identified by a test applied to a shorter time-series record may not indicate any practical importance (Gotvald, 2016); therefore, long-term hydrologic time-series records are better for trend assessments. Streamgages with 30 or more years of streamflow records are considered long-term streamgages by the USGS
(U.S. Geological Survey, 2020); therefore, this study designates long-term streamgages as having at least 30 or more years of streamflow records.

The USGS streamgage Little Blue River near Barnes, Kans. (06884400), is a long-term streamgage that has a record length of 58 years. Although a linear regression curve, which was included to offer a visual representation of possible trends, shows a small downward slope for the annual minimum 7-day mean flow for the period of record from climatic year 1960 to 2017, the Mann-Kendall test indicated no statistically significant trend (table 2.4; fig. 9A). The criteria used to determine the significance of the trend was the $p$-value produced by the Mann-Kendall test. A $p$-value of 0.05 or below indicates a significant trend, whereas a $p$-value above 0.05 indicates a nonsignificant trend. To demonstrate how period of record and hydrologic conditions measured in that record can affect the trend assessment, a trend analysis for various subsets of the record was completed. For the 20-year period between 1968 and 1987, the analysis indicated an upward trend $(\mathrm{tau}=0.4211$, $p$-value $=0.0104$; fig. 9B). For the 22-year period between 1994 and 2015, the analysis indicated a downward trend (tau $=-0.3853, p$-value $=0.0131$; fig. 9C). A trend analysis for the 30-year period from 1988 to 2017 indicated no significant trend (tau $=-0.2, p$-value $=0.1250$; fig. $D$ ). These results highlight the importance of long-term data-collection programs to accurately measure hydrologic trends.

Of the 254 streamgages used in the regression analysis, 77 indicated a significant trend for the 7-day annual minimum flow. An upward trend was seen at 56 streamgages, whereas a downward trend was observed at 21 streamgages. Of the 77 streamgages that indicated a significant trend, 47 had 30 years or more of streamflow record, whereas 30 had between 10 and 29 years of streamflow record. It is possible the trends observed at these streamgages are affected by changing climatological conditions within the study area. To evaluate the changes in climate in the study area, a Mann-Kendall test was completed on the annual mean air temperature and the annual measured precipitation for the State of Kansas and the National Oceanic and Atmospheric Administration, National Centers for Environmental Information climate divisions that are within or near Kansas.

The datasets for the annual mean air temperature and the annual measured precipitation contained data from 1895 to 2017 . The data for air temperature and precipitation were obtained from the National Oceanic and Atmospheric Administration, National Centers for Environmental Information website (National Oceanic and Atmospheric Administration, National Centers for Environmental Information, 2020). There are 20 total climate divisions within the study area (fig. 10). The trend analysis for annual mean air temperature indicated that 17 of the 20 climate divisions indicate a significant upward trend (fig. 10). The Southeast (Kansas), West Ozarks (Missouri), and Northeast (Oklahoma) climate divisions indicated no significant trend in the observed air temperature data. Conversely, the trend analysis for the annual measured precipitation indicated that 

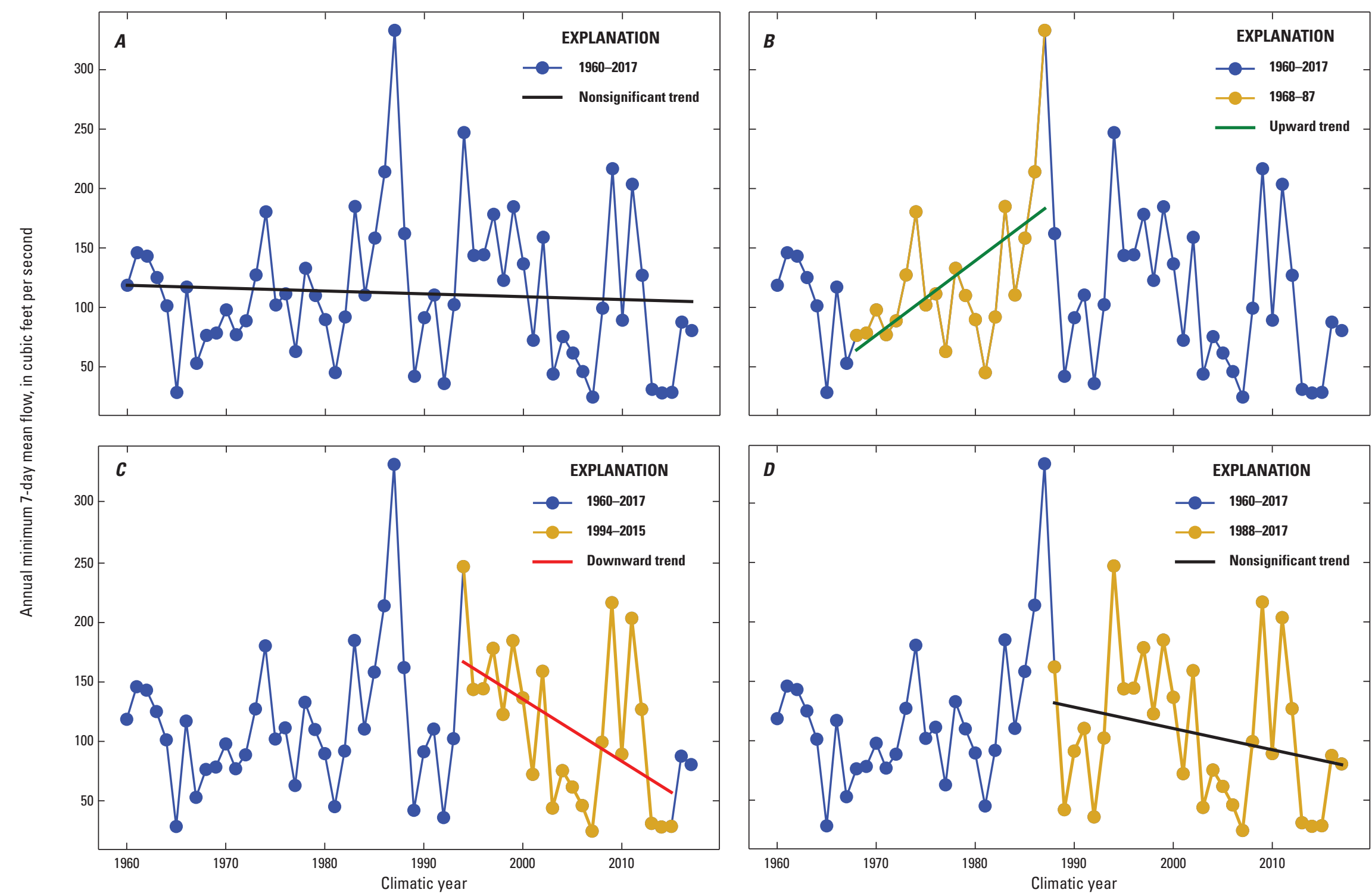

Figure 9. Selected periods of record for U.S. Geological Survey streamgage Little Blue River near Barnes, Kansas (06884400), for which a Mann-Kendall test for detection of trends was assessed. $A, 1960-2017 ; B, 1968-87 ; C, 1994-2015 ; D, 1988-2017$. 


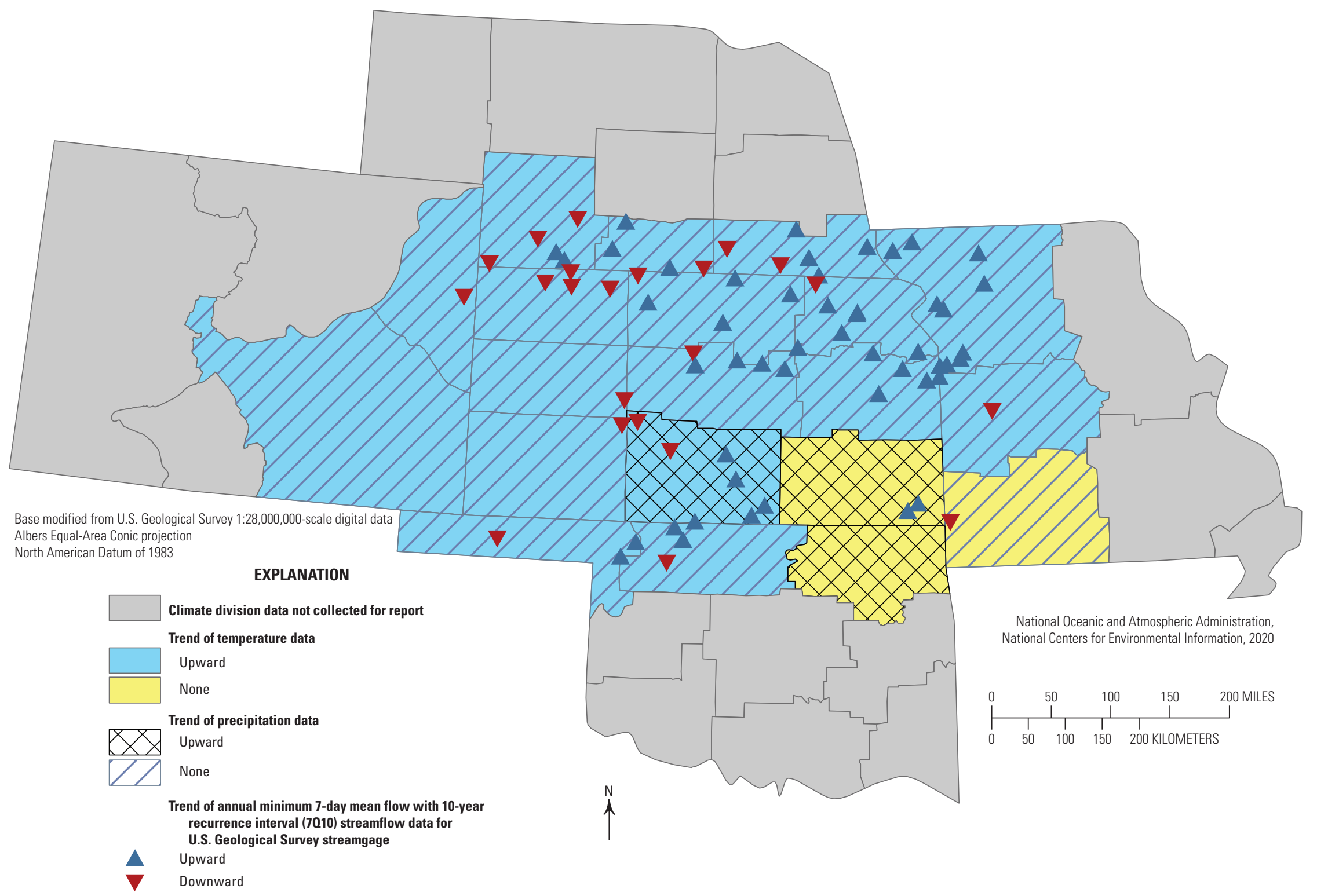

Figure 10. Annual mean temperature and annual mean precipitation trends by National Oceanic and Atmospheric Administration climate divisions within the study area, 1987-2017, and annual minimum 7-day mean flow with 10-year recurrence interval streamflow trends at streamgages for their period of record through 2017. 
17 of the 20 climate divisions indicated no significant trend (fig. 10). The South Central (Kansas), Southeast (Kansas), and Northeast (Oklahoma) climate divisions indicated a significant upward trend for the precipitation data.

The upward trends in the climate data could explain the upward trends seen in the streamgages that indicate a similar trend; however, the trends in the climate data do not explain the downward trend observed at other streamgages. One potential source of effect on the streamgages with a downward trend is increased groundwater pumping. Juracek and others (2017) stated that a likely or possible diminished condition for streamflow in the Cimarron River and Rattlesnake Creek Basins, located in the Southwest (Kansas) and Northwest (Oklahoma) climate divisions, was caused by groundwater declines that result from groundwater pumping for irrigated agriculture. Shown in figure 4 are the streamgages in this report that indicate a significant trend for the annual minimum 7-day mean flow and the land uses near those streamgages.

An analysis of the causes of the trends in annual 1-day, 7-day, and 30-day low flows in Kansas is outside the scope of this report. Two recent extensive studies on the factors and trends affecting streamflows in Kansas have been published. Juracek (2015) and Juracek and Eng (2017) concluded that an annual precipitation explanation for changing streamflows in Kansas was not supported because of the lack of a trend in the precipitation data. Both studies also determined that groundwater pumping was the likely reason for lower streamflow conditions occurring in western and central Kansas. Juracek and Eng (2017) also noted that most streamgages that were determined to have a lower streamflow condition are in the western half of Kansas. Because of the presence of numerous zero flows in the dataset and several periods of record being less than 30 years in length, no streamflow data were excluded from this study because of the presence of a significant trend in streamflow.

\section{Regression Equation Development}

In a regional regression study, dividing a large study area into subregions that are fairly homogenous in terms of low-flow hydrology can help to reduce error in the regression equations (Eash and Barnes, 2017). Because two flow regions were developed in the Painter and others (2017) study, the same two flow regions were used in this study. Because of the presence of zero flows in the dataset, a multiple linear regression analysis could not be used because of the errors caused by log-transforming zero. Other regression techniques were explored, and the left-censored and logistic regression techniques were chosen as the most appropriate methods to deal with the substantial amount of zero flows in the dataset.

\section{Multiple Linear Regression}

Multiple linear regression analysis has been used by the USGS and other researchers throughout the United States and elsewhere to develop equations for estimating streamflow statistics at ungaged locations (Perry and others, 2004a). In a regression analysis, a streamflow statistic, also known as the dependent variable, for a collection of streamgages is correlated statistically to the basin characteristics, also known as the independent variables (Perry and others, 2004a). The result is an equation that can be used to estimate the streamflow statistic for locations where no streamflow data are present. Regression equations can be developed by using several types of multiple linear regression analyses. Choosing one analysis over another depends on the characteristics of the data to be used in the analysis and the assumptions made in that analysis. The multiple linear regression equation is represented by the following general formula:

$$
Y_{i}=b_{0}+b_{1} X_{1}+b_{2} X_{2}+\ldots+b_{n} X_{n}+e_{i}
$$

where

$$
\begin{aligned}
Y_{i} & \text { is the dependent variable for location } i, \\
b_{0} \text { to } b_{n} & \text { are the } n+1 \text { regression-model coefficients, } \\
X_{1} \text { to } X_{n} & \text { are the } n \text { independent variables, and } \\
e_{i} & \begin{array}{c}
\text { is the error for location } i \text { (Perry and } \\
\text { others, 2004a). }
\end{array}
\end{aligned}
$$

Assumptions for the use of multiple linear regression analyses are as follows:

1. the model adequately describes the linear relation between the dependent and independent variables,

2. the mean of $e_{i}$ is zero,

3. the variance of $e_{i}$ is constant and independent of the values of $X_{n}$,

4. the values of $e_{i}$ are normally distributed, and

5. the values of $e_{i}$ are independent of each other (Iman and Conover, 1983; Eash and Barnes, 2017).

Streamflow and basin characteristics are usually lognormally distributed when used in hydrologic regression analysis (Perry and others, 2004a). Logarithmic transformations of variables is usually required to satisfy regression assumption 4 .

\section{Left-Censored Regression}

Left-censored regression, which also can be referred to as a Tobit analysis or Tobit regression, can be used to include zero values in an analysis that includes logarithmic transformation (Perry and others, 2004a). Censored data are data values that are less than a threshold value (Perry and other, 2004a) These censored values are then increased to the 
threshold value so that the data can be logarithmically transformed (Perry and others, 2004a). Left-censored regression is comparable to multiple linear regression, except the regression coefficients are fit via maximum-likelihood estimation (MLE; Eash and Barnes, 2017; Helsel and others, 2020). MLE is similar to a curve-matching procedure, where a probability distribution is best fitted to the observed data (Eash and Barnes, 2017). "MLE assumes that residuals are normally distributed around the regression line for the estimation of the slope and intercept, and the range of predicted values has constant variance" (Eash and Barnes, 2017, p. 26). More information on MLE is provided in Helsel and others (2020) and in Runkel and others (2004).

\section{Logistic Regression}

The response variable in a logistic regression is categorical or binary. In this study, a response value of zero was given to streamgages with no flow, and a response value of one was given to streamgages with flow. The probability of the response variable falling into one category or the other is examined to determine if it varies as a function of continuous independent variables (Eash and Barnes, 2017; Helsel and others, 2020). Computations from logistic regression will be between zero and one and are known as the probability $(p)$ of observing a response of one, or predicting flow at an ungaged location to be greater than zero (Eash and Barnes, 2017); thus, $(1-p)$ is the probability of observing a response of zero or predicting zero flow at an ungaged location (Eash and Barnes, 2017). The form of the logistic regression equation to predict zero flow is as follows:

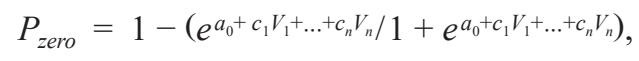

where

$$
\begin{aligned}
& P_{z e r o} \quad \text { is the probability of the low-flow frequency } \\
& \text { statistic being zero; } \\
& a_{0} \quad \text { is the regression-model constant; } \\
& e \quad \text { is the base of the natural logarithm value, } \\
& \text { approximately } 2.7183 \text {; } \\
& c_{1} \text { to } c_{n} \text { are the regression-model coefficients; and } \\
& V_{1} \text { to } V_{n} \quad \text { are the independent variables, or basin } \\
& \text { characteristics (Eash and Barnes, 2017). }
\end{aligned}
$$

Current applications of logistic regression that pertain to lowflow studies are provided in Funkhouser and others (2008), Martin and Arihood (2010), and Eash and Barnes (2017).

\section{Final Regression Equations}

Logistic and left-censored regression techniques were chosen for this study because several of the flow-duration and low-flow statistic values were calculated or estimated to be zero. The left-censored regression equations in this study were weighted based on the number of years of record available (Lorenz, 2014; Gotvald, 2017). Censored and uncensored values were used in the left-censored regression analyses. When datasets do not have censored values, weighted left-censored regression delivers the same results as weighted least squares regression (Gotvald, 2017; Helsel and others, 2020). Because of the ability of a streamgage to report continuous data lower than 0.1 cubic foot per second $\left(\mathrm{ft}^{3} / \mathrm{s}\right)$, the censoring threshold used to develop the left-censored regression equations was set at $0.1 \mathrm{ft} 3 / \mathrm{s}$ (Gotvald, 2017). Seven basin characteristics were used as independent variables in the final regression equations (table 6). These include contributing drainage area (CONTDA), BFI, crop land cover, mean soil permeability or saturated hydraulic conductivity of soil, reservoir storage, mean annual precipitation for 1895-2017 (PRECIPfull), and drainage basin slope. GIS software was required to measure most of the basin characteristics used as independent variables in the final regression equations in this report. The basin characteristics that were not measured with GIS software were BFI (Barlow and others, 2015) and PRECIPfull (PRISM Climate Group, 2020).

Final regression equations for the east and west regions of Kansas are listed in tables 9 and 10, along with the number of streamgages used in the regression analysis and select performance metrics. Explanations of the variables and their corresponding units of measure are listed in table 6. Most of the independent variables included in the final regression equations were statistically significant at the 95-percent confidence level and were not correlated with other independent variables used in the same equation. The only variable that was allowed outside of the 95-percent confidence interval was the CONTDA. This was done because including the CONTDA lowered the standard error (SE) of the equations when it was included. Because left-censored and logistic regression techniques were used to develop final equations, performance metrics are reported differently for each type of regression.

For the 17 logistic regression equations in the east region and the 43 logistic regression equations in the west region of Kansas, classification is reported, in percent, and the number of streamgages used to develop the equation also is listed. Classification is the predictive accuracy of streamgages correctly estimated to have zero flow. For the 53 left-censored regression equations in the east region and the 53 left-censored regression equations in the west region of Kansas, the SE, the SE range, and the pseudocoefficient of determination are reported, in percent. The number of streamgages censored in each analysis and the number of streamgages used to develop the equation also are listed.

The logistic regression equations in the east and west regions of Kansas (tables 9 and 10) should be used first to determine the probability of a specific low-flow statistic or flow-duration exceedance interval equaling zero for an ungaged location before the left-censored regression equation is used to estimate a specific low-flow statistic or flowduration exceedance interval. If the resulting probability is greater than or equal to 0.5 , then the value for that low-flow 
[classification, percentage of time equation correctly predicts zero flow; SE, standard error; \%, percent; RMSE, root mean square error; pseudo- $R^{2}$, pseudocoefficient of determination; 1Q10, 1-day mean flow with 10-year recurrence interval; $\mathrm{Q}$, flow; Ann, annual; $\mathrm{P}_{\text {zero }}$, probability of zero flow; $e$, base of natural logarithm, approximately equal to 2.7183 ; ln, natural logarithm; CONTDA, contributing drainage area; $B F I$, base-flow index; $L C 06 C R O P$, percentage of crop land cover; NA, not applicable; 7Q10, 7-day mean flow with 10-year recurrence interval; 30Q10, 30-day mean flow with 10-year recurrence interval; KSAT, saturated hydraulic conductivity; STORAGE, percentage of reservoir storage; FD, flow duration; 99.99, 99.99\% interval; 99.90, 99.90\% interval; 99, 99\% interval; 95, 95\% interval; 90, 90\% interval; $75,75 \%$ interval; $50,50 \%$ interval; $25,25 \%$ interval; PRECIPfull, mean annual precipitation for $1895-2017 ; 10,10 \%$ interval; $5,5 \%$ interval; $2,2 \%$ interval; $0.1,0.1 \%$ interval; $0.01,0.01 \%$ interval]

\begin{tabular}{|c|c|c|c|c|c|c|c|c|}
\hline \multirow{2}{*}{$\begin{array}{l}\text { East (region 1) } \\
\text { flow statistic }\end{array}$} & \multirow[b]{2}{*}{ Regression model } & \multirow[b]{2}{*}{ Regression type } & \multicolumn{6}{|c|}{ Performance metrics } \\
\hline & & & Classification & SE (\%) & RMSE & Pseudo- $R^{2}$ & $\begin{array}{l}\text { Number } \\
\text { censored }\end{array}$ & $\begin{array}{c}\text { Number of } \\
\text { streamgages }\end{array}$ \\
\hline 1Q10_Ann_P zero ${ }^{*}$ & $\begin{aligned} 1- & \left(e^{1.27+0.04 \ln (C O N T D A)+2.59 \ln (B F I)}\right. \\
& +0.75 \ln (L C 06 C R O P) / 1+e^{1.27+0.04 \ln } \\
& (C O N T D A)+2.59 \ln (B F I)+0.75 \ln (L C 06 C R O P))\end{aligned}$ & Logistic & 75.3 & $\mathrm{NA}$ & NA & NA & NA & 181 \\
\hline 7Q10_Ann_P zero ${ }^{*}$ & $\begin{aligned} 1- & \left(e^{0.97+0.06 \ln (C O N T D A)+2.42 \ln (B F I)}\right. \\
& +0.82 \ln (L C 06 C R O P) / 1+e^{0.97+0.06 \ln } \\
& (C O N T D A)+2.42 \ln (B F I)+0.82 \ln (L C 06 C R O P))\end{aligned}$ & Logistic & 79.2 & NA & NA & NA & NA & 181 \\
\hline 30Q10_Ann_P $\mathrm{P}_{\text {zero }} *$ & $\begin{aligned} 1- & \left(e^{1.30+0.23 \ln (C O N T D A)+2.10 \ln (B F I)}\right. \\
& +0.51 \ln (L C 06 C R O P) / 1+e^{1.30+0.23 \ln } \\
& (C O N T D A)+2.10 \ln (B F I)+0.5 \ln (L C 06 C R O P))\end{aligned}$ & Logistic & 87.6 & NA & NA & NA & NA & 181 \\
\hline 1Q10_Ann & $\begin{array}{l}0.42+0.96 \ln (C O N T D A) \\
-0.81 \ln (K S A T)+5.06 \ln (B F I)\end{array}$ & Left censored & NA & 1,485 & 2.32 & 0.7229 & 100 & 181 \\
\hline 7Q10_Ann & $\begin{array}{l}-0.16+1.01 \ln (C O N T D A) \\
\quad-0.73 \ln (K S A T)+4.55 \ln (B F I)\end{array}$ & Left censored & NA & 1,133 & 2.20 & 0.7229 & 94 & 181 \\
\hline 30Q10_Ann & $\begin{array}{l}0.20+0.81 \ln (C O N T D A) \\
\quad-0.77(K S A T)+4.10 \ln (B F I) \\
\quad+0.40 \ln (L C 06 C R O P)\end{array}$ & Left censored & NA & 556.9 & 1.86 & 0.7518 & 75 & 181 \\
\hline 1Q10_January & $\begin{array}{l}-0.62+0.79 \ln (C O N T D A) \\
\quad+2.37 \ln (B F I)\end{array}$ & Left censored & NA & 200.6 & 1.27 & 0.7753 & 34 & 181 \\
\hline 7Q10_January & $\begin{array}{l}-0.73+0.82 \ln (C O N T D A) \\
\quad+2.28 \ln (B F I)\end{array}$ & Left censored & NA & 193.4 & 1.25 & 0.7814 & 30 & 181 \\
\hline 30Q10_January & $\begin{array}{l}-0.54+0.81 \ln (C O N T D A) \\
\quad+1.99(B F I)\end{array}$ & Left censored & NA & 175.5 & 1.19 & 0.7737 & 20 & 181 \\
\hline 1Q10_February & $\begin{array}{l}-0.75+0.79 \ln (C O N T D A) \\
\quad+2.04 \ln (B F I)\end{array}$ & Left censored & NA & 204.4 & 1.28 & 0.7462 & 29 & 181 \\
\hline 7Q10_February & $\begin{array}{l}-0.68+0.81 \ln (C O N T D A) \\
\quad+1.98 \ln (B F I)\end{array}$ & Left censored & NA & 193.3 & 1.25 & 0.7547 & 25 & 181 \\
\hline 30Q10_February & $\begin{array}{l}-0.29+0.77 \ln (C O N T D A) \\
\quad+1.54 \ln (B F I)\end{array}$ & Left censored & NA & 161.3 & 1.13 & 0.7413 & 9 & 181 \\
\hline 1Q10_March & $\begin{array}{l}-0.65+0.82 \ln (C O N T D A) \\
\quad+1.89 \ln (B F I)\end{array}$ & Left censored & NA & 194.9 & 1.25 & 0.7477 & 22 & 181 \\
\hline
\end{tabular}


Table 9. Regression equations for estimating selected low-flow frequency statistics for unregulated streams in the east region of Kansas.-Continued

[classification, percentage of time equation correctly predicts zero flow; SE, standard error; \%, percent; RMSE, root mean square error; pseudo- $R^{2}$, pseudocoefficient of determination; $1 \mathrm{Q} 10,1$-day mean flow with 10-year recurrence interval; $\mathrm{Q}$, flow; Ann, annual; $\mathrm{P}_{\text {zero }}$, probability of zero flow; $e$, base of natural logarithm, approximately equal to $2.7183 ; \ln$, natural logarithm; CONTDA, contributing drainage area; $B F I$, base-flow index; $L C 06 C R O P$, percentage of crop land cover; NA, not applicable; 7Q10, 7-day mean flow with 10-year recurrence interval; 30Q10, 30-day mean flow with 10-year recurrence interval; KSAT, saturated hydraulic conductivity; STORAGE, percentage of reservoir storage; FD, flow duration; $99.99,99.99 \%$ interval; $99.90,99.90 \%$ interval; $99,99 \%$ interval; $95,95 \%$ interval; $90,90 \%$ interval; $75,75 \%$ interval; 50, 50\% interval; 25, 25\% interval; PRECIPfull, mean annual precipitation for $1895-2017 ; 10,10 \%$ interval; 5, 5\% interval; $2,2 \%$ interval; $0.1,0.1 \%$ interval; $0.01,0.01 \%$ interval $]$

\begin{tabular}{|c|c|c|c|c|c|c|c|c|}
\hline \multirow{2}{*}{$\begin{array}{l}\text { East (region 1) } \\
\text { flow statistic }\end{array}$} & \multirow[b]{2}{*}{ Regression model } & \multirow[b]{2}{*}{ Regression type } & \multicolumn{6}{|c|}{ Performance metrics } \\
\hline & & & Classification & SE (\%) & RMSE & Pseudo- $R^{2}$ & $\begin{array}{l}\text { Number } \\
\text { censored }\end{array}$ & $\begin{array}{c}\text { Number of } \\
\text { streamgages }\end{array}$ \\
\hline 7Q10_March & $\begin{array}{l}-0.56+0.82 \ln (C O N T D A) \\
\quad+1.78 \ln (B F I)\end{array}$ & Left censored & NA & 177.3 & 1.19 & 0.7573 & 17 & 181 \\
\hline 30Q10_March & $\begin{array}{l}-0.70+0.80 \ln (C O N T D A) \\
\quad+1.10 \ln (B F I)\end{array}$ & Left censored & NA & 153.9 & 1.10 & 0.7189 & 6 & 181 \\
\hline 1Q10_April & $\begin{array}{l}-0.83+0.81 \ln (C O N T D A) \\
\quad+1.63 \ln (B F I)\end{array}$ & Left censored & NA & 196.7 & 1.29 & 0.7204 & 19 & 181 \\
\hline 7Q10_April & $\begin{array}{l}-0.80+0.81 \ln (C O N T D A) \\
\quad+1.38 \ln (B F I)\end{array}$ & Left censored & NA & 149.3 & 1.08 & 0.7552 & 8 & 181 \\
\hline 30Q10_April & $\begin{array}{l}-0.27+0.71 \ln (C O N T D A) \\
\quad+0.57 \ln (B F I)+0.39 \ln \\
\quad(S T O R A G E)\end{array}$ & Left censored & NA & 123.5 & 0.96 & 0.6917 & 2 & 177 \\
\hline 1Q10_May & $\begin{array}{l}0.34+0.88 \ln (C O N T D A) \\
\quad+1.74 \ln (B F I)-0.45 \ln (K S A T) \\
\quad-0.20 \ln (L C 06 C R O P)\end{array}$ & Left censored & NA & 163.3 & 1.14 & 0.7478 & 15 & 181 \\
\hline 7Q10_May & $\begin{array}{l}0.37+0.90 \ln (C O N T D A) \\
\quad+1.54 \ln (B F I)-0.45 \ln (\text { KSAT }) \\
\quad-0.22 \ln (\text { LCO6CROP })\end{array}$ & Left censored & NA & 132.7 & 1.01 & 0.7791 & 11 & 181 \\
\hline 30Q10_May & $\begin{array}{l}-0.27+0.72 \ln (C O N T D A) \\
\quad+0.41 \ln (B F I)+0.40 \ln \\
\quad(\text { STORAGE })\end{array}$ & Left censored & NA & 113.7 & 0.91 & 0.7037 & 3 & 177 \\
\hline 1Q10_June & $\begin{array}{l}-0.23+0.93 \ln (C O N T D A) \\
\quad+2.17 \ln (B F I)-0.67 \ln (K S A T)\end{array}$ & Left censored & NA & 190.7 & 1.24 & 0.7654 & 24 & 181 \\
\hline 7Q10_June & $\begin{array}{l}0.01-0.90 \ln (C O N T D A) \\
\quad+1.90 \ln (B F I)-0.63 \ln (K S A T)\end{array}$ & Left censored & NA & 138.3 & 1.03 & 0.8019 & 13 & 181 \\
\hline 30Q10_June & $\begin{array}{l}0.17+0.85 \ln (C O N T D A) \\
\quad+0.86 \ln (B F I)-0.47 \ln (\text { KSAT }) \\
\quad+0.25 \ln (S T O R A G E)\end{array}$ & Left censored & NA & 80.17 & 0.71 & 0.8373 & 1 & 177 \\
\hline 1Q10_July_P ${ }_{\text {zero }}$ & $\begin{aligned} 1- & \left(e^{0.69+0.33 \ln (C O N T D A)+1.89 \ln (B F I)}\right. \\
& +0.44 \ln (L C 06 C R O P) / 1+e^{0.69+0.33 \ln } \\
& (C O N T D A)+1.89 \ln (B F I)+0.44 \ln (L C 06 C R O P))\end{aligned}$ & Logistic & 89.4 & NA & NA & NA & NA & 177 \\
\hline
\end{tabular}


[classification, percentage of time equation correctly predicts zero flow; SE, standard error; \%, percent; RMSE, root mean square error; pseudo- $R^{2}$, pseudocoefficient of determination; $1 \mathrm{Q} 10,1$-day mean flow with 10-year recurrence interval; $\mathrm{Q}$, flow; Ann, annual; $\mathrm{P}_{\text {zero }}$, probability of zero flow; $e$, base of natural logarithm, approximately equal to $2.7183 ; \ln$, natural logarithm; CONTDA, contributing drainage area; $B F I$, base-flow index; $L C 06 C R O P$, percentage of crop land cover; NA, not applicable; 7Q10, 7-day mean flow with 10-year recurrence interval; 30Q10, 30-day mean flow with 10-year recurrence interval; KSAT, saturated hydraulic conductivity; STORAGE, percentage of reservoir storage; FD, flow duration; $99.99,99.99 \%$ interval; $99.90,99.90 \%$ interval; $99,99 \%$ interval; $95,95 \%$ interval; $90,90 \%$ interval; $75,75 \%$ interval; 50, 50\% interval; 25, 25\% interval; PRECIPfull, mean annual precipitation for $1895-2017 ; 10,10 \%$ interval; 5, 5\% interval; $2,2 \%$ interval; $0.1,0.1 \%$ interval; $0.01,0.01 \%$ interval]

\begin{tabular}{|c|c|c|c|c|c|c|c|c|}
\hline \multirow{2}{*}{$\begin{array}{l}\text { East (region 1) } \\
\text { flow statistic }\end{array}$} & \multirow[b]{2}{*}{ Regression model } & \multirow[b]{2}{*}{ Regression type } & \multicolumn{6}{|c|}{ Performance metrics } \\
\hline & & & Classification & SE (\%) & RMSE & Pseudo- $R^{2}$ & $\begin{array}{l}\text { Number } \\
\text { censored }\end{array}$ & $\begin{array}{c}\text { Number of } \\
\text { streamgages }\end{array}$ \\
\hline 7Q10_July_P & 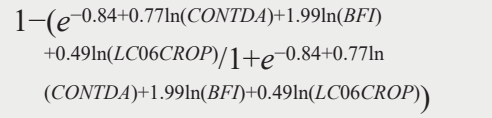 & Logistic & 97.1 & NA & NA & NA & NA & 176 \\
\hline 1Q10_July & $\begin{array}{l}0.06+1.00 \ln (C O N T D A) \\
\quad+3.64 \ln (B F I)-0.86 \ln (K S A T)\end{array}$ & Left censored & NA & 508.9 & 1.81 & 0.7362 & 67 & 181 \\
\hline 7Q10_July & $\begin{array}{l}-0.86+1.06 \ln (C O N T D A) \\
\quad+2.97 \ln (B F I)-0.80 \ln (K S A T)\end{array}$ & Left censored & NA & 427.4 & 1.72 & 0.7243 & 56 & 181 \\
\hline 30Q10_July & $\begin{array}{l}-0.61+1.01 \ln (C O N T D A) \\
\quad+1.91 \ln (B F I)-0.62 \ln (K S A T)\end{array}$ & Left censored & NA & 138.3 & 1.03 & 0.8262 & 15 & 181 \\
\hline 1Q10_August_ $\mathrm{P}_{\text {zero }}{ }^{*}$ & 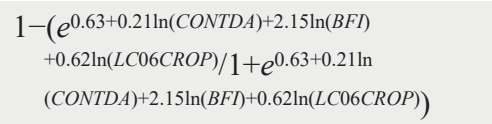 & Logistic & 82.2 & NA & NA & NA & NA & 181 \\
\hline 7Q10_August_P & $\begin{array}{l}1-\left(e^{-0.81+0.51 \ln (C O N T D A)+1.93 \ln (B F I)}\right. \\
\quad+0.62 \ln (L C 06 C R O P) / 1+e^{-0.81+0.51 \ln } \\
\quad(C O N T D A)+1.93 \ln (B F I)+0.62 \ln (L C 06 C R O P))\end{array}$ & Logistic & 88.6 & NA & NA & NA & NA & 181 \\
\hline 1Q10_August & $\begin{array}{l}-0.22+1.05 \ln (C O N T D A) \\
\quad+4.33 \ln (B F I)-0.78 \ln (K S A T)\end{array}$ & Left censored & NA & 873 & 2.08 & 0.7359 & 87 & 181 \\
\hline 7Q10_August & $\begin{array}{l}-2.48+0.79 \ln (C O N T D A) \\
\quad+2.79 \ln (B F I)+0.42 \ln (L C 06 C \mathrm{R} O P)\end{array}$ & Left censored & NA & 562.7 & 1.87 & 0.689 & 68 & 181 \\
\hline 30Q10_August & $\begin{array}{l}-1.17+1.03 \ln (C O N T D A) \\
\quad+2.33 \ln (B F I)-0.47 \ln (K S A T)\end{array}$ & Left censored & NA & 223.3 & 1.34 & 0.7667 & 31 & 177 \\
\hline 1Q10_September_P ${ }_{\text {zero }}$ & $\begin{aligned} 1- & \left(e^{0.38+0.31 \ln (C O N T D A)+2.27 \ln (B F I)}\right. \\
& +0.56 \ln (L C 06 C R O P) / 1+e^{0.38+0.31 \ln } \\
& (C O N T D A)+2.27 \ln (B F I)+0.56 \ln (L C 06 C R O P))\end{aligned}$ & Logistic & 84.8 & NA & NA & NA & NA & 181 \\
\hline 7Q10_September_P $\mathrm{z}_{\text {zero }}$ & $\begin{aligned} 1- & \left(e^{0.70+0.47 \ln (C O N T D A)+1.67 \ln (B F I) / 1}\right. \\
& \left.+e^{0.70+0.47 \ln (C O N T D A)+1.67 \ln (B F I)}\right)\end{aligned}$ & Logistic & 91.1 & NA & NA & NA & NA & 181 \\
\hline 1Q10_September & $\begin{array}{l}-0.38+0.87 \ln (C O N T D A) \\
\quad+4.32 \ln (B F I)-0.72 \ln (\text { KSAT }) \\
\quad+0.37 \ln (L C 06 C R O P)\end{array}$ & Left censored & NA & 928.4 & 2.11 & 0.726 & 84 & 181 \\
\hline 7Q10_September & $\begin{array}{l}-0.74+0.95 \ln (C O N T D A) \\
\quad+3.80 \ln (B F I)-0.77 \ln (K S A T) \\
\quad+0.32 \ln (L C 06 C R O P)\end{array}$ & Left censored & NA & 577.8 & 1.88 & 0.746 & 73 & 181 \\
\hline
\end{tabular}


Table 9. Regression equations for estimating selected low-flow frequency statistics for unregulated streams in the east region of Kansas.-Continued

[classification, percentage of time equation correctly predicts zero flow; SE, standard error; \%, percent; RMSE, root mean square error; pseudo- $R^{2}$, pseudocoefficient of determination; $1 \mathrm{Q} 10,1$-day mean flow with 10-year recurrence interval; $\mathrm{Q}$, flow; Ann, annual; $\mathrm{P}_{\text {zero }}$, probability of zero flow; $e$, base of natural logarithm, approximately equal to $2.7183 ; \ln$, natural logarithm; CONTDA, contributing drainage area; $B F I$, base-flow index; $L C 06 C R O P$, percentage of crop land cover; NA, not applicable; 7Q10, 7-day mean flow with 10-year recurrence interval; 30Q10, 30-day mean flow with 10-year recurrence interval; KSAT, saturated hydraulic conductivity; STORAGE, percentage of reservoir storage; FD, flow duration; $99.99,99.99 \%$ interval; $99.90,99.90 \%$ interval; $99,99 \%$ interval; $95,95 \%$ interval; $90,90 \%$ interval; $75,75 \%$ interval; 50, 50\% interval; 25, 25\% interval; PRECIPfull, mean annual precipitation for $1895-2017 ; 10,10 \%$ interval; 5, 5\% interval; $2,2 \%$ interval; $0.1,0.1 \%$ interval; $0.01,0.01 \%$ interval $]$

\begin{tabular}{|c|c|c|c|c|c|c|c|c|}
\hline \multirow{2}{*}{$\begin{array}{l}\text { East (region 1) } \\
\text { flow statistic }\end{array}$} & \multirow[b]{2}{*}{ Regression model } & \multirow[b]{2}{*}{ Regression type } & \multicolumn{6}{|c|}{ Performance metrics } \\
\hline & & & Classification & SE (\%) & RMSE & Pseudo- $R^{2}$ & $\begin{array}{l}\text { Number } \\
\text { censored }\end{array}$ & $\begin{array}{c}\text { Number of } \\
\text { streamgages }\end{array}$ \\
\hline 30Q10_September & $\begin{array}{l}-1.00+1.06 \ln (C O N T D A) \\
\quad+2.62 \ln (B F I)-0.60 \ln (K S A T)\end{array}$ & Left censored & NA & 266.6 & 1.45 & 0.7561 & 37 & 177 \\
\hline 1Q10_October_P $\mathrm{zero}{ }^{*}$ & $\begin{aligned} 1- & \left(e^{0.33+0.27 \ln (C O N T D A)+2.10 \ln (B F I)}\right. \\
& +0.60 \ln (L C 06 C R O P) / 1+e^{0.33+0.27 \ln } \\
& (C O N T D A)+2.10 \ln (B F I++0.60 \ln (L C 06 C R O P))\end{aligned}$ & Logistic & 82.4 & NA & NA & NA & NA & 181 \\
\hline 7Q10_October_P ${ }_{\text {zero }}$ & $\begin{aligned} 1- & \left(e^{0.48+0.33 \ln (C O N T D A)+2.08 \ln (B F I)}\right. \\
& +0.56 \ln (L C 06 C R O P) / 1+e^{0.48+0.33 \ln } \\
& (C O N T D A)+2.08 \ln (B F I+0.56 \ln (L C 06 C R O P))\end{aligned}$ & Logistic & 87.4 & NA & NA & NA & NA & 181 \\
\hline 1Q10_October & $\begin{array}{l}0.17+0.74 \ln (C O N T D A) \\
\quad+4.44 \ln (B F I)-0.61 \ln (K S A T) \\
\quad+0.53 \ln (L C 06 C R O P)\end{array}$ & Left censored & NA & 756 & 2.02 & 0.7462 & 82 & 181 \\
\hline 7Q10_October & $\begin{array}{l}-0.05+0.83 \ln (C O N T D A) \\
\quad+4.12 \ln (B F I)-0.72 \ln (K S A T) \\
\quad+0.47 \ln (L C 06 C R O P)\end{array}$ & Left censored & NA & 573.8 & 1.88 & 0.7589 & 74 & 181 \\
\hline 30Q10_October & $\begin{array}{l}0.37+0.78 \ln (C O N T D A) \\
\quad+3.36 \ln (B F I)-0.55 \ln (K S A T) \\
\quad+0.32 \ln (L C 06 C R O P)\end{array}$ & Left censored & NA & 320.8 & 1.56 & 0.7683 & 51 & 181 \\
\hline 1Q10_November_P ${ }_{\text {zero }} *$ & $\begin{aligned} 1- & \left(e^{2.56+0.20 \ln (C O N T D A)+2.53 \ln (B F I)}\right. \\
& +0.53 \ln (L C 06 C R O P) / 1+e^{2.56+0.20 \ln } \\
& (C O N T D A)+2.53 \ln (B F I)+0.53 \ln (L C 06 C R O P))\end{aligned}$ & Logistic & 92.4 & NA & NA & NA & NA & 181 \\
\hline 7Q10_November_P zero & $\begin{aligned} 1- & \left(e^{1.47+0.43 \ln (C O N T D A)+2.27 \ln (B F I)}\right. \\
& +0.43 \ln (L C 06 C R O P) / 1+e^{1.47+0.43 \ln } \\
& (C O N T D A)+2.27 \ln (B F I)+0.43 \ln (L C O 6 C R O P))\end{aligned}$ & Logistic & 94.3 & NA & NA & NA & NA & 181 \\
\hline 1Q10_November & $\begin{array}{l}0.27+0.54 \ln (C O N T D A) \\
\quad+3.31 \ln (B F I)+0.43 \ln \\
\quad(L C 06 C R O P)\end{array}$ & Left censored & NA & 377.6 & 1.65 & 0.7328 & 61 & 181 \\
\hline 7Q10_November & $\begin{array}{l}0.38+0.57 \ln (C O N T D A) \\
\quad+3.29 \ln (B F I)+0.43 \ln \\
\quad(L C 06 C R O P)\end{array}$ & Left censored & NA & 334.3 & 1.58 & 0.754 & 55 & 181 \\
\hline 30Q10_November & $\begin{array}{l}-0.69+0.73 \ln (C O N T D A) \\
\quad+2.42 \ln (B F I)+0.28 \ln \\
\quad(L C 06 C R O P)\end{array}$ & Left censored & NA & 242.3 & 1.39 & 0.7536 & 31 & 181 \\
\hline
\end{tabular}


[classification, percentage of time equation correctly predicts zero flow; SE, standard error; \%, percent; RMSE, root mean square error; pseudo- $R^{2}$, pseudocoefficient of determination; $1 \mathrm{Q} 10,1$-day mean flow with 10-year recurrence interval; $\mathrm{Q}$, flow; Ann, annual; $\mathrm{P}_{\text {zero }}$, probability of zero flow; $e$, base of natural logarithm, approximately equal to $2.7183 ; \ln$, natural logarithm; CONTDA, contributing drainage area; $B F I$, base-flow index; $\angle C 06 C R O P$, percentage of crop land cover; NA, not applicable; 7Q10, 7-day mean flow with 10-year recurrence interval; 30Q10, 30-day mean flow with 10-year recurrence interval; KSAT, saturated hydraulic conductivity; STORAGE, percentage of reservoir storage; FD, flow duration; $99.99,99.99 \%$ interval; $99.90,99.90 \%$ interval; $99,99 \%$ interval; $95,95 \%$ interval; $90,90 \%$ interval; 75, 75\% interval; 50, 50\% interval; 25, 25\% interval; PRECIPfull, mean annual precipitation for $1895-2017 ; 10,10 \%$ interval; 5, 5\% interval; $2,2 \%$ interval; $0.1,0.1 \%$ interval; $0.01,0.01 \%$ interval]

\begin{tabular}{|c|c|c|c|c|c|c|c|c|}
\hline \multirow{2}{*}{$\begin{array}{l}\text { East (region 1) } \\
\text { flow statistic }\end{array}$} & \multirow[b]{2}{*}{ Regression model } & \multirow[b]{2}{*}{ Regression type } & \multicolumn{6}{|c|}{ Performance metrics } \\
\hline & & & Classification & SE (\%) & RMSE & Pseudo- $R^{2}$ & $\begin{array}{l}\text { Number } \\
\text { censored }\end{array}$ & $\begin{array}{c}\text { Number of } \\
\text { streamgages }\end{array}$ \\
\hline 1Q10_December & $\begin{array}{l}-0.38+0.68 \ln (C O N T D A) \\
\quad+2.62 \ln (B F I)+0.21 \ln \\
\quad(L C 06 C R O P)\end{array}$ & Left censored & NA & 221.1 & 1.33 & 0.7696 & 41 & 181 \\
\hline 7Q10_December & $\begin{array}{l}-0.66+0.72 \ln (C O N T D A) \\
\quad+2.52 \ln (B F I)+0.25 \ln \\
\quad(L C 06 C R O P)\end{array}$ & Left censored & NA & 224.5 & 1.34 & 0.7704 & 37 & 181 \\
\hline 30Q10_December & $\begin{array}{l}-0.92+0.79 \ln (C O N T D A) \\
\quad+2.09 \ln (B F I)+0.15 \ln \\
\quad(L C 06 C R O P)\end{array}$ & Left censored & NA & 194.4 & 1.25 & 0.7537 & 22 & 175 \\
\hline FD_99.99_P & $\begin{array}{c}1-\left(e^{-0.13+0.30 \ln (C O N T D A)+1.89 \ln (B F I) / 1}\right. \\
\left.+e^{-0.13+0.30 \ln (C O N T D A)+1.89 \ln (B F I)}\right)\end{array}$ & Logistic & 56.4 & NA & NA & NA & NA & 181 \\
\hline FD_99.90_P zero ${ }^{*}$ & 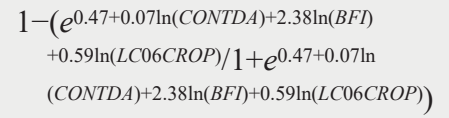 & Logistic & 69.2 & NA & NA & NA & NA & 181 \\
\hline FD_99_P zero & $\begin{array}{c}1-\left(e^{1.69+0.28 \ln (C O N T D A)+2.01 \ln (B F I) / 1}\right. \\
\left.+e^{1.69+0.28 \ln (C O N T D A)+2.01 \ln (B F I)}\right)\end{array}$ & Logistic & 78.6 & NA & NA & NA & NA & 181 \\
\hline FD_95_P zero & 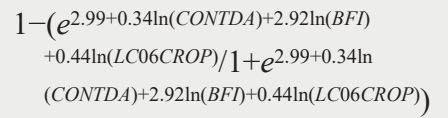 & Logistic & 94.2 & NA & NA & NA & NA & 181 \\
\hline FD_99.99 & $\begin{array}{l}-3.35+0.99 \ln (C O N T D A) \\
\quad+5.05 \ln (B F I)\end{array}$ & Left censored & NA & 13,270 & 3.13 & 0.6274 & 130 & 181 \\
\hline FD_99.90 & $\begin{array}{l}-0.43+1.09 \ln (C O N T D A) \\
\quad+6.24 \ln (B F I)-0.86 \ln (K S A T)\end{array}$ & Left censored & NA & 6,074 & 2.87 & 0.6925 & 116 & 176 \\
\hline FD_99 & $\begin{array}{l}-1.44+0.66 \ln (C O N T D A) \\
\quad+3.76 \ln (B F I)+0.58 \ln \\
\quad(L C 06 C R O P)\end{array}$ & Left censored & NA & 1,079 & 2.18 & 0.6845 & 83 & 181 \\
\hline FD_95 & $\begin{array}{l}-1.46+0.75 \ln (C O N T D A) \\
\quad+2.73 \ln (B F I)+0.36 \ln \\
\quad(L C 06 C R O P)\end{array}$ & Left censored & NA & 372.5 & 1.64 & 0.7241 & 53 & 181 \\
\hline FD_90 & $\begin{array}{l}-0.36+0.85 \ln (C O N T D A) \\
\quad+2.61 \ln (B F I)-0.39 \ln (K S A T) \\
\quad+0.20 \ln (L C 06 C R O P)\end{array}$ & Left censored & NA & 229.4 & 1.35 & 0.754 & 30 & 175 \\
\hline
\end{tabular}


Table 9. Regression equations for estimating selected low-flow frequency statistics for unregulated streams in the east region of Kansas.-Continued

[classification, percentage of time equation correctly predicts zero flow; SE, standard error; \%, percent; RMSE, root mean square error; pseudo- $R^{2}$, pseudocoefficient of determination; $1 \mathrm{Q} 10,1$-day mean flow with 10-year recurrence interval; $\mathrm{Q}$, flow; Ann, annual; $\mathrm{P}_{\text {zero }}$, probability of zero flow; $e$, base of natural logarithm, approximately equal to $2.7183 ; 1 \mathrm{n}$, natural logarithm; CONTDA, contributing drainage area; $B F I$, base-flow index; $L C 06 C R O P$, percentage of crop land cover; NA, not applicable; 7Q10, 7-day mean flow with 10-year recurrence interval; 30Q10, 30-day mean flow with 10-year recurrence interval; KSAT, saturated hydraulic conductivity; STORAGE, percentage of reservoir storage; FD, flow duration; 99.99, 99.99\% interval; 99.90, 99.90\% interval; 99, 99\% interval; 95, 95\% interval; 90, 90\% interval; $75,75 \%$ interval; $50,50 \%$ interval; $25,25 \%$ interval; PRECIPfull, mean annual precipitation for $1895-2017 ; 10,10 \%$ interval; $5,5 \%$ interval; $2,2 \%$ interval; $0.1,0.1 \%$ interval; $0.01,0.01 \%$ interval $]$

\begin{tabular}{|c|c|c|c|c|c|c|c|c|}
\hline \multirow{2}{*}{$\begin{array}{l}\text { East (region 1) } \\
\text { flow statistic }\end{array}$} & \multirow[b]{2}{*}{ Regression model } & \multirow[b]{2}{*}{ Regression type } & \multicolumn{6}{|c|}{ Performance metrics } \\
\hline & & & Classification & SE (\%) & RMSE & Pseudo- $R^{2}$ & $\begin{array}{l}\text { Number } \\
\text { censored }\end{array}$ & $\begin{array}{c}\text { Number of } \\
\text { streamgages }\end{array}$ \\
\hline FD_75 & $\begin{array}{l}0.36+0.82 \ln (C O N T D A) \\
\quad+1.65 \ln (B F I)-0.33 \ln (K S A T)\end{array}$ & Left censored & NA & 93.43 & 0.79 & 0.8447 & 2 & 176 \\
\hline FD_50 & $\begin{array}{l}1.22+0.77 \ln (C O N T D A) \\
\quad+1.06 \ln (B F I)-0.46 \ln (K S A T) \\
\quad+0.21 \ln (S T O R A G E)\end{array}$ & Left censored & NA & 73.63 & 0.66 & 0.8557 & 1 & 181 \\
\hline FD_25 & $\begin{array}{c}-16.20+0.94 \ln (C O N T D A) \\
\quad+4.27 \ln (\text { PRECIPfull })\end{array}$ & Left censored & NA & 55.17 & 0.52 & 0.8953 & 0 & 181 \\
\hline FD_10 & $\begin{array}{c}-16.12+0.90 \ln (\text { CONTDA }) \\
\quad+0.11 \ln (\text { STORAGE }) \\
\quad+4.61 \ln (\text { PRECIPfull })\end{array}$ & Left censored & NA & 39.62 & 0.36 & 0.9395 & 0 & 181 \\
\hline FD_5 & $\begin{array}{c}-15.09+0.86 \ln (\text { CONTDA }) \\
+0.15 \ln (\text { STORAGE }) \\
+4.61 \ln (\text { PRECIPfull })\end{array}$ & Left censored & NA & 36.93 & 0.36 & 0.9432 & 0 & 181 \\
\hline FD_2 & $\begin{array}{l}-10.19+0.70 \ln (\text { CONTDA }) \\
\quad+0.08 \ln (\text { STORAGE }) \\
\quad+3.91 \ln (\text { PRECIPfull })\end{array}$ & Left censored & NA & 37.41 & 0.36 & 0.9116 & 0 & 177 \\
\hline FD_0.1 & $\begin{array}{l}-6.24+0.64 \ln (\text { CONTDA }) \\
\quad+3.22 \ln (\text { PRECIPfull })\end{array}$ & Left censored & NA & 38.21 & 0.37 & 0.8867 & 0 & 181 \\
\hline FD_0.01 & $\begin{array}{l}-3.70+0.58 \ln (\text { CONTDA }) \\
\quad+0.11 \ln (\text { STORAGE }) \\
\quad+2.80 \ln (\text { PRECIPfull })\end{array}$ & Left censored & NA & 42.58 & 0.41 & 0.8456 & 0 & 178 \\
\hline Mean_Ann_Q & $\begin{array}{l}-14.2+0.82 \ln (\text { CONTDA }) \\
\quad+4.08 \ln (\text { PRECIPfull })\end{array}$ & Left censored & NA & 33.19 & 0.32 & 0.944 & 0 & 181 \\
\hline
\end{tabular}

*CONTDA was not statistically significant but was included in the model. 
[classification, percentage of time equation correctly predicts zero flow; SE, standard error; \%, percent; RMSE, root mean square error; pseudo- $R^{2}$, pseudocoefficient of determination; 1Q10, 1-day mean flow with 10-year recurrence interval; $\mathrm{Q}$, flow; Ann, annual; $\mathrm{P}_{\text {zero }}$, probability of zero flow; $e$, base of natural logarithm, approximately equal to 2.7183 ; ln, natural logarithm; CONTDA, contributing drainage area; $B F I$, base-flow index; SLOPE, mean basin slope; NA, not applicable; 7Q10, 7-day mean flow with 10-year recurrence interval; 30Q10, 30-day mean flow with 10-year recurrence interval; KSAT, saturated hydraulic conductivity; FD, flow duration; $99.99,99.99 \%$ interval; $99.90,99.90 \%$ interval; $99,99 \%$ interval; $95,95 \%$ interval; $90,90 \%$ interval; $75,75 \%$ interval; $50,50 \%$ interval; $25,25 \%$ interval; $10,10 \%$ interval; 5, 5\% interval; PRECIPfull, mean annual precipitation for $1895-2017 ; 2,2 \%$ interval; $0.1,0.1 \%$ interval; $0.01,0.01 \%$ interval]

\begin{tabular}{|c|c|c|c|c|c|c|c|c|}
\hline \multirow[b]{2}{*}{$\begin{array}{l}\text { West (region 2) } \\
\text { flow statistic }\end{array}$} & \multirow[b]{2}{*}{ Regression model } & \multirow[b]{2}{*}{ Regression type } & \multicolumn{6}{|c|}{ Performance metrics } \\
\hline & & & Classification & SE (\%) & RMSE & Pseudo- $R^{2}$ & $\begin{array}{l}\text { Number } \\
\text { censored }\end{array}$ & $\begin{array}{c}\text { Number of } \\
\text { streamgages }\end{array}$ \\
\hline 1Q10_Ann_P zero $*$ & $\begin{array}{c}1-\left(e^{-2.09+0.10 \ln (C O N T D A)+5.08 \ln (B F I)}\right. \\
\quad+3.75 \ln (S L O P E) / 1+e^{-2.09+0.10 \ln } \\
\quad(C O N T D A)+5.08 \ln (B F I)+3.75 \ln (S L O P E))\end{array}$ & Logistic & 62.5 & NA & NA & NA & NA & 71 \\
\hline 7Q10_Ann_P zero $*$ & $\begin{array}{c}1-\left(e^{-1.12+0.16 \ln (C O N T D A)+3.43 \ln (B F I)}\right. \\
+2.08 \ln (S L O P E) / 1+e^{-1.12+0.16 \ln } \\
(C O N T D A)+3.43 \ln (B F I)+2.08 \ln (S L O P E))\end{array}$ & Logistic & 63.6 & NA & NA & NA & NA & 73 \\
\hline 30Q10_Ann_P ${ }_{\text {zero }} *$ & $\begin{array}{c}1-\left(e^{1.38+0.17 \ln (C O N T D A)+3.24 \ln (B F I) / 1}\right. \\
\left.+e^{1.38+0.17 \ln (C O N T D A)+3.24 \ln (B F I)}\right)\end{array}$ & Logistic & 65.4 & NA & NA & NA & NA & 73 \\
\hline 1Q10_Ann* & $\begin{array}{l}1.76-0.52 \ln (C O N T D A) \\
\quad+7.56 \ln (B F I)+2.32 \ln (S L O P E)\end{array}$ & Left censored & NA & 2,649 & 2.56 & 0.819 & 53 & 66 \\
\hline 7Q10_Ann* & $\begin{array}{l}0.40-0.36 \ln (C O N T D A) \\
\quad+6.43 \ln (B F I)+2.44 \ln (S L O P E)\end{array}$ & Left censored & NA & 3,022 & 2.61 & 0.7652 & 49 & 66 \\
\hline 30Q10_Ann* & $\begin{array}{l}-1.44+0.001 \ln (C O N T D A) \\
\quad+5.34 \ln (B F I)+2.20 \ln (S L O P E)\end{array}$ & Left censored & NA & 3,581 & 2.68 & 0.8348 & 46 & 68 \\
\hline 1Q10_January_P ${ }_{\text {zero }} *$ & $\begin{array}{c}1-\left(e^{5.07+0.17 \ln (C O N T D A)+5.08 \ln (B F) / 1}\right. \\
\left.+e^{5.07+0.17 \ln (C O N T D A)+5.08 \ln (B F I)}\right)\end{array}$ & Logistic & 92.9 & NA & NA & NA & NA & 72 \\
\hline 7Q10_January_P ${ }_{\text {zero }} *$ & $\begin{array}{c}1-\left(e^{3.22+0.38 \ln (C O N T D A)+4.65 \ln (B F I) / 1}\right. \\
\left.\quad+e^{3.22+0.38 \ln (C O N T D A)+4.65 \ln (B F I)}\right)\end{array}$ & Logistic & 88.4 & NA & NA & NA & NA & 73 \\
\hline 30Q10_January_P ${ }_{\text {zero }} *$ & $\begin{array}{c}1-\left(e^{2.60+0.46 \ln (C O N T D A)+4.25 \ln (B F I) / 1}\right. \\
\left.+e^{2.60+0.46 \ln (C O N T D A)+4.25 \ln (B F I)}\right)\end{array}$ & Logistic & 89.1 & NA & NA & NA & NA & 73 \\
\hline 1Q10_January & $\begin{array}{l}-2.68+0.53 \ln (C O N T D A) \\
\quad+4.88 \ln (B F I)+0.88 \ln (K S A T)\end{array}$ & Left censored & NA & 418.8 & 1.71 & 0.9105 & 34 & 73 \\
\hline 7Q10_January & $\begin{array}{l}-2.50+0.54 \ln (C O N T D A) \\
\quad+4.84 \ln (B F I)+0.92 \ln (K S A T)\end{array}$ & Left censored & NA & 395.4 & 1.68 & 0.9126 & 31 & 73 \\
\hline 30Q10_January & $\begin{array}{l}-3.47+0.63 \ln (C O N T D A) \\
\quad+4.69 \ln (B F I)+1.16 \ln (K S A T)\end{array}$ & Left censored & NA & 590.1 & 1.89 & 0.8897 & 29 & 73 \\
\hline 1Q10_February_P $\mathrm{zero} *$ & $\begin{array}{c}1-\left(e^{5.23+0.19 \ln (C O N T D A)+5.12 \ln (B F I) / 1}\right. \\
\left.+e^{5.23+0.19 \ln (C O N T D A)+5.12 \ln (B F I)}\right)\end{array}$ & Logistic & 90.9 & NA & NA & NA & NA & 73 \\
\hline 7Q10_February_P ${ }_{\text {zero }}$ & $\begin{array}{c}1-\left(e^{1.40+0.65 \ln (C O N T D A)+3.94 \ln (B F I) / 1}\right. \\
\left.+e^{1.40+0.65 \ln (C O N T D A)+3.94 \ln (B F I)}\right)\end{array}$ & Logistic & 93.9 & NA & NA & NA & NA & 73 \\
\hline
\end{tabular}


Table 10. Regression equations for estimating selected low-flow frequency statistics for unregulated streams in the west region of Kansas.-Continued

[classification, percentage of time equation correctly predicts zero flow; SE, standard error; \%, percent; RMSE, root mean square error; pseudo- $R^{2}$, pseudocoefficient of determination; $1 \mathrm{Q} 10,1$-day mean flow with 10-year recurrence interval; $\mathrm{Q}$, flow; Ann, annual; $\mathrm{P}_{\text {zero }}$, probability of zero flow; $e$, base of natural logarithm, approximately equal to $2.7183 ; \ln$, natural logarithm; CONTDA, contributing drainage area; $B F I$, base-flow index; SLOPE, mean basin slope; NA, not applicable; 7Q10, 7-day mean flow with 10-year recurrence interval; 30Q10, 30-day mean flow with 10-year recurrence interval; KSAT, saturated hydraulic conductivity; FD, flow duration; $99.99,99.99 \%$ interval; $99.90,99.90 \%$ interval; $99,99 \%$ interval; $95,95 \%$ interval; $90,90 \%$ interval; $75,75 \%$ interval; $50,50 \%$ interval; $25,25 \%$ interval; $10,10 \%$ interval; 5, 5\% interval; PRECIPfull, mean annual precipitation for $1895-2017 ; 2,2 \%$ interval; $0.1,0.1 \%$ interval; $0.01,0.01 \%$ interval]

\begin{tabular}{|c|c|c|c|c|c|c|c|c|}
\hline \multirow{2}{*}{$\begin{array}{l}\text { West (region 2) } \\
\text { flow statistic }\end{array}$} & \multirow[b]{2}{*}{ Regression model } & \multirow[b]{2}{*}{ Regression type } & \multicolumn{6}{|c|}{ Performance metrics } \\
\hline & & & Classification & SE (\%) & RMSE & Pseudo- $R^{2}$ & $\begin{array}{l}\text { Number } \\
\text { censored }\end{array}$ & $\begin{array}{c}\text { Number of } \\
\text { streamgages }\end{array}$ \\
\hline 30Q10_February_P zero ${ }^{*}$ & $\begin{array}{c}1-\left(e^{3.94+0.56 \ln (C O N T D A)+4.92 \ln (B F I) / 1}\right. \\
\left.+e^{3.94+0.56 \ln (C O N T D A)+4.92 \ln (B F I)}\right)\end{array}$ & Logistic & 96.2 & NA & NA & NA & NA & 73 \\
\hline 1Q10_February & $\begin{array}{l}-2.97+0.56 \ln (C O N T D A) \\
\quad+4.57 \ln (B F I)+0.98 \ln (K S A T)\end{array}$ & Left censored & NA & 451.9 & 1.75 & 0.8974 & 31 & 73 \\
\hline 7Q10_February & $\begin{array}{l}-3.53+0.61 \ln (C O N T D A) \\
\quad+4.53 \ln (B F I)+1.17 \ln (K S A T)\end{array}$ & Left censored & NA & 493 & 1.8 & 0.8934 & 29 & 73 \\
\hline 30Q10_February & $\begin{array}{l}-2.44+0.58 \ln (C O N T D A) \\
\quad+4.02 \ln (B F I)+0.93 \ln (K S A T)\end{array}$ & Left censored & NA & 596.4 & 1.9 & 0.8551 & 23 & 73 \\
\hline 1Q10_March_P ${ }_{\text {zero }} *$ & $\begin{array}{c}1-\left(e^{5.06+0.14 \ln (C O N T D A)+4.16 \ln (B F I) / 1}\right. \\
\left.+e^{5.06+0.14 \ln (C O N T D A)+4.16 \ln (B F I)}\right)\end{array}$ & Logistic & 89.8 & NA & NA & NA & NA & 73 \\
\hline 7Q10_March_P zero & $\begin{array}{c}1-\left(e^{3.94+0.56 \ln (C O N T D A)+4.92 \ln (B F I) / 1}\right. \\
\left.+e^{3.94+0.56 \ln (C O N T D A)+4.92 \ln (B F I)}\right)\end{array}$ & Logistic & 96.2 & NA & NA & NA & NA & 73 \\
\hline 30Q10_March_P ${ }_{\text {zero }}{ }^{*}$ & $\begin{array}{c}1-\left(e^{3.68+0.56 \ln (C O N T D A)+4.60 \ln (B F I) / 1}\right. \\
\left.+e^{3.68+0.56 \ln (C O N T D A)+4.60 \ln (B F I)}\right)\end{array}$ & Logistic & 94.4 & NA & NA & NA & NA & 73 \\
\hline 1Q10_March & $\begin{array}{l}0.96+0.48 \ln (C O N T D A) \\
\quad+4.77 \ln (B F I)\end{array}$ & Left censored & NA & 373.2 & 1.64 & 0.9053 & 25 & 73 \\
\hline 7Q10_March & $\begin{array}{l}1.11+0.49 \ln (C O N T D A) \\
\quad+4.74 \ln (B F I)\end{array}$ & Left censored & NA & 528.2 & 1.83 & 0.884 & 24 & 73 \\
\hline 30Q10_March & $\begin{array}{l}0.60+0.63 \ln (C O N T D A) \\
\quad+4.69 \ln (B F I)\end{array}$ & Left censored & NA & 505.2 & 1.81 & 0.8866 & 21 & 73 \\
\hline 1Q10_April_P ${ }_{\text {zero }} *$ & $\begin{array}{c}1-\left(e^{4.21+0.25 \ln (C O N T D A)+3.92 \ln (B F I) / 1}\right. \\
\left.+\quad e^{4.21+0.25 \ln (C O N T D A)+3.92 \ln (B F I)}\right)\end{array}$ & Logistic & 94.0 & NA & NA & NA & NA & 72 \\
\hline 7Q10_April_P ${ }_{\text {zero }} *$ & $\begin{array}{c}1-\left(e^{4.64+0.40 \ln (C O N T D A)+4.79 \ln (B F I) / 1}\right. \\
\left.+\quad e^{4.64+0.40 \ln (C O N T D A)+4.79 \ln (B F I)}\right)\end{array}$ & Logistic & 96.1 & NA & NA & NA & NA & 71 \\
\hline 30Q10_April_P ${ }_{\text {zero }} *$ & $\begin{array}{c}1-\left(e^{3.12+0.49 \ln (C O N T D A)+3.87 \ln (B F I) / 1}\right. \\
\left.+e^{3.12+0.49 \ln (C O N T D A)+3.87 \ln (B F I)}\right)\end{array}$ & Logistic & 98.1 & NA & NA & NA & NA & 71 \\
\hline 1Q10_April & $\begin{array}{l}0.74+0.55 \ln (C O N T D A) \\
\quad+5.22 \ln (B F I)\end{array}$ & Left censored & NA & 356.7 & 1.62 & 0.9223 & 26 & 73 \\
\hline 7Q10_April & $\begin{array}{l}1.13+0.49 \ln (C O N T D A) \\
\quad+4.74 \ln (B F I)\end{array}$ & Left censored & NA & 376 & 1.65 & 0.9041 & 23 & 73 \\
\hline
\end{tabular}


[classification, percentage of time equation correctly predicts zero flow; SE, standard error; \%, percent; RMSE, root mean square error; pseudo- $R^{2}$, pseudocoefficient of determination; 1Q10, 1-day mean flow with 10-year recurrence interval; $\mathrm{Q}$, flow; Ann, annual; $\mathrm{P}_{\text {zero }}$, probability of zero flow; $e$, base of natural logarithm, approximately equal to 2.7183 ; ln, natural logarithm; CONTDA, contributing drainage area; $B F I$, base-flow index; SLOPE, mean basin slope; NA, not applicable; 7Q10, 7-day mean flow with 10-year recurrence interval; 30Q10, 30-day mean flow with 10-year recurrence interval; KSAT, saturated hydraulic conductivity; FD, flow duration; $99.99,99.99 \%$ interval; $99.90,99.90 \%$ interval; $99,99 \%$ interval; $95,95 \%$ interval; $90,90 \%$ interval; $75,75 \%$ interval; 50, 50\% interval; $25,25 \%$ interval; $10,10 \%$ interval; 5, 5\% interval; PRECIPfull, mean annual precipitation for $1895-2017 ; 2,2 \%$ interval; $0.1,0.1 \%$ interval; $0.01,0.01 \%$ interval]

\begin{tabular}{|c|c|c|c|c|c|c|c|c|}
\hline \multirow{2}{*}{$\begin{array}{l}\text { West (region 2) } \\
\text { flow statistic }\end{array}$} & \multirow[b]{2}{*}{ Regression model } & \multirow[b]{2}{*}{ Regression type } & \multicolumn{6}{|c|}{ Performance metrics } \\
\hline & & & Classification & SE (\%) & RMSE & Pseudo- $R^{2}$ & $\begin{array}{l}\text { Number } \\
\text { censored }\end{array}$ & $\begin{array}{c}\text { Number of } \\
\text { streamgages }\end{array}$ \\
\hline 30Q10_April & $\begin{array}{l}0.89+0.56 \ln (C O N T D A) \\
\quad+4.42 \ln (B F I)\end{array}$ & Left censored & NA & 342 & 1.59 & 0.8994 & 20 & 73 \\
\hline 1Q10_May_P zero ${ }^{*}$ & $\begin{array}{c}1-\left(e^{11.05-0.43 \ln (C O N T D A)+6.75 \ln (B F I) / 1}\right. \\
\left.+e^{11.05-0.43 \ln (C O N T D A)+6.75 \ln (B F I)}\right)\end{array}$ & Logistic & 90.2 & NA & NA & NA & NA & 71 \\
\hline 7Q10_May_P zero ${ }^{*}$ & $\begin{array}{c}1-\left(e^{4.85+0.21 \ln (C O N T D A)+4.46 \ln (B F I) / 1}\right. \\
\left.+\quad e^{4.85+0.21 \ln (C O N T D A)+4.46 \ln (B F I)}\right)\end{array}$ & Logistic & 87.2 & NA & NA & NA & NA & 71 \\
\hline 1Q10_May & $\begin{array}{l}3.86+0.20 \ln (C O N T D A) \\
\quad+5.94 \ln (B F I)-0.42 \ln (\text { KSAT })\end{array}$ & Left censored & NA & 487.6 & 1.79 & 0.829 & 29 & 69 \\
\hline 7Q10_May & $\begin{array}{l}2.74+0.18 \ln (C O N T D A) \\
\quad+4.94 \ln (B F I)\end{array}$ & Left censored & NA & 359.8 & 1.62 & 0.8367 & 25 & 70 \\
\hline 30Q10_May & $\begin{array}{l}0.48+0.57 \ln (C O N T D A) \\
\quad+3.86 \ln (B F I)\end{array}$ & Left censored & NA & 236.5 & 1.4 & 0.9021 & 16 & 73 \\
\hline 1Q10_June_P $\mathrm{P}_{\text {zero }} *$ & $\begin{array}{c}1-\left(e^{2.87+0.19 \ln (C O N T D A)+4.49 \ln (B F I) / 1}\right. \\
\left.+\quad e^{2.87+0.19 \ln (C O N T D A)+4.49 \ln (B F I)}\right)\end{array}$ & Logistic & 80.6 & NA & NA & NA & NA & 73 \\
\hline 7Q10_June_P zero $^{*}$ & $\begin{aligned} 1- & \left(e^{4.90+0.02 \ln (C O N T D A)+3.79 \ln (B F I) / 1}\right. \\
& \left.+e^{4.90+0.02 \ln (C O N T D A)+3.79 \ln (B F I)}\right)\end{aligned}$ & Logistic & 88.9 & NA & NA & NA & NA & 73 \\
\hline 1Q10_June* & $\begin{array}{l}2.11+0.17 \ln (C O N T D A) \\
\quad+6.90 \ln (B F I)\end{array}$ & Left censored & NA & 2,762 & 2.58 & 0.804 & 40 & 67 \\
\hline 7Q10_June* & $\begin{array}{l}1.50+0.15 \ln (C O N T D A) \\
\quad+4.33 \ln (B F I)\end{array}$ & Left censored & NA & 818.6 & 2.05 & 0.7108 & 30 & 70 \\
\hline 30Q10_June & $\begin{array}{l}-0.93+0.66 \ln (C O N T D A) \\
\quad+3.00 \ln (B F I)\end{array}$ & Left censored & NA & 294.6 & 1.51 & 0.8344 & 13 & 73 \\
\hline 1Q10_July_P ${ }_{\text {zero }} *$ & $\begin{array}{c}1-\left(e^{0.006-0.01 \ln (C O N T D A)+3.67 \ln (B F I)}\right. \\
+2.49 \ln (S L O P E) / 1+e^{0.006-0.01 \ln } \\
\quad(C O N T D A)+3.67 \ln (B F I)+2.49 \ln (S L O P E))\end{array}$ & Logistic & 72.0 & NA & NA & NA & NA & 71 \\
\hline 7Q10_July_P zero $*$ & $\begin{array}{c}1-\left(e^{2.94-0.02 \ln (C O N T D A)+2.94 \ln (B F I) / 1}\right. \\
\left.\quad+e^{2.94-0.02 \ln (C O N T D A)+2.94 \ln (B F I)}\right)\end{array}$ & Logistic & 75.0 & NA & NA & NA & NA & 73 \\
\hline 30Q10_July_P ${ }_{\text {zero }}$ * & 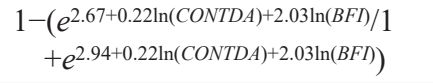 & Logistic & 96.5 & NA & NA & NA & NA & 73 \\
\hline 1Q10_July* & $\begin{array}{l}-3.21+0.17 \ln (C O N T D A) \\
\quad+6.34 \ln (B F I)+2.63 \ln (S L O P E)\end{array}$ & Left censored & NA & 22,090 & 3.29 & 0.661 & 48 & 65 \\
\hline
\end{tabular}


Table 10. Regression equations for estimating selected low-flow frequency statistics for unregulated streams in the west region of Kansas.-Continued

[classification, percentage of time equation correctly predicts zero flow; SE, standard error; \%, percent; RMSE, root mean square error; pseudo- $R^{2}$, pseudocoefficient of determination; $1 \mathrm{Q} 10,1$-day mean flow with 10-year recurrence interval; $\mathrm{Q}$, flow; Ann, annual; $\mathrm{P}_{\text {zero }}$, probability of zero flow; $e$, base of natural logarithm, approximately equal to $2.7183 ;$ ln, natural logarithm; CONTDA, contributing drainage area; $B F I$, base-flow index; SLOPE, mean basin slope; NA, not applicable; 7Q10, 7-day mean flow with 10-year recurrence interval; 30Q10, 30-day mean flow with 10-year recurrence interval; KSAT, saturated hydraulic conductivity; FD, flow duration; $99.99,99.99 \%$ interval; $99.90,99.90 \%$ interval; $99,99 \%$ interval; $95,95 \%$ interval; $90,90 \%$ interval; $75,75 \%$ interval; $50,50 \%$ interval; $25,25 \%$ interval; $10,10 \%$ interval; 5, 5\% interval; PRECIPfull, mean annual precipitation for $1895-2017 ; 2,2 \%$ interval; $0.1,0.1 \%$ interval; $0.01,0.01 \%$ interval]

\begin{tabular}{|c|c|c|c|c|c|c|c|c|}
\hline \multirow{2}{*}{$\begin{array}{l}\text { West (region 2) } \\
\text { flow statistic }\end{array}$} & \multirow[b]{2}{*}{ Regression model } & \multirow[b]{2}{*}{ Regression type } & \multicolumn{6}{|c|}{ Performance metrics } \\
\hline & & & Classification & SE (\%) & RMSE & Pseudo- $R^{2}$ & $\begin{array}{l}\text { Number } \\
\text { censored }\end{array}$ & $\begin{array}{c}\text { Number of } \\
\text { streamgages }\end{array}$ \\
\hline 7Q10_July* & $\begin{array}{l}-1.84+0.09 \ln (C O N T D A) \\
\quad+5.32 \ln (B F I)+2.42 \ln (S L O P E)\end{array}$ & Left censored & NA & 4,426 & 2.75 & 0.6636 & 42 & 68 \\
\hline 30Q10_July & $\begin{array}{l}-0.11+0.31 \ln (C O N T D A) \\
\quad+2.65 \ln (B F I)\end{array}$ & Left censored & NA & 919.8 & 2.11 & 0.47 & 21 & 70 \\
\hline 1Q10_August_P ${ }_{\text {zero }} *$ & $\begin{array}{c}1-\left(e^{-1.16+0.17 \ln (C O N T D A)+3.48 \ln (B F I)}\right. \\
+2.09 \ln (S L O P E) / 1+e^{-1.16+0.17 \ln } \\
(C O N T D A)+3.48 \ln (B F I)+2.09 \ln (S L O P E))\end{array}$ & Logistic & 66.7 & NA & NA & NA & NA & 73 \\
\hline 7Q10_August_P ${ }_{\text {zero }} *$ & $\begin{array}{c}1-\left(e^{-1.66+0.22 \ln (C O N T D A)+2.78 \ln (B F I)}\right. \\
+2.09 \ln (S L O P E) / 1+e^{-1.66+0.22 \ln } \\
(C O N T D A)+2.78 \ln (B F I)+2.09 \ln (S L O P E))\end{array}$ & Logistic & 71.4 & NA & NA & NA & NA & 73 \\
\hline 30Q10_August_P $\mathrm{zero}^{*}$ & 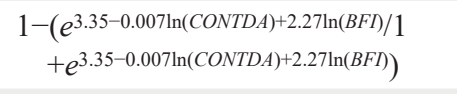 & Logistic & 89.1 & NA & NA & NA & NA & 73 \\
\hline 1Q10_August* & $\begin{array}{l}1.69-0.06 \ln (C O N T D A) \\
\quad+6.16 \ln (B F I)\end{array}$ & Left censored & NA & 13,790 & 3.14 & 0.6879 & 47 & 67 \\
\hline 7Q10_August* & $\begin{array}{l}1.36-0.44 \ln (C O N T D A) \\
\quad+5.55 \ln (B F I)+2.35 \ln (S L O P E)\end{array}$ & Left censored & NA & 2,029 & 2.45 & 0.7487 & 42 & 64 \\
\hline 30Q10_August* & $\begin{array}{l}-4.29+0.56 \ln (C O N T D A) \\
\quad+3.55 \ln (B F I)+1.90 \ln (S L O P E)\end{array}$ & Left censored & NA & 3,318 & 2.65 & 0.5036 & 30 & 68 \\
\hline 1Q10_September_P $\mathrm{z}_{\text {zero }} *$ & 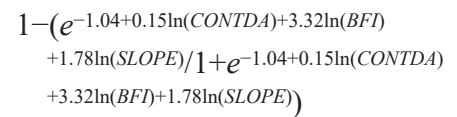 & Logistic & 57.1 & NA & NA & NA & NA & 73 \\
\hline 7Q10_September_P ${ }_{\text {zero }} *$ & $\begin{array}{c}1-\left(e^{-0.06+0.12 \ln (C O N T D A)+3.71 \ln (B F I)}\right. \\
+1.72 \ln (S L O P E) / 1+e^{-0.06+0.12 \ln } \\
(C O N T D A)+3.71 \ln (B F I)+1.72 \ln (S L O P E))\end{array}$ & Logistic & 70.8 & NA & NA & NA & NA & 72 \\
\hline 30Q10_September_P ${ }_{\text {zero }} *$ & $\begin{array}{c}1-\left(e^{-0.19+0.28 \ln (C O N T D A)+3.02 \ln (B F I)}\right. \\
+1.78 \ln (S L O P E) / 1+e^{-0.19+0.28 \ln } \\
(C O N T D A)+3.02 \ln (B F I)+1.78 \ln (S L O P E))\end{array}$ & Logistic & 87.8 & NA & NA & NA & NA & 73 \\
\hline 1Q10_September* & $\begin{array}{l}1.07-0.40 \ln (C O N T D A) \\
\quad+6.32 \ln (B F I)+2.09 \ln (S L O P E)\end{array}$ & Left censored & NA & 3,674 & 2.68 & 0.7431 & 48 & 65 \\
\hline 7Q10_September* & $\begin{array}{l}-1.55-0.01 \ln (C O N T D A) \\
\quad+5.64 \ln (B F I)+2.06 \ln (S L O P E)\end{array}$ & Left censored & NA & 11,780 & 3.09 & 0.6312 & 46 & 65 \\
\hline
\end{tabular}


[classification, percentage of time equation correctly predicts zero flow; SE, standard error; \%, percent; RMSE, root mean square error; pseudo- $R^{2}$, pseudocoefficient of determination; 1Q10, 1-day mean flow with 10-year recurrence interval; $\mathrm{Q}$, flow; Ann, annual; $\mathrm{P}_{\text {zero }}$, probability of zero flow; $e$, base of natural logarithm, approximately equal to 2.7183 ; ln, natural logarithm; CONTDA, contributing drainage area; $B F I$, base-flow index; SLOPE, mean basin slope; NA, not applicable; 7Q10, 7-day mean flow with 10-year recurrence interval; 30Q10, 30-day mean flow with 10-year recurrence interval; KSAT, saturated hydraulic conductivity; FD, flow duration; $99.99,99.99 \%$ interval; $99.90,99.90 \%$ interval; $99,99 \%$ interval; $95,95 \%$ interval; $90,90 \%$ interval; $75,75 \%$ interval; $50,50 \%$ interval; $25,25 \%$ interval; $10,10 \%$ interval; 5, 5\% interval; PRECIPfull, mean annual precipitation for $1895-2017 ; 2,2 \%$ interval; $0.1,0.1 \%$ interval; $0.01,0.01 \%$ interval]

\begin{tabular}{|c|c|c|c|c|c|c|c|c|}
\hline \multirow{2}{*}{$\begin{array}{l}\text { West (region 2) } \\
\text { flow statistic }\end{array}$} & \multirow[b]{2}{*}{ Regression model } & \multirow[b]{2}{*}{ Regression type } & \multicolumn{6}{|c|}{ Performance metrics } \\
\hline & & & Classification & SE (\%) & RMSE & Pseudo- $R^{2}$ & $\begin{array}{l}\text { Number } \\
\text { censored }\end{array}$ & $\begin{array}{c}\text { Number of } \\
\text { streamgages }\end{array}$ \\
\hline 30Q10_September* & $\begin{array}{l}-2.40+0.36 \ln (C O N T D A) \\
\quad+4.20 \ln (B F I)+1.55 \ln (S L O P E)\end{array}$ & Left censored & NA & 1,332 & 2.28 & 0.8139 & 38 & 76 \\
\hline 1Q10_October_P $\mathrm{zero}^{*}$ & $\begin{aligned} 1- & \left(e^{-0.18+0.26 \ln (C O N T D A)+4.25 \ln (B F I)}\right. \\
& +1.56 \ln (S L O P E) / 1+e^{-0.18+0.26 \ln } \\
& (C O N T D A)+4.25 \ln (B F I)+1.56 \ln (S L O P E))\end{aligned}$ & Logistic & 73.1 & NA & NA & NA & NA & 72 \\
\hline 7Q10_October_P $\mathrm{zero} *$ & 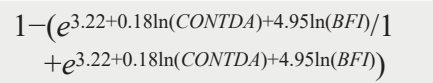 & Logistic & 80 & NA & NA & NA & NA & 73 \\
\hline 30Q10_October_P ${ }_{\text {zero }}{ }^{*}$ & 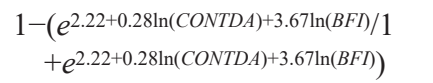 & Logistic & 86.8 & NA & NA & NA & NA & 73 \\
\hline 1Q10_October* & $\begin{array}{l}-0.76+0.07 \ln (C O N T D A) \\
\quad+6.07 \ln (B F I)+1.82 \ln (S L O P E)\end{array}$ & Left censored & NA & 3,555 & 2.67 & 0.7166 & 42 & 65 \\
\hline 7Q10_October* & $\begin{array}{l}-1.82+0.45 \ln (C O N T D A) \\
\quad+6.83 \ln (B F I)+1.56 \ln (S L O P E)\end{array}$ & Left censored & NA & 1,504 & 2.33 & 0.9122 & 45 & 73 \\
\hline 30Q10_October* & $\begin{array}{l}1.20+0.32 \ln (C O N T D A) \\
\quad+5.78 \ln (B F I)\end{array}$ & Left censored & NA & 1,110 & 2.2 & 0.8842 & 38 & 73 \\
\hline 1Q10_November_P ${ }_{\text {zero }} *$ & $\begin{array}{c}1-\left(e^{2.60+0.36 \ln (C O N T D A)+4.94 \ln (B F I) / 1}\right. \\
\left.\quad+e^{2.60+0.36 \ln (C O N T D A)+4.94 \ln (B F I)}\right)\end{array}$ & Logistic & 91.4 & NA & NA & NA & NA & 73 \\
\hline 7Q10_November_P zero ${ }^{*}$ & $\begin{array}{c}1-\left(e^{2.39+0.47 \ln (C O N T D A)+5.36 \ln (B F I) / 1}\right. \\
\left.\quad+e^{2.39+0.47 \ln (C O N T D A)+5.36 \ln (B F I)}\right)\end{array}$ & Logistic & 91.7 & NA & NA & NA & NA & 73 \\
\hline 30Q10_November_P ${ }_{\text {zero }} *$ & $\begin{array}{c}1-\left(e^{1.99-0.44 \ln (C O N T D A)+4.16 \ln (B F I) / 1}\right. \\
\left.\quad+e^{1.99-0.44 \ln (C O N T D A)+4.16 \ln (B F I)}\right)\end{array}$ & Logistic & 87.8 & NA & NA & NA & NA & 73 \\
\hline 1Q10_November & $\begin{array}{l}0.05+0.56 \ln (C O N T D A) \\
\quad+6.76 \ln (B F I)\end{array}$ & Left censored & NA & 1,430 & 2.31 & 0.9059 & 42 & 73 \\
\hline 7Q10_November & $\begin{array}{l}0.09+0.64 \ln (C O N T D A) \\
\quad+6.83 \ln (B F I)\end{array}$ & Left censored & NA & 1,042 & 2.17 & 0.9183 & 39 & 73 \\
\hline 30Q10_November & $\begin{array}{l}0.59+0.59 \ln (C O N T D A) \\
\quad+6.35 \ln (B F I)\end{array}$ & Left censored & NA & 992.2 & 2.14 & 0.9084 & 35 & 73 \\
\hline 1Q10_December_P ${ }_{\text {zero }} *$ & $\begin{array}{c}1-\left(e^{3.42+0.34 \ln (C O N T D A)+5.54 \ln (B F I) / 1}\right. \\
\left.\quad+e^{3.42+0.34 \ln (C O N T D A)+5.54 \ln (B F I)}\right)\end{array}$ & Logistic & 91.7 & NA & NA & NA & NA & 73 \\
\hline 7Q10_December_P ${ }_{\text {zero }} *$ & $\begin{array}{c}1-\left(e^{3.55+0.28 \ln (C O N T D A)+4.85 \ln (B F I) / 1}\right. \\
\left.\quad+e^{3.55+0.28 \ln (C O N T D A)+4.85 \ln (B F I)}\right)\end{array}$ & Logistic & 89.7 & NA & NA & NA & NA & 73 \\
\hline
\end{tabular}


Table 10. Regression equations for estimating selected low-flow frequency statistics for unregulated streams in the west region of Kansas.-Continued

[classification, percentage of time equation correctly predicts zero flow; SE, standard error; \%, percent; RMSE, root mean square error; pseudo- $R^{2}$, pseudocoefficient of determination; $1 \mathrm{Q} 10,1$-day mean flow with 10-year recurrence interval; $\mathrm{Q}$, flow; Ann, annual; $\mathrm{P}_{\text {zero }}$, probability of zero flow; $e$, base of natural logarithm, approximately equal to $2.7183 ;$ ln, natural logarithm; CONTDA, contributing drainage area; $B F I$, base-flow index; SLOPE, mean basin slope; NA, not applicable; 7Q10, 7-day mean flow with 10-year recurrence interval; 30Q10, 30-day mean flow with 10-year recurrence interval; KSAT, saturated hydraulic conductivity; FD, flow duration; $99.99,99.99 \%$ interval; $99.90,99.90 \%$ interval; $99,99 \%$ interval; $95,95 \%$ interval; $90,90 \%$ interval; $75,75 \%$ interval; $50,50 \%$ interval; $25,25 \%$ interval; $10,10 \%$ interval; 5, 5\% interval; PRECIPfull, mean annual precipitation for $1895-2017 ; 2,2 \%$ interval; $0.1,0.1 \%$ interval; $0.01,0.01 \%$ interval]

\begin{tabular}{|c|c|c|c|c|c|c|c|c|}
\hline \multirow{2}{*}{$\begin{array}{l}\text { West (region 2) } \\
\text { flow statistic }\end{array}$} & \multirow[b]{2}{*}{ Regression model } & \multirow[b]{2}{*}{ Regression type } & \multicolumn{6}{|c|}{ Performance metrics } \\
\hline & & & Classification & SE (\%) & RMSE & Pseudo- $R^{2}$ & $\begin{array}{l}\text { Number } \\
\text { censored }\end{array}$ & $\begin{array}{c}\text { Number of } \\
\text { streamgages }\end{array}$ \\
\hline 30Q10_December_P $\mathrm{z}_{\text {zero }}$ * & $\begin{array}{c}1-\left(e^{5.15+0.11 \ln (C O N T D A)+4.52 \ln (B F I) / 1}\right. \\
\left.+e^{5.15+0.11 \ln (C O N T D A)+4.52 \ln (B F I)}\right)\end{array}$ & Logistic & 91.1 & NA & NA & NA & NA & 73 \\
\hline 1Q10_December & $\begin{array}{l}0.70+0.50 \ln (C O N T D A) \\
\quad+6.50 \ln (B F I)\end{array}$ & Left censored & NA & 826.2 & 2.06 & 0.9177 & 39 & 73 \\
\hline 7Q10_December & $\begin{array}{l}0.93+0.52 \ln (C O N T D A) \\
\quad+6.66 \ln (B F I)\end{array}$ & Left censored & NA & 927.9 & 2.11 & 0.9174 & 38 & 73 \\
\hline 30Q10_December & $\begin{array}{l}0.69+0.60 \ln (C O N T D A) \\
\quad+6.13 \ln (B F I)\end{array}$ & Left censored & NA & 873.4 & 2.09 & 0.9075 & 32 & 73 \\
\hline FD_99.99_P $\mathrm{P}_{\text {zero }}{ }^{*}$ & 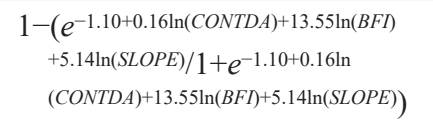 & Logistic & 71.4 & NA & NA & NA & NA & 67 \\
\hline FD_99.90_P $\mathrm{P}_{\text {zero }} *$ & $\begin{array}{c}1-\left(e^{1.72-0.51 \ln (C O N T D A)+6.67 \ln (B F I)}\right. \\
+4.00 \ln (S L O P E) / 1+e^{1.72-0.51 \ln } \\
(C O N T D A)+6.67 \ln (B F I)+4.00 \ln (S L O P E))\end{array}$ & Logistic & 69.2 & NA & NA & NA & NA & 71 \\
\hline FD_99_P ${ }_{\text {zero }} *$ & 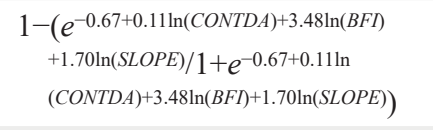 & Logistic & 60.0 & NA & NA & NA & NA & 73 \\
\hline FD_95_P $\mathrm{zero}^{*}$ & $\begin{array}{c}1-\left(e^{0.79-0.50 \ln (C O N T D A)+4.69 \ln (B F I) / 1}\right. \\
\left.+e^{0.79-0.50 \ln (C O N T D A)+4.69 \ln (B F I)}\right)\end{array}$ & Logistic & 83.3 & NA & NA & NA & NA & 73 \\
\hline FD_90_P zero & $\begin{array}{c}1-\left(e^{2.82+0.27 \ln (C O N T D A)+4.08 \ln (B F I) / 1}\right. \\
\left.+\quad e^{2.82+0.27 \ln (C O N T D A)+4.08 \ln (B F I)}\right)\end{array}$ & Logistic & 87.2 & NA & NA & NA & NA & 73 \\
\hline FD_75_P $\mathrm{P}_{\text {zero }} *$ & $\begin{array}{c}1-\left(e^{2.88+0.64 \ln (C O N T D A)+4.79 \ln (B F I) / 1}\right. \\
\left.+e^{2.88+0.64 \ln (C O N T D A)+4.79 \ln (B F I)}\right)\end{array}$ & Logistic & 94.1 & NA & NA & NA & NA & 73 \\
\hline FD_99.99* & $\begin{array}{l}2.55+0.41 \ln (C O N T D A) \\
\quad+20.29 \ln (B F I)\end{array}$ & Left censored & NA & 171,500 & 3.86 & 0.9385 & 62 & 70 \\
\hline FD_99.90* & $\begin{array}{l}2.68+0.07 \ln (C O N T D A) \\
\quad+11.40 \ln (B F I)\end{array}$ & Left censored & NA & 5,140 & 2.81 & 0.901 & 57 & 70 \\
\hline FD_99* & $\begin{array}{l}2.37-0.59 \ln (C O N T D A) \\
\quad+6.65 \ln (B F I)+2.21 \ln (S L O P E)\end{array}$ & Left censored & NA & 8,111 & 2.97 & 0.7357 & 46 & 64 \\
\hline FD_95* & $\begin{array}{l}0.65-0.04 \ln (C O N T D A) \\
\quad+6.74 \ln (B F I)+1.92 \ln (S L O P E)\end{array}$ & Left censored & NA & 1,254 & 2.25 & 0.8193 & 38 & 64 \\
\hline
\end{tabular}


[classification, percentage of time equation correctly predicts zero flow; SE, standard error; \%, percent; RMSE, root mean square error; pseudo- $R^{2}$, pseudocoefficient of determination; 1Q10, 1-day mean flow with 10-year recurrence interval; $\mathrm{Q}$, flow; Ann, annual; $\mathrm{P}_{\text {zero }}$, probability of zero flow; $e$, base of natural logarithm, approximately equal to 2.7183 ; ln, natural logarithm; CONTDA, contributing drainage area; $B F I$, base-flow index; SLOPE, mean basin slope; NA, not applicable; 7Q10, 7-day mean flow with 10-year recurrence interval; 30Q10, 30-day mean flow with 10-year recurrence interval; KSAT, saturated hydraulic conductivity; FD, flow duration; $99.99,99.99 \%$ interval; $99.90,99.90 \%$ interval; $99,99 \%$ interval; $95,95 \%$ interval; $90,90 \%$ interval; $75,75 \%$ interval; $50,50 \%$ interval; $25,25 \%$ interval; $10,10 \%$ interval; 5, 5\% interval; PRECIPfull, mean annual precipitation for $1895-2017 ; 2,2 \%$ interval; $0.1,0.1 \%$ interval; $0.01,0.01 \%$ interval]

\begin{tabular}{|c|c|c|c|c|c|c|c|c|}
\hline \multirow{2}{*}{$\begin{array}{l}\text { West (region 2) } \\
\text { flow statistic }\end{array}$} & \multirow[b]{2}{*}{ Regression model } & \multirow[b]{2}{*}{ Regression type } & \multicolumn{6}{|c|}{ Performance metrics } \\
\hline & & & Classification & SE (\%) & RMSE & Pseudo- $R^{2}$ & $\begin{array}{l}\text { Number } \\
\text { censored }\end{array}$ & $\begin{array}{c}\text { Number of } \\
\text { streamgages }\end{array}$ \\
\hline FD_90* & $\begin{array}{l}2.27+0.16 \ln (C O N T D A) \\
\quad+5.59 \ln (B F I)\end{array}$ & Left censored & NA & 998.1 & 2.15 & 0.7892 & 35 & 70 \\
\hline FD_75 & $\begin{array}{l}0.68+0.57 \ln (C O N T D A) \\
\quad+4.77 \ln (B F I)\end{array}$ & Left censored & NA & 460.2 & 1.76 & 0.8945 & 25 & 73 \\
\hline FD_50 & $\begin{array}{l}-1.89+0.68 \ln (C O N T D A) \\
\quad+2.62 \ln (B F I)+0.56 \ln (K S A T)\end{array}$ & Left censored & NA & 175 & 1.18 & 0.8765 & 12 & 73 \\
\hline FD_25 & $\begin{array}{l}-0.07+0.68 \ln (C O N T D A) \\
\quad+1.94 \ln (B F I)\end{array}$ & Left censored & NA & 120.6 & 0.95 & 0.7641 & 5 & 68 \\
\hline FD_10 & $\begin{array}{l}-0.03+0.68 \ln (C O N T D A) \\
\quad+1.07 \ln (B F I)\end{array}$ & Left censored & NA & 116.5 & 0.93 & 0.6207 & 1 & 70 \\
\hline FD_5 & $\begin{array}{c}-22.44+0.87 \ln (\text { CONTDA }) \\
+6.69 \ln (\text { PRECIPfull })\end{array}$ & Left censored & NA & 88.72 & 0.76 & 0.743 & 0 & 71 \\
\hline FD_2 & $\begin{array}{c}-15.98+0.83 \ln (\text { CONTDA }) \\
+5.22 \ln (\text { PRECIPfull })\end{array}$ & Left censored & NA & 64.59 & 0.59 & 0.8033 & 0 & 71 \\
\hline FD_0.1 & $\begin{array}{l}-6.96+0.65 \ln (\text { CONTDA }) \\
\quad+3.25 \ln (\text { PRECIPfull })\end{array}$ & Left censored & NA & 62.01 & 0.57 & 0.716 & 0 & 71 \\
\hline FD_0.01 & $\begin{array}{l}-3.53+0.57 \ln (\text { CONTDA }) \\
\quad+2.61 \ln (\text { PRECIPfull })\end{array}$ & Left censored & NA & 74.42 & 0.66 & 0.5878 & 0 & 71 \\
\hline Mean_Ann_Q & $\begin{array}{c}-14.59+0.65 \ln (\text { CONTDA }) \\
+4.34 \ln (\text { PRECIPfull })\end{array}$ & Left censored & NA & 86.34 & 0.69 & 0.5324 & 0 & 71 \\
\hline
\end{tabular}

*CONTDA was not statistically significant but was included in the model. 
frequency statistic or flow-duration exceedance interval is estimated to be zero, and the corresponding left-censored regression equation does not need to be used (Eash and Barnes, 2017). If the resulting probability is less than 0.5 , the matching left-censored regression equation should be used to estimate the value for the low-flow frequency statistic or flow-duration exceedance interval.

\section{Accuracy and Limitations of Regression Equations}

The accuracy of the logistic regression equations is described in the classification column; classification being the percentage that the equation correctly predicts zero flow and flow. The logistic regression equations in the east region have a classification range from 56.4 to 97.1 percent, and the mean is 84.1 percent. The classification range of the west region is from 57.1 to 98.1 percent, and the mean is 83.6 percent. The $\mathrm{SE}$ and the root mean square error (RMSE) for the regression equations are listed in tables 9 and 10 . The SE and the RMSE have fairly large ranges in tables 9 and 10, and the values measured in the west region of the State (table 10) are greater than the values measured in the east region of the State (table 9). The smallest SE and RMSE values occur in the late spring (March-May) and early summer (June-August) months, whereas the greatest values occur during the midsummer and early fall (September-November) months. The reason for the large error values in some of the regression equations is the large percentage of censored values used to create the equations, which are in tables 9 and 10. Flows of $0.1 \mathrm{ft}^{3} / \mathrm{s}$ and less are not uncommon in Kansas, especially in the summer and fall months.

The following list describes limitations for using the final regional regression equations developed in this report.

1. Using the equations on locations where the independent variables are outside the ranges of those in this study may generate errors that are greater than the errors listed in tables 9 and 10 . The ranges of the independent variables are listed in table 6 of this report.

2. The regional regression equations in this report are not suitable for streams where flow is substantially affected by regulation, diversion, or urbanization.

3. The basin characteristic computations at ungaged locations should be calculated using the same GIS and other datasets and calculation approaches used in this study. References for basin characteristics are in table 6 and appendix 3, tables 3.1 and 3.2.

4. Special consideration is warranted for censored values and the number of significant figures used. Because of the ability of streamgages to provide continuous data at streamflows of less than $0.1 \mathrm{ft}^{3} / \mathrm{s}$, the censoring threshold used to develop the left-censored regression equations in this report was set at $0.1 \mathrm{ft}^{3} / \mathrm{s}$; therefore, any regression estimates of less than $0.1 \mathrm{ft}^{3} / \mathrm{s}$ should be reported as "less than $0.1 \mathrm{ft}^{3} / \mathrm{s}$."

\section{Example Calculations}

Approaches for applying the regional regression equations listed in tables 9 and 10 are detailed in the following examples.

Example 1.-This example is a calculation of the April 7Q10 (April 7-day low flow with a recurrence interval of 10 years [7Q10_April]) for a streamgage in the east region. Shown in figure 1 is the location of USGS streamgage Stranger Creek near Tonganoxie, Kans. (06892000), as map number 115 . This drainage basin is located entirely within the east region. Estimating the selected low-flow frequency statistic is a one-step process using the equation in table 9. Using the StreamStats web-based GIS tool (https://streamstats .usgs.gov/ss/) or the "StreamStats Gage page" link, CONTDA is measured as $406 \mathrm{mi}^{2}$ and BFI is measured as 0.172 (dimensionless). Because both basin characteristic values are within the range provided in table 6 , the left-censored regression equation is applicable for estimating the April 7Q10. The April 7Q10 left-censored regression equation is as follows:

$$
\begin{gathered}
\text { 7Q10_April }=-0.80+0.81 \ln (C O N T D A)+1.38 \ln (B F I), \\
\text { 7Q10_April=-0.80+0.81 } \ln (406)+1.38 \ln (0.172), \\
\text { 7Q10_April }=-0.80+4.8651-2.4292, \\
\text { 7Q10_April }=1.64 \mathrm{ft}^{3} / \mathrm{s},
\end{gathered}
$$

where

CONTDA is contributing drainage area and

$B F I \quad$ is base-flow index.

Example 2.-This example is a calculation of the 95-percent flow-duration exceedance interval (streamflow that is equaled or exceeded 95 percent of the time) for a streamgage in the west region. Shown in figure 1 is the location of USGS streamgage Smoky Hill River at Elkader, Kans. (06860000), as map number 58. This drainage basin is located entirely within the west region. Estimating the selected lowflow frequency statistic is a one- or two-step process. Step 1 is to estimate the probability of zero flow using the logistic regression equation listed in table 10. Using the StreamStats web-based GIS tool or the "StreamStats Gage page" link, CONTDA is measured as 3,555 $\mathrm{mi}^{2}$ and BFI is measured as 0.202 (dimensionless). Because both basin characteristic values are within the range provided in table 6 , the logistic 
regression and left-censored regression equations are applicable for estimating the 95-percent flow-duration exceedance interval. The 95-percent flow-duration exceedance interval (FD_95_ $\mathrm{P}_{\text {zero }}$ ) logistic regression equation is as follows:

$$
\begin{aligned}
& \text { FD_95_P } \mathrm{zero}=1-\left[\left(e^{0.79+0.50 \ln (C O N T D A)+4.69 \ln (B F T)}\right)\right. \\
& \left./\left(1-\left(e^{0.79+0.50 \ln (C O N T D A)+4.69 \ln (B F I)}\right)\right)\right] \text {, } \\
& \text { FD_95_P } \text { zero }_{1}=1-\left[\left(e^{\left.0.79 \cdot 3,555^{0.50} \cdot 0.202^{4.69}\right)}\right.\right.
\end{aligned}
$$

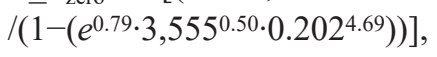

$$
\begin{aligned}
& \text { FD_95_P } \mathrm{zero}=1-\left[\left(7.25 \times 10^{-2}\right) /\left(1-\left(7.25 \times 10^{-2}\right)\right)\right] \text {, } \\
& \text { FD_95_P } \mathrm{P}_{\text {zero }}=1-\left[\left(7.25 \times 10^{-2}\right) /\left(9.28 \times 10^{-1}\right)\right] \text {, } \\
& \text { FD_95_P } \mathrm{zero}_{\text {ero }}=0.92 \text {, }
\end{aligned}
$$

where

$$
\begin{aligned}
& e \quad \text { is the base of natural logarithm, } \\
& \text { approximately equal to } 2.7183 \text {. }
\end{aligned}
$$

Because the estimate for the $\mathrm{FD} \_95$ _ $\mathrm{P}_{\text {zero }}$ is greater than the probability threshold of 0.5 used for this study, the estimate for the FD_95_ $\mathrm{P}_{\text {zero }}$ is zero flow, making step 2 unnecessary. If step 2 were necessary, the FD_95 equation in table 10 would be applied in the same fashion as example 1 .

Example 3.-This example is a calculation of the mean annual flow for a streamgage in the east region. Shown in figure 1 is the location of USGS streamgage Turkey Creek near Seneca, Kans. (06814000), as map number 7. This drainage basin is located entirely within the east region. Estimating the selected low-flow frequency statistic is a one-step process using the left-censored regression equation in table 9. Using the StreamStats web-based GIS tool or the "StreamStats Gage page" link, CONTDA is measured as $276 \mathrm{mi}^{2}$. Using table 3.1, in appendix 3, PRECIPfull is measured as $32.2 \mathrm{in}$. Because all the basin characteristic values are within the range provided in table 6 , the left-censored regression equation is applicable for estimating the mean annual flow. The mean annual flow (Mean_Ann_Q) left-censored regression equation is as follows:

$$
\begin{aligned}
& \text { Mean_Ann_Q }=-14.2+0.82 \ln (\text { CONTDA }) \\
& +4.08 \ln \text { (PRECIPfull), } \\
& \text { Mean_Ann_Q }=-14.2+0.82 \ln (276)+4.08 \ln (32.2) \text {, } \\
& \text { Mean_Ann_Q=-14.2+4.6087+14.1656, }
\end{aligned}
$$

$$
\text { Mean_Ann_Q }=4.57 \mathrm{ft}^{3} / \mathrm{s} \text {, }
$$

where

PRECIPfull is the mean annual precipitation for

1895-2017.

\section{Drainage-Area Ratio Method}

Another method to estimate selected low-flow statistics for an ungaged location is the DAR method. The DAR method assumes that streamflow at an ungaged location is the same per unit area as that for a streamgage upstream or downstream from the ungaged location (Southard, 2013; Eash and Barnes, 2017). Low-flow frequency statistics computed for the streamgage are multiplied by the DAR of the ungaged location to estimate low-flow frequency statistics at the ungaged location. This method is appropriate for streams with a streamgage and is not dependent on how many streamgages are on the same stream (Southard, 2013). "The accuracy of the DAR method depends on similarities in drainage area and other basin characteristics (such as soils, geology, precipitation) between the two locations" (Eash and Barnes, 2017, p. 37). The DAR method usually is applied when the ungaged location is on the same stream as a streamgage and the DAR of the two locations is between 0.5 and 1.5 (Hortness, 2006; Eash and Barnes, 2017). Other studies have examined DARs to verify the range for which the DAR method offers estimates of low-flow statistics that are higher quality than estimates determined using regional regression equations (Eash and Barnes, 2017). Koltun and Schwartz (1987) recommended a DAR range from 0.85 to 1.15 for estimating low-flow statistics in Ohio, whereas Ries and Friesz (2000) concluded that a range from 0.3 to 1.5 was appropriate for low-flow statistics in Massachusetts (Eash and Barnes, 2017). The studies mentioned previously recommend using regression equations for ungaged locations outside of their published DAR ranges. The following equation is the DAR method computation:

$$
Q_{D A R u}=\left(D A_{u} / D A_{g}\right) Q_{o g}
$$

where

$$
\begin{gathered}
Q_{D A R u} \quad \begin{array}{c}
\text { is the low-flow frequency estimate of the } \\
\text { ungaged location, }
\end{array} \\
D A_{u} \quad \begin{array}{c}
\text { is the drainage area of the ungaged location, } \\
D A_{g}
\end{array} \quad \begin{array}{c}
\text { is the drainage area of the streamgage on the } \\
\text { same stream, and }
\end{array} \\
Q_{o g} \quad \begin{array}{l}
\text { is the computed low-flow frequency estimate } \\
\text { from the observed streamgage record. }
\end{array}
\end{gathered}
$$




\section{Comparison to Regression Model}

To evaluate which method, DAR or regional regression equations, is most applicable for Kansas and to determine what range is appropriate for either method, 20 pairs of streamgages were selected for testing estimates of the 7Q10 statistic following a process described by Ries and Friesz (2000) and Eash and Barnes (2017). A set of 26 streamgages composed the 20 pairs, which are listed in table 11. Each pair of streamgages is on the same stream. Of the streams, $8 \mathrm{had}$ only 2 streamgages that could be used for the comparison, 2 had 3 streamgages for comparison, and 1 had 4 streamgages available for comparison. More streamgage pairs are desirable for this analysis, but streamgages with only partial records of flow not affected by regulation, diversion, or urbanization were omitted from this analysis because these streamgages could introduce bias into the results. Two streamgages had observed 7Q10 values of zero; these were changed to less than $0.1 \mathrm{ft}^{3} / \mathrm{s}$ so the mathematical computations would be valid and a comparison could be made. The period of record used to compute the 7Q10 statistic for each streamgage ranged from 11 to 107 years. Drainage area size ranged from 218 to $4,777 \mathrm{mi}^{2}$. Two pairs of streamgages are in the west region of the study area, whereas the other 18 pairs are in the east region.

Using the DAR method and the regional regression equations, 20 pairs of the 7Q10 statistic were calculated. In each pair, it was assumed that one of the locations was ungaged, whereas the other was used as a streamgage. The absolute difference, in percent, was calculated from the observed 7Q10 value and the estimations computed from the DAR method and the regional regression equations for each streamgage that was assumed to be an ungaged location. The results of the absolute differences were then split into four groups; these groups were as follows:

1. DAR estimates with DARs of less than 0.5 and greater than 1.5 ,

2. DAR estimates with DARs between 0.5 and 1.5,

3. regional regression estimates with DARs of less than 0.5 and greater than 1.5 , and

4. regional regression estimates with DARs between 0.5 and 1.5 .

Medians and standard deviations of the absolute differences, in percent, are provided in table 12, and the medians and standard deviations for all the DAR and regional regression equation estimates.

As listed in table 12, the median absolute differences, in percent, for the DAR methods are much lower than the median absolute differences for the regional regression equations, and the standard deviations for the DAR method are much greater than the standard deviations for the regional regression equations. The Wilcoxon signed-rank test was completed using $\mathrm{R}$ statistical software to determine the statistical difference between the medians of the groups in this analysis (R Core Team, 2020). The results indicated that the DAR method estimates are not significantly different from the regional regression equations. The $p$-value equaled 0.44 when the DAR was less than 0.5 and greater than 1.5. The $p$-value equaled 0.56 when the DAR was between 0.5 and 1.5. To summarize, based on the Wilcoxon signed-rank test, either the DAR method or the regional regression equations can be used to estimate flow statistics for ungaged locations in Kansas. Other studies have published guidelines indicating what DARs are acceptable before the use of regression equations is preferred. Several studies have reported that the DAR is ideal when the ratio is between 0.5 and 1.5 (Ries and Friesz, 2000). Many of the studies that published this guideline did not offer any scientific basis for how it was set (Ries and Friesz, 2000). This analysis concludes the DAR method or regional regression equations can be used to estimate flow statistics in Kansas based on the results of the Wilcoxon signed-rank test. It should be noted that the median absolute difference, in percent, is significantly lower for the DAR method when the ratios are between 0.5 and 1.5 .

\section{Example Calculation}

Example 4.-This example is a calculation of a DAR for the annual 7-day mean low flow with a recurrence interval of 5 years (7Q5) statistic for a stream location in the east region. The USGS streamgage Stranger Creek near Potter, Kans. (06891810), is shown in figure 1 as map number 115 . This streamgage was assumed to be an ungaged location for this example. Another streamgage, Stranger Creek near Tonganoxie, Kans. (06892000), also is shown in figure 1 as map number 116. This streamgage will be used as the streamgage in this example. This drainage basin is located entirely within the east region of the study area. Using the StreamStats web-based GIS tool or the "StreamStats Gage page" link, CONTDA is measured as $184 \mathrm{mi}^{2}$ for the ungaged location and $406 \mathrm{mi}^{2}$ for the streamgage. Listed in appendix table 2.1 is the computed 7Q5 statistic for the streamgage as $0.30 \mathrm{ft}^{3} / \mathrm{s}$. The DAR method equation for the 7Q5 low-flow frequency statistic is as follows:

$$
\begin{gathered}
Q_{D A R u}=\left(D A_{u} / D A_{g}\right) Q_{o g}, \\
Q_{D A R u}=\left(184 \mathrm{mi}^{2} / 406 \mathrm{mi}^{2}\right) 0.30 \mathrm{ft}^{3} / \mathrm{s}, \\
Q_{D A R u}=(0.45) 0.30 \mathrm{ft}^{3} / \mathrm{s}, \\
Q_{D A R u}=0.14 \mathrm{ft}^{3} / \mathrm{s} .
\end{gathered}
$$


Table 11. Pairs of U.S. Geological Survey streamgages for drainage-area ratio versus regional regression equations analysis.

[USGS, U.S. Geological Survey; CONTDA, contributing drainage area; DAR, drainage-area ratio; 7Q10, minimum 7-day annual low flow with a 10-year recurrence interval; \%, percent; NE, Nebraska; MO, Missouri; C, Creek; NR, near; KS, Kansas; R, River; OK, Oklahoma]

\begin{tabular}{|c|c|c|c|c|c|c|c|c|c|c|}
\hline \multirow{2}{*}{$\begin{array}{l}\text { Pair } \\
\text { number }\end{array}$} & \multirow[b]{2}{*}{ Map number } & \multirow{2}{*}{$\begin{array}{l}\text { USGS station } \\
\text { number }\end{array}$} & \multirow[b]{2}{*}{ USGS station name } & \multirow[b]{2}{*}{ CONTDA } & \multirow[b]{2}{*}{ DAR } & \multirow{2}{*}{$\begin{array}{c}\text { Observed } \\
7010\end{array}$} & \multicolumn{2}{|c|}{ DAR method } & \multicolumn{2}{|c|}{ Regional regression equations } \\
\hline & & & & & & & Estimate & $\begin{array}{c}\text { Absolute difference } \\
(\%)\end{array}$ & Estimate & $\begin{array}{c}\text { Absolute difference } \\
(\%)\end{array}$ \\
\hline \multirow[t]{2}{*}{1} & 4 & 06810500 & $\begin{array}{l}\text { LITTLE NEMAHA } \\
\text { RIVER NEAR SYRA- } \\
\text { CUSE, NE }\end{array}$ & 218 & 0.28 & 0.41 & 3.11 & 660.7 & 0.10 & 75.6 \\
\hline & 5 & 06811500 & $\begin{array}{l}\text { LITTLE NEMAHA } \\
\text { RIVER AT AUBURN, } \\
\text { NE }\end{array}$ & 792 & 3.63 & 11.3 & 1.49 & 86.9 & 1.37 & 87.8 \\
\hline \multirow[t]{2}{*}{2} & 11 & 06817500 & $\begin{array}{l}\text { NODAWAY RIVER } \\
\text { NEAR BURLINGTON } \\
\text { JUNCTION, MO }\end{array}$ & 1,240 & 0.82 & 6.91 & 24.7 & 257.8 & 0.53 & 92.3 \\
\hline & 12 & 06817700 & $\begin{array}{l}\text { NODAWAY RIVER } \\
\text { NEAR GRAHAM, } \\
\text { MO }\end{array}$ & 1,520 & 1.23 & 30.3 & 8.47 & 72.0 & 2.30 & 92.4 \\
\hline \multirow[t]{2}{*}{3} & 15 & 06820500 & $\begin{array}{l}\text { PLATTE RIVER NEAR } \\
\text { AGENCY, MO }\end{array}$ & 1,760 & 0.74 & 3.39 & 16.7 & 393.1 & 0.10 & 97.1 \\
\hline & 18 & 06821190 & $\begin{array}{l}\text { PLATTE RIVER AT } \\
\text { SHARPS STATION, } \\
\text { MO }\end{array}$ & 2,371 & 1.35 & 22.5 & 4.57 & 79.7 & 1.75 & 92.2 \\
\hline \multirow[t]{2}{*}{4} & 36 & 06845000 & $\begin{array}{l}\text { SAPPA C NR OBERLIN, } \\
\text { KS* }\end{array}$ & 923 & 0.62 & 0.10 & 0.01 & 87.6 & 0.10 & 0.0 \\
\hline & 37 & 06845110 & SAPPA C NR LYLE, KS & 1,488 & 1.61 & 0.02 & 0.16 & 706.1 & 0.10 & 400.0 \\
\hline \multirow[t]{2}{*}{5} & 86 & 06881000 & $\begin{array}{l}\text { BIG BLUE RIVER } \\
\text { NEAR CRETE, NE }\end{array}$ & 2,716 & 0.62 & 26.3 & 26.9 & 2.2 & 2.61 & 90.1 \\
\hline & 90 & 06882000 & $\begin{array}{l}\text { BIG BLUE RIVER AT } \\
\text { BARNESTON, NE }\end{array}$ & 4,370 & 1.61 & 43.3 & 42.3 & 2.1 & 2.10 & 95.1 \\
\hline \multirow[t]{2}{*}{6} & 86 & 06881000 & $\begin{array}{l}\text { BIG BLUE RIVER } \\
\text { NEAR CRETE, NE }\end{array}$ & 2,716 & 0.57 & 26.3 & 29.6 & 12.4 & 2.61 & 90.1 \\
\hline & 92 & 06882510 & $\begin{array}{l}\text { BIG BLUE R AT } \\
\text { MARYSVILLE, KS }\end{array}$ & 4,777 & 1.76 & 52.0 & 46.3 & 11.0 & 2.96 & 94.3 \\
\hline \multirow[t]{2}{*}{7} & 90 & 06882000 & $\begin{array}{l}\text { BIG BLUE RIVER AT } \\
\text { BARNESTON, NE }\end{array}$ & 4,370 & 0.91 & 43.3 & 47.6 & 10.0 & 2.10 & 95.1 \\
\hline & 92 & 06882510 & $\begin{array}{l}\text { BIG BLUE R AT } \\
\text { MARYSVILLE, KS }\end{array}$ & 4,777 & 1.09 & 52.0 & 47.3 & 9.1 & 2.96 & 94.3 \\
\hline
\end{tabular}


Table 11. Pairs of U.S. Geological Survey streamgages for drainage-area ratio versus regional regression equations analysis.-Continued

[USGS, U.S. Geological Survey; CONTDA, contributing drainage area; DAR, drainage-area ratio; 7Q10, minimum 7-day annual low flow with a 10-year recurrence interval; \%, percent; NE, Nebraska; MO, Missouri; C, Creek; NR, near; KS, Kansas; R, River; OK, Oklahoma]

\begin{tabular}{|c|c|c|c|c|c|c|c|c|c|c|}
\hline \multirow{2}{*}{$\begin{array}{l}\text { Pair } \\
\text { number }\end{array}$} & \multirow[b]{2}{*}{ Map number } & \multirow{2}{*}{$\begin{array}{l}\text { USGS station } \\
\text { number }\end{array}$} & \multirow[b]{2}{*}{ USGS station name } & \multirow[b]{2}{*}{ CONTDA } & \multirow[b]{2}{*}{ DAR } & \multirow{2}{*}{$\begin{array}{c}\text { Observed } \\
7010\end{array}$} & \multicolumn{2}{|c|}{ DAR method } & \multicolumn{2}{|c|}{ Regional regression equations } \\
\hline & & & & & & & Estimate & $\begin{array}{c}\text { Absolute difference } \\
(\%)\end{array}$ & Estimate & $\begin{array}{c}\text { Absolute difference } \\
(\%)\end{array}$ \\
\hline \multirow[t]{2}{*}{8} & 87 & 06881200 & $\begin{array}{l}\text { TURKEY CREEK } \\
\text { NEAR WILBER, NE }\end{array}$ & 461 & 0.64 & 0.19 & 1.27 & 580.7 & 0.10 & 46.5 \\
\hline & 88 & 06881380 & $\begin{array}{l}\text { TURKEY CREEK } \\
\text { NEAR DEWITT, NE }\end{array}$ & 724 & 1.57 & 2.00 & 0.29 & 85.3 & 0.10 & 95.0 \\
\hline \multirow[t]{2}{*}{9} & 93 & 06883000 & $\begin{array}{l}\text { LITTLE BLUE RIVER } \\
\text { NEAR DEWEESE, NE }\end{array}$ & 984 & 0.42 & 8.09 & 16.6 & 105.5 & 1.97 & 75.6 \\
\hline & 95 & 06884000 & $\begin{array}{l}\text { LITTLE BLUE RIVER } \\
\text { NEAR FAIRBURY, } \\
\text { NE }\end{array}$ & 2,350 & 2.39 & 39.7 & 19.3 & 51.3 & 2.16 & 94.6 \\
\hline \multirow[t]{2}{*}{10} & 93 & 06883000 & $\begin{array}{l}\text { LITTLE BLUE RIVER } \\
\text { NEAR DEWEESE, NE }\end{array}$ & 984 & 0.36 & 8.09 & 14.8 & 83.6 & 1.97 & 75.6 \\
\hline & 96 & 06884025 & $\begin{array}{l}\text { LITTLE BLUE RIVER } \\
\text { AT HOLLENBERG, } \\
\text { KS }\end{array}$ & 2,752 & 2.80 & 41.5 & 22.6 & 45.5 & 2.03 & 95.1 \\
\hline \multirow[t]{2}{*}{11} & 93 & 06883000 & $\begin{array}{l}\text { LITTLE BLUE RIVER } \\
\text { NEAR DEWEESE, NE }\end{array}$ & 984 & 0.29 & 8.09 & 12.8 & 57.9 & 1.97 & 75.6 \\
\hline & 98 & 06884400 & $\begin{array}{l}\text { LITTLE BLUE R NR } \\
\text { BARNES, KS }\end{array}$ & 3,351 & 3.41 & 43.5 & 27.5 & 36.7 & 2.09 & 95.2 \\
\hline \multirow[t]{2}{*}{12} & 95 & 06884000 & $\begin{array}{l}\text { LITTLE BLUE RIVER } \\
\text { NEAR FAIRBURY, } \\
\text { NE }\end{array}$ & 2,350 & 0.85 & 39.7 & 35.5 & 10.6 & 2.16 & 94.6 \\
\hline & 96 & 06884025 & $\begin{array}{l}\text { LITTLE BLUE RIVER } \\
\text { AT HOLLENBERG, } \\
\text { KS }\end{array}$ & 2,752 & 1.17 & 41.5 & 46.5 & 11.9 & 2.03 & 95.1 \\
\hline \multirow[t]{2}{*}{13} & 95 & 06884000 & $\begin{array}{l}\text { LITTLE BLUE RIVER } \\
\text { NEAR FAIRBURY, } \\
\text { NE }\end{array}$ & 2,350 & 0.70 & 39.7 & 30.5 & 23.2 & 2.16 & 94.6 \\
\hline & 98 & 06884400 & $\begin{array}{l}\text { LITTLE BLUE R NR } \\
\text { BARNES, KS }\end{array}$ & 3,351 & 1.43 & 43.5 & 56.6 & 30.2 & 2.09 & 95.2 \\
\hline \multirow[t]{2}{*}{14} & 96 & 06884025 & $\begin{array}{l}\text { LITTLE BLUE RIVER } \\
\text { AT HOLLENBERG, } \\
\text { KS }\end{array}$ & 2,752 & 0.82 & 41.5 & 35.7 & 14.0 & 2.03 & 95.1 \\
\hline & 98 & 06884400 & $\begin{array}{l}\text { LITTLE BLUE R NR } \\
\text { BARNES, KS }\end{array}$ & 3,351 & 1.22 & 43.5 & 50.6 & 16.3 & 2.09 & 95.2 \\
\hline
\end{tabular}


Table 11. Pairs of U.S. Geological Survey streamgages for drainage-area ratio versus regional regression equations analysis.-Continued

[USGS, U.S. Geological Survey; CONTDA, contributing drainage area; DAR, drainage-area ratio; 7Q10, minimum 7-day annual low flow with a 10-year recurrence interval; \%, percent; NE, Nebraska; MO, Missouri; C, Creek; NR, near; KS, Kansas; R, River; OK, Oklahoma]

\begin{tabular}{|c|c|c|c|c|c|c|c|c|c|c|}
\hline \multirow{2}{*}{$\begin{array}{l}\text { Pair } \\
\text { number }\end{array}$} & \multirow[b]{2}{*}{ Map number } & \multirow{2}{*}{$\begin{array}{l}\text { USGS station } \\
\text { number }\end{array}$} & \multirow[b]{2}{*}{ USGS station name } & \multirow[b]{2}{*}{ CONTDA } & \multirow[b]{2}{*}{ DAR } & \multirow{2}{*}{$\begin{array}{l}\text { Observed } \\
7010\end{array}$} & \multicolumn{2}{|c|}{ DAR method } & \multicolumn{2}{|c|}{ Regional regression equations } \\
\hline & & & & & & & Estimate & $\begin{array}{c}\text { Absolute difference } \\
(\%)\end{array}$ & Estimate & $\begin{array}{c}\text { Absolute difference } \\
(\%)\end{array}$ \\
\hline \multirow[t]{2}{*}{15} & 110 & 06890100 & $\begin{array}{l}\text { DELAWARE R NR } \\
\text { MUSCOTAH, KS }\end{array}$ & 431 & 0.47 & 0.21 & 0.43 & 108.5 & 0.10 & 51.5 \\
\hline & 111 & 06890500 & $\begin{array}{l}\text { DELAWARE R AT VAL- } \\
\text { LEY FALLS, KS }\end{array}$ & 922 & 2.14 & 0.92 & 0.44 & 52.0 & 0.10 & 89.1 \\
\hline \multirow[t]{2}{*}{16} & 152 & 06917500 & $\begin{array}{r}\text { MARMATON R NR } \\
\text { FORT SCOTT, KS }\end{array}$ & 388 & 0.85 & 0.17 & 0.32 & 91.6 & 0.10 & 39.8 \\
\hline & 153 & 06917560 & $\begin{array}{l}\text { MARMATON RIVER } \\
\text { NEAR RICHARDS, } \\
\text { MO }\end{array}$ & 455 & 1.17 & 0.37 & 0.19 & 47.8 & 0.10 & 73.2 \\
\hline \multirow[t]{2}{*}{17} & 152 & 06917500 & $\begin{array}{r}\text { MARMATON R NR } \\
\text { FORT SCOTT, KS }\end{array}$ & 388 & 0.36 & 0.17 & 0.39 & 132.9 & 0.10 & 39.8 \\
\hline & 154 & 06918060 & $\begin{array}{l}\text { MARMATON RIVER } \\
\text { NEAR NEVADA, MO }\end{array}$ & 1,074 & 2.77 & 1.07 & 0.46 & 57.1 & 0.10 & 90.7 \\
\hline \multirow[t]{2}{*}{18} & 153 & 06917560 & $\begin{array}{l}\text { MARMATON RIVER } \\
\text { NEAR RICHARDS, } \\
\text { MO }\end{array}$ & 455 & 0.42 & 0.37 & 0.45 & 21.5 & 0.10 & 73.2 \\
\hline & 154 & 06918060 & $\begin{array}{l}\text { MARMATON RIVER } \\
\text { NEAR NEVADA, MO }\end{array}$ & 1,074 & 2.36 & 1.07 & 0.88 & 17.7 & 0.10 & 90.7 \\
\hline \multirow[t]{2}{*}{19} & 196 & 07155590 & $\begin{array}{l}\text { CIMARRON R NR } \\
\text { ELKHART, KS* }\end{array}$ & 2,416 & 0.56 & 0.10 & 3.33 & $3,233.6$ & 0.10 & 0.0 \\
\hline & 198 & 07157000 & $\begin{array}{l}\text { CIMARRON RIVER } \\
\text { NEAR MOCANE, OK }\end{array}$ & 4,305 & 1.78 & 5.94 & 0.18 & 97.0 & 0.10 & 98.3 \\
\hline \multirow[t]{2}{*}{20} & 236 & 07185700 & $\begin{array}{l}\text { SPRING RIVER AT LA } \\
\text { RUSSELL, MO }\end{array}$ & 306 & 0.72 & 26.8 & 21.3 & 20.5 & 1.52 & 94.3 \\
\hline & 237 & 07185765 & $\begin{array}{l}\text { SPRING RIVER AT } \\
\text { CARTHAGE, MO }\end{array}$ & 425 & 1.39 & 29.6 & 37.3 & 25.8 & 1.52 & 94.9 \\
\hline
\end{tabular}

*Reported values are zero, used 0.1 cubic foot per second for analysis. 
Table 12. Medians and standard deviations of the absolute differences between annual mean 7-day low flow with a recurrence interval of 10 years (7010) using observed streamflow, the drainage-area ratio method, and regional regression equations.

$[<$, less than; $>$, greater than $]$

\begin{tabular}{lcccc}
\hline \multicolumn{1}{c}{ Group } & $\begin{array}{c}\text { Drainage-area } \\
\text { ratio range }\end{array}$ & $\begin{array}{c}\text { Number in } \\
\text { group }\end{array}$ & $\begin{array}{c}\text { Median absolute difference } \\
\text { (percent) }\end{array}$ & $\begin{array}{c}\text { Standard } \\
\text { deviation }\end{array}$ \\
\hline All estimates & All & 80 & 87.2 & 373.1 \\
Drainage-area ratio method & All & 40 & 51.7 & 523.4 \\
& $<0.5$ and $>1.5$ & 19 & 57.9 & 199.3 \\
& 0.5 to 1.5 & 21 & 25.8 & 701.5 \\
Regional regression equations & All & 40 & 92.4 & 56.1 \\
& $<0.5$ and $>1.5$ & 19 & 90.7 & 74.4 \\
& 0.5 to 1.5 & 21 & 94.3 & 30.6 \\
\hline
\end{tabular}

\section{StreamStats Implementation}

StreamStats is a USGS web-based GIS tool that permits users to find streamflow statistics, basin characteristics, and other information for user-selected locations on streams. "The StreamStats home page (https://streamstats.usgs.gov/ss/) provides links to a more detailed description of the program, a user's manual, descriptions of the outputs, definitions of basin characteristics and streamflow statistics, limitations for use of the application, and other information" (U.S. Geological Survey, 2021). It is recommended that users read the user's manual before trying to use the application.

"StreamStats makes the process of computing streamflow statistics for ungaged locations much faster, more accurate, and more consistent than previously used manual methods" (Eash and Barnes, 2017, p. 43). Another advantage of StreamStats is that it makes streamflow statistics for streamgages readily available and more easily accessed (Eash and Barnes, 2017). Streamflow statistics can be needed at any location alongside a stream and can aid with water-resources planning and management; the design of facilities such as wastewatertreatment plants; and the design of structures such as roads, bridges, culverts, and levees (Eash and Barnes, 2017). Also, planners, regulators, and others often need to recognize the physical and climatic characteristics (basin characteristics) of the drainage basins upstream from places of interest to support them in understanding the processes that affect water availability and quality at these locations (Eash and Barnes, 2017).

The regression equations provided in this report will be implemented in the USGS StreamStats web-based GIS tool. StreamStats will provide users the ability to estimate selected low-flow frequency and flow-duration statistics for ungaged streams in Kansas.

\section{Summary}

Knowledge of the magnitude, frequency, and duration of low flows is critical for water-supply management; reservoir design; waste-load allocation; and the preservation of water quality and quantity for irrigation, recreation, and ecological conservation purposes. Because of the importance of low-flow statistics, the U.S. Geological Survey (USGS), in cooperation with the Kansas Water Office, began a statewide study in 2018 to develop regression equations to estimate selected low-flow frequency and flow-duration statistics for ungaged stream locations in Kansas.

Major components of this study included (1) streamgage selection; (2) computation of selected low-flow statistics at 254 continuous-record streamgages within Kansas and the surrounding States with at least 10 years of record through September 30, 2017; (3) measurement of 13 basin characteristics for each streamgage within the study area; (4) development of 167 regression equations to estimate selected statistics at ungaged stream locations based on basin characteristics; (5) explanation of the drainage-area ratio (DAR) method and instances when it is appropriate to be used instead of regression equations; and (6) incorporation of the regression equations published in this report into the USGS StreamStats application.

A Mann-Kendall test was completed on each streamgage included in the regression analysis for this study because trends in the $n$-day data can introduce bias in the results of the low-flow frequency analysis. Of the streamgages tested, 77 indicated a significant trend for the 7-day annual minimum flow. An upward trend was seen at 56 streamgages, whereas a decreasing trend was observed at 21 streamgages. The trend analysis of minimum 1-day mean flows for 17 streamgages, minimum 7-day mean flows for 14 streamgages, and minimum 30-day mean flows for 7 streamgages produced tau and probability values that may be difficult to interpret because of the presence of several zero flows. When reviewing streamgage records over periods of a few years to a few decades, trends commonly appear; however, when these records are reviewed 
in the context of decades to centuries, short-term trends can be recognized as being part of much longer term fluctuations. No streamgage was omitted from this study because of the presence of a positive or negative trend in its dataset.

Methods described in the report for estimating selected flow-duration and low-flow frequency statistics are applicable to streams in Kansas that are not substantially affected by diversion, regulation, or urbanization by using regression equations. Regression equations were developed to estimate the annual and monthly 1-, 7-, and 30-day mean low flows with a recurrence interval of 10 years at ungaged stream locations. Regression equations were developed to estimate the flow-duration of 0.01-, 0.1-, 2-, 5-, 10-, 25-, 50-, 75-, 90-, 95-, 99-, 99.9-, and 99.99-percent exceedance intervals. Regression equations were developed for the mean annual flows as well.

For this study, Kansas was split into an east and west region and different regression equations were developed for each region. Logistic and left-censored regression techniques were used in this report because of the presence of several zero flows in the record periods. These techniques are recommended when zero flows are present and significant in a dataset. The threshold for censored data was set at 0.1 cubic foot per second $\left(\mathrm{ft}^{3} / \mathrm{s}\right)$ because of the ability of a streamgage to provide continuous data during extreme low-flow conditions. A variety of performance metrics is provided to estimate the accuracy of each equation. These equations are only appropriate for streams in Kansas that are not substantially affected by diversion, regulation, or urbanization. Basin characteristics of these ungaged locations also need to be within the range of the basin characteristics used to develop these equations. The large amount of zero flows in some datasets are believed to be the cause of large errors in some of the previously published equations. Any equation that yields a result of less than $0.1 \mathrm{ft}^{3} / \mathrm{s}$ should be reported as "less than $0.1 \mathrm{ft}^{3} / \mathrm{s}$."

The DAR method was described, and instances when it is appropriate to be used instead of regression equations were presented. Previous studies indicated that it is preferable to have a ratio of drainage areas that is between 0.5 and 1.5 for the DAR method although no scientific basis for this guideline has been provided. The results of the Wilcoxon signed-rank test for this analysis of Kansas indicated that the absolute differences, in percent, were not significantly different when comparing the DAR method to the regional regression equations, regardless of what the DAR was. The DAR method had the smallest median absolute difference, in percent, when the ratio was between 0.5 and 1.5. Therefore, the results of this study indicate the DAR method is appropriate for use in Kansas when estimating streamflow at an ungaged location if there is a nearby streamgage on the same river or stream where the DAR is between 0.5 and 1.5 and the low-flow statistic at the nearby streamgage is not zero.
The regional regression equations developed in this study are not intended for use at ungaged stream locations where the basin characteristics are outside the ranges of those used to develop the equations published in this study. Geographicinformation-system software is required to measure the basin characteristics included as independent variables in the regression equations. All the regression equations developed for this study will be incorporated into the USGS StreamStats web-based geographic-information-system tool. StreamStats will provide users with a set of annual and monthly low-flow frequency statistics, as well as flow-duration and mean annual flow estimates for ungaged stream locations within Kansas. StreamStats also will provide users with the basin characteristics for the locations.

\section{Acknowledgments}

The author gratefully acknowledges the following U.S. Geological Survey personnel: Julie Kiang and the SWToolbox help team, for their assistance in using SWToolbox; Anthony Gotvald, for his assistance with using the R program swmrQW for the regression analysis; Kyle Puls, for his assistance in writing R scripts for this study; Chantelle Davis, for her assistance with streamgage selection used for the regression analysis; and Brian Klager and Talia Klein, for their assistance with computing basin characteristics and creating maps to be used in figures of this report. The author also would like to express deep appreciation to the many other U.S. Geological Survey personnel who helped collect and analyze stage and streamflow data used in this report.

\section{References Cited}

Barbie, D.L., Wehmeyer, L.L., and May, J.E., 2012, Analysis of trends in selected streamflow statistics for the Concho River Basin, Texas, 1916-2009: U.S. Geological Survey Scientific Investigations Report 2012-5193, 15 p. [Also available at https://doi.org/10.3133/sir20125193.]

Barlow, P.M., Cunningham, W.L., Zhai, T., and Gray, M., 2015, U.S. Geological Survey Groundwater Toolbox, a graphical and mapping interface for analysis of hydrologic data (ver. 1.0) - User guide for estimation of base flow, runoff, and groundwater recharge from streamflow data: U.S. Geological Survey Techniques and Methods, book 3, chap. B10, 27 p., accessed January 2019 at https://doi.org/ 10.3133/tm3B10. 
Barnes, H.H., Jr., 1979, Programs and plans-Low-flow programs: U.S. Geological Survey Surface Water Branch Technical Memorandum no. 79.06, accessed January 2019 at https://water.usgs.gov/admin/memo/SW/sw79.06.html.

Eash, D.A., and Barnes, K.K., 2017, Methods for estimating selected low-flow frequency statistics and harmonic mean flows for streams in Iowa (ver. 1.1, November 2017): U.S. Geological Survey Scientific Investigations Report 2012-5171, 99 p., accessed December 2018 at https://doi.org/10.3133/sir20125171.

England, J.F., Jr., Cohn, T.A., Faber, B.A., Stedinger, J.R., Thomas, W.O., Jr., Veilleux, A.G., Kiang, J.E., and Mason, R.R., Jr., 2019, Guidelines for determining flood flow frequency_Bulletin 17C (ver. 1.1, May 2019): U.S. Geological Survey Techniques and Methods, book 4, chap. B5, 148 p. [Also available at https://doi.org/ 10.3133/tm4B5.]

Fry, J.A., Xian, G.S., Jin, S., Dewitz, J., Homer, C., Yang, L., Barnes, C.A., Herold, N., and Wickham, J.D., 2011, Completion of the 2006 National Land Cover Database for the conterminous United States: Photogrammetric Engineering and Remote Sensing, v. 77, no. 9, p. 858-864.

Funkhouser, J.E., Eng, K., and Moix, M.W., 2008, Low-flow characteristics and regionalization of low-flow characteristics for selected streams in Arkansas: U.S. Geological Survey Scientific Investigations Report 2008-5065, 161 p., accessed February 2019 at https://doi.org/10.3133/ sir20085065.

Furness, L.W., 1959, Kansas streamflow characteristicsPart 1-Flow duration: Topeka, Kansas, Kansas Water Resources Board Technical Report no. 1, 213 p.

Gebert, W.A., Graczyk, D.J., and Krug, W.R., 1987, Average annual runoff in the United States, 1951-80: U.S. Geological Survey Hydrologic Atlas 710, scale 1:7,500,000. [Also available at https://doi.org/10.3133/ha710.]

Goodin, D.G., Mitchell, J.E., Knapp, M.C., and Bivens, R.E., 2004, Climate and weather atlas of Kansas: Lawrence, Kansas, Kansas Geological Survey Educational Series 12, accessed September 15, 2020, at http:/www.kgs.ku.edu/ Publications/Bulletins/ED12/KGS_ED12.pdf.

Gotvald, A.J., 2016, Selected low-flow frequency statistics for continuous-record streamgages in Georgia, 2013: U.S. Geological Survey Scientific Investigations Report 2016-5037, 20 p., accessed January 2019 at https://doi.org/ $10.3133 / \operatorname{sir} 20165037$.
Gotvald, A.J., 2017, Methods for estimating selected low-flow frequency statistics and mean annual flow for ungagged locations on streams in North Georgia: U.S. Geological Survey Scientific Investigations Report 2017-5001, 25 p., accessed January 2019 at https://doi.org/10.3133/ $\operatorname{sir} 20175001$.

Helsel, D.R., Hirsch, R.M., Ryberg, K.R., Archfield, S.A., and Gilroy, E.J., 2020, Statistical methods in water resources: U.S. Geological Survey Techniques and Methods, book 4, chap. A3, 458 p. [Also available at https://doi.org/ $10.3133 / \mathrm{tm} 4 \mathrm{a} 3$.

Hortness, J.E., 2006, Estimating low-flow frequency statistics for unregulated streams in Idaho: U.S. Geological Survey Scientific Investigations Report 2006-5035, 31 p. [Also available at https://doi.org/10.3133/sir20065035.]

Iman, R.L., and Conover, W.J., 1983, A modern approach to statistics: New York, John Wiley and Sons, Inc., 497 p.

Jordan, P.R., 1983, Kansas streamflow characteristicsMagnitude and frequency of low flows of unregulated streams in Kansas, and estimation of flow-duration curves for ungaged sites: Kansas Water Office Technical Report No. 17,55 p.

Juracek, K.E., 2015, Streamflow characteristics and trends at selected streamgages in southwest and south-central Kansas: U.S. Geological Survey Scientific Investigations Report 2015-5167, 20 p., accessed February 2019 at https://doi.org/10.3133/sir20155167.

Juracek, K.E., and Eng, K., 2017, Streamflow alteration at selected sites in Kansas: U.S. Geological Survey Scientific Investigations Report 2017-5046, 75 p., accessed January 2019 at https://doi.org/10.3133/sir20175046.

Juracek, K.E., Eng, K., Carlisle, D.M., and Wolock, D.M., 2017, Streamflow alteration and habitat ramifications for a threatened fish species in the central United States: River Research and Applications, v. 33, no. 7, p. 993-1003. [Also available at https://doi.org/10.1002/rra.3148.]

Kendall, M.G., 1938, A new measure of rank correlation: Biometrika, v. 30, no. 1-2, p. 81-93, accessed July 14, 2015, at https://doi.org/10.1093/biomet/30.1-2.81.

Kiang, J.E., Flynn, K.M., Zhai, T., Hummel, P., and Granato, G., 2018, SWToolbox-A surface-water tool-box for statistical analysis of streamflow time series: U.S. Geological Survey Techniques and Methods, book 4, chap. A11, 33 p., accessed August 2020 at https://doi.org/10.3133/tm4A11. 
Koltun, G.F., and Schwartz, R.R., 1987, Multiple-regression equations for estimating low flows at ungaged stream sites in Ohio: U.S. Geological Survey Water-Resources Investigations Report 86-4354, 39 p. plus 6 pl. [Also available at https://doi.org/10.3133/wri864354.]

Kroll, C., Luz, J., Allen, B., and Vogel, R.M., 2004, Developing a watershed characteristics database to improve low streamflow prediction: Journal of Hydrologic Engineering, v. 9, no. 2, p. 116-125, accessed April 11, 2011, at https://doi.org/10.1061/(ASCE)10840699(2004)9:2(116).

Lins, H.F., Hirsch, R.M., and Kiang, J., 2010, Water-The Nation's fundamental climate issue - A white paper on the U.S. Geological Survey role and capabilities: U.S. Geological Survey Circular 1347, 9 p. [Also available at https://doi.org/10.3133/cir1347.]

Lorenz, D.L., 2014, smwrQW-R functions to support water-quality data analysis for statistical methods in water resources: U.S. Geological Survey R Archive Network, accessed August 5, 2020, at https://github.com/USGSR/smwrQW.

Martin, G.R., and Arihood, L.D., 2010, Methods for estimating selected low-flow frequency statistics for unregulated streams in Kentucky: U.S. Geological Survey Scientific Investigations Report 2010-5217, 83 p. plus 2 pl. [Also available at https://doi.org/10.3133/sir20105217.]

Moody, D.W., Chase, E.B., and Aronson, D.A., comps., 1986, National water summary 1985-Hydrologic events and surface-water resources: U.S. Geological Survey WaterSupply Paper 2300, 506 p. [Also available at https://doi.org/ 10.3133/wsp2300.]

National Oceanic and Atmospheric Administration, National Centers for Environmental Information, 2020, Climate at a glance: National Oceanic and Atmospheric Administration digital data, accessed February 27, 2020, at https://www.ncdc.noaa.gov/cag/.

Painter, C.C., Heimann, D.C., and Lanning-Rush, J.L., 2017, Methods for estimating annual exceedanceprobability streamflows for streams in Kansas based on data through water year 2015 (ver. 1.1, September 2017): U.S. Geological Survey Scientific Investigations Report 2017-5063, 20 p., accessed January 2020 at https://doi.org/ $10.3133 / \operatorname{sir} 20175063$.
Perry, C.A., Wolock, D.M., and Artman, J.C., 2004a, Estimates of flow duration, mean flow, and peak-discharge frequency values for Kansas stream locations: U.S. Geological Survey Scientific Investigations Report 2004-5033, 651 p. [Also available at https://doi.org/10.3133/sir20045033.]

Perry, C.A., Wolock, D.M., and Artman, J.C., 2004b, Estimates of median flows for streams on the 1999 Kansas Surface Water Register: U.S. Geological Survey Scientific Investigations Report 2004-5032, 219 p. [Also available at https://doi.org/10.3133/sir20045032.]

PRISM Climate Group, 2020, Normal annual precipitation grid for the conterminous United States: PRISM Climate Group web page, accessed April 15, 2020, at https://www.prism.oregonstate.edu/explorer/.

R Core Team, 2020, R-A language and environment for statistical computing: R Foundation for Statistical Computing, Vienna, Austria, accessed January 2020 at https://www.Rproject.org/.

Rasmussen, P.P., and Perry, C.A., 2000, Estimation of peak streamflows for unregulated rural streams in Kansas: U.S. Geological Survey Water-Resources Investigations Report 2000-4079, 33 p. [Also available at https://doi.org/10.3133/ wri004079.]

Ries, K.G., III, 2006, Selected streamflow statistics for streamgaging stations in northeastern Maryland, 2006: U.S. Geological Survey Open-File Report 2006-1335, 16 p. [Also available at https://doi.org/10.3133/ofr20061335.]

Ries, K.G., III, and Friesz, P.J., 2000, Methods for estimating low-flow statistics for Massachusetts streams: U.S. Geological Survey Water-Resources Investigations Report 2000-4135, 81 p. [Also available at https://doi.org/10.3133/ wri004135.]

Ries, K.G., III, Newson J.K., Smith, M.J., Guthrie, J.D., Steeves, P.A., Haluska, T.L., Kolb, K.R., Thompson, R.F., Santoro, R.D., and Vraga, H.W., 2017, StreamStats, version 4: U.S. Geological Survey Fact 2017-3046, 4 p. [Also available at https://doi.org/10.3133/fs20173046.] [Supersedes USGS Fact Sheet 2008-3067.]

Riggs, H.C., 1972, Low-flow investigations: U.S. Geological Survey Techniques of Water-Resources Investigations, book 4, chap. B1, 18 p. [Also available at https://doi.org/10.3133/ twri04B1.]

Runkel, R.L., Crawford, C.G., and Cohn, T.A., 2004, Load estimator (LOADEST) - A FORTRAN program for estimating constituent loads in streams and rivers: U.S. Geological Survey Techniques and Methods, book 4, chap. A5, 69 p. [Also available at https://doi.org/10.3133/tm4A5.] 
Searcy, J.K., 1959, Flow-duration curves, Manual of hydrology-Part 2-Low-flow techniques: U.S. Geological Survey Water-Supply Paper 1542-A, 33 p. [Also available at https://doi.org/10.3133/wsp1542A.]

Southard, R.E., 2013, Computed statistics at streamgages, and methods for estimating low-flow frequency statistics and development of regional regression equations for estimating low-flow frequency statistics at ungaged locations in Missouri: U.S. Geological Survey Scientific Investigations Report 2013-5090, 28 p. [Also available at https://doi.org/ $10.3133 / \operatorname{sir} 20135090$.

Studley, S.E., 2000, Estimated flow-duration curves for selected ungaged sites in the Cimarron and lower Arkansas River Basins in Kansas: U.S. Geological Survey WaterResources Investigations Report 2000-4113, 43 p. [Also available at https://doi.org/10.3133/wri004113.]

Studley, S.E., 2001, Estimated flow-duration curves for selected ungaged sites in Kansas: U.S. Geological Survey Water-Resources Investigations Report 2001-4142, 90 p. [Also available at https://doi.org/10.3133/wri014142.]

U.S. Geological Survey, 2006, National Land Cover Database: U.S. Geological Survey web page, accessed February 24, 2020, at https:/www.usgs.gov/centers/eros/science/ national-land-cover-database?qt-science_center_objects= 0\#qt-science_center_objects.

U.S. Geological Survey, 2010, USGS EROS archive-Digital elevation-Global Multi-resolution Terrain Elevation Data 2010 (GMTED2010): U.S. Geological Survey web page, accessed February 24, 2020, at https://doi.org/10.5066/ F7J38R2N.

U.S. Geological Survey, 2018, USGS water data for the Nation: U.S. Geological Survey National Water Information System database, accessed June 1, 2018, at https://doi.org/ 10.5066/F7P55KJN.
U.S. Geological Survey, 2019, USGS EROS archive-Digital elevation-Shuttle Radar Topography Mission (SRTM) 1 arc-second global: U.S. Geological Survey web page, accessed February 24, 2020, at https://doi.org/10.5066/ F7PR7TFT.

U.S. Geological Survey, 2020, WaterWatch: U.S. Geological Survey web page, accessed March 30, 2020, at https:// waterwatch.usgs.gov/?id=ww_about.

U.S. Geological Survey, 2021, StreamStats: U.S. Geological Survey digital data, accessed February 23, 2021, at https://streamstats.usgs.gov/ss/.

Wieczorek, M.E., 2014, Area- and depth- weighted averages of selected SSURGO variables for the conterminous United States and District of Columbia: U.S. Geological Survey Data Series, v. 866, accessed May 6, 2020, at https://doi.org/ $10.3133 / \mathrm{ds} 866$.

Williams-Sether, T., and Gross, T.A., 2016, Regression equations to estimate seasonal flow duration, $n$-day high-flow frequency, and $n$-day low-flow frequency at sites in North Dakota using data through water year 2009: U.S. Geological Survey Scientific Investigations Report 2015-5184, 12 p., accessed January 2019 at https://doi.org/10.3133/ sir20155184.

Ziegeweid, J.R., Lorenz, D.L., Sanocki, C.A., and Czuba, C.R., 2015, Methods for estimating flow-duration curve and low-flow frequency statistics for ungaged locations on small streams in Minnesota: U.S. Geological Survey Scientific Investigations Report 2015-5170, 23 p., accessed January 2019 at https://doi.org/10.3133/sir20155170. 
Appendixes 1-3 


\section{Appendix 1. Flow-Duration Curve Information}

This appendix contains two tables with flow-duration curve information. Flow-duration curve information for the streamgages used in the regression analysis in this report is provided in table 1.1, and flow-duration curve information for all active streamgages in the State of Kansas is provided in table 1.2 (tables 1.1 and 1.2 are available for download at https://doi.org/10.3133/sir20215100). Statistics provided in tables 1.1 and 1.2 were computed using data from the U.S. Geological Survey National Water Information System database (U.S. Geological Survey, 2018).

\section{Reference Cited}

U.S. Geological Survey, 2018, USGS water data for the Nation: U.S. Geological Survey National Water Information System database, accessed June 1, 2018, at https://doi.org/ 10.5066/F7P55KJN. 


\section{Appendix 2. Low-Flow Frequency Statistics Information}

This appendix contains four tables with low-flow frequency statistics information. Annual low-flow frequency statistics for the streamgages used in the regression analysis in this report are provided in table 2.1, monthly low-flow frequency statistics for the streamgages used in the regression analysis in this report are provided in table 2.2, annual low-flow frequency statistics for the active streamgages in the State of Kansas are provided in table 2.3, and the results of the Mann-Kendall test for trends for the streamgages used in the regression analysis in this report are provided in table 2.4 (tables 2.1-2.4 are available for download at https://doi.org/ $10.3133 /$ sir20215100). Statistics provided in tables 2.1-2.4 were computed using data from the U.S. Geological Survey National Water Information System database (U.S. Geological Survey, 2018).

\section{Reference Cited}

U.S. Geological Survey, 2018, USGS water data for the Nation: U.S. Geological Survey National Water Information System database, accessed June 1, 2018, at https://doi.org/ 10.5066/F7P55KJN. 


\section{Appendix 3. Basin Characteristic Information}

This appendix contains two tables with basin characteristic information. The basin characteristics considered for use in the regression analysis in this report for the streamgages within region 1 of the study area (east region) are listed in table 3.1, and the basin characteristics considered for use in the regression analysis in this report for the streamgages located within region 2 (west region) of the study area are listed in table 3.2 (tables 3.1 and 3.2 are available for download at https://doi.org/10.3133/sir20215100). 

For more information about this publication, contact: Director, USGS Kansas Water Science Center

1217 Biltmore Drive

Lawrence, KS 66049

785-842-9909

For additional information, visit: https://www.usgs.gov/centers/kswsc Publishing support provided by the

Rolla Publishing Service Center 

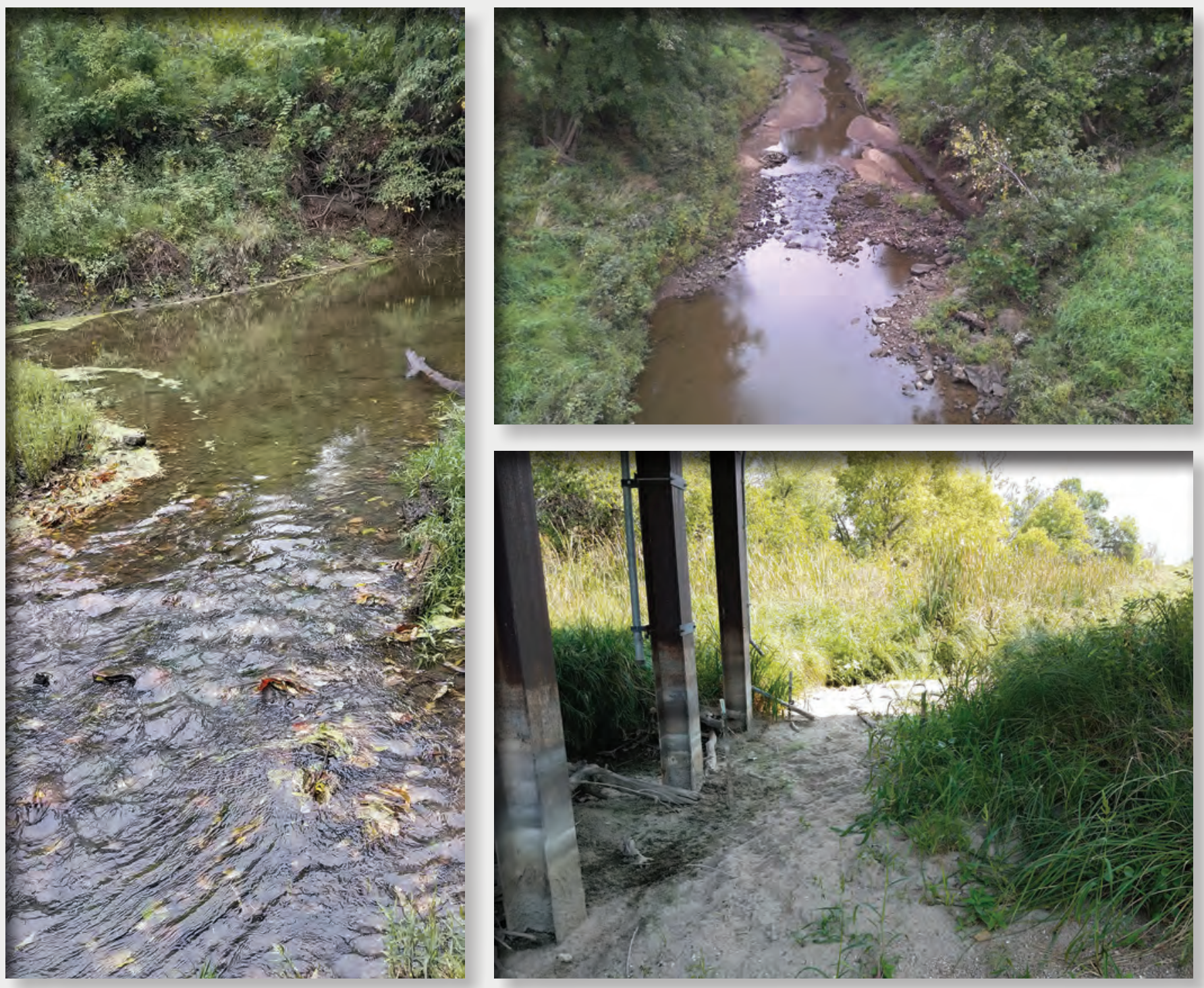

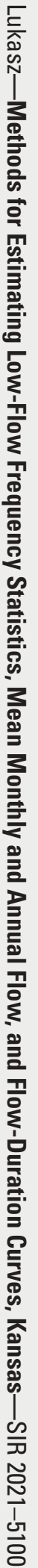

